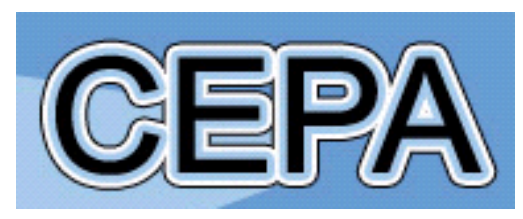

Centre for Efficiency and Productivity Analysis

Working Paper Series

No. WP03/2020

Quadratic-mean-of-order-r

Indexes of Output, Input and

Productivity

Hideyuki Mizobuchi and Valentin Zelenyuk

Date: February 2020

\author{
School of Economics \\ University of Queensland \\ St. Lucia, Qld. 4072 \\ Australia
}

ISSN No. 1932 - 4398 


\title{
Quadratic-mean-of-order-r Indexes of Output, Input and Productivity*
}

\author{
Hideyuki Mizobuchi ${ }^{\dagger}$ and Valentin Zelenyuk ${ }^{*}$
}

February 2020

\begin{abstract}
The use of appropriate index numbers is indispensable for measuring economic phenomena precisely. Various indexes have been proposed in the literature, spanning several centuries. In this paper, we propose the quadratic-mean-of-order- $r$ indexes of output, input and productivity. Each index is a family of indexes that unify many of the existing indexes, including the most popular ones. We also show that all index number formulae belonging to these families are superlative indexes. This is considered as a generalization of the equivalence of Fisher and Malmquist indexes, shown by Diewert (1992). Our results also give new justifications for output and input comparison and productivity measurement via other interesting indexes such as the implicit Walsh index. We also apply the discussed indexes to the US industry production accounts.
\end{abstract}

Keywords: index numbers, superlative index, quadratic-mean-of-order- $r$ index, Fisher index, Malmquist index, implicit Walsh index, time reversal test

JEL classification: C14, D24, E31, O47

\footnotetext{
* This is a substantially revised version of CEPA Working Paper Series No. WP06/2018, where we have refined statements, addressed feedback of colleagues, corrected typos and added an empirical application.

${ }^{\dagger}$ Faculty of Economics, Ryukoku University, 67 Fukakusa Tsukamoto-cho, Fushimi-ku, Kyoto $612-$ 8577, Japan, mizobuchi@econ.ryukoku.ac.jp.

* School of Economics and Centre for Efficiency and Productivity Analysis, The University of Queensland, 530, Colin Clark Building (39), St. Lucia, Brisbane, QLD 4072, Australia; v.zelenyuk@uq.edu.au.
} 


\section{Introduction}

Index numbers play an essential role in the measurement of various economic phenomena. Their importance have increased even more in the light of the vast amounts of data becoming available to researchers. They provide effective tools for handling and summarizing enormous amounts of information including what these days is often referred to as 'big data'.

Studies on index numbers have a long history and include the early classical works of Fisher (1922), Konüs (1924) and Frisch $(1930,1936)$ to mention a few. ${ }^{1}$ In this paper, we contribute to this literature by introducing the quadratic-mean-of-order- $r$ indexes of output, input and productivity and show that each coincides with the corresponding Malmquist index under some mild conditions. ${ }^{2}$ The Malmquist indexes were introduced by Caves et al. (1982), and have been used and popularized in many studies since then (e.g., see Färe et al. (1994), Kumar and Russell (2002), Henderson and Russell (2005), Diewert and Fox (2010)). ${ }^{3}$ On the other hand, the application of the quadratic-mean-oforder- $r$ index has been limited to the cost of living index, as examined by Diewert (1976) and Hill (2006).

Index numbers are widely used to measure the economic variables such as price, quantity and productivity in the general situation of multiple products. There exist two types of index numbers: the theoretical index number (simply, theoretical index) and the empirical index number formula (simply, empirical index). The former is constructed by making use of general aggregator functions such as revenue, cost and distance function. It conceptualizes the measurement of changes in economic variables, based on consumer and producer theory. ${ }^{4}$ On the other hand, the latter is a formula of prices and quantities observed in the two periods. It summarizes the price (quantity) change of different goods by taking into account the information of the corresponding quantities (prices).

A large number of empirical indexes have been proposed and a variety of formulae such as the Laspeyres, Paasche, Fisher and Törnqvist indexes, are still widely used for constructing various official statistics for observing important economic phenomena (inflation, economic growth, etc.) as well as being used to facilitate academic research. There has been an on-going debate regarding the appropriate choice of empirical indexes. Historically, there are several approaches to this problem. ${ }^{5}$ Among them, we focus on two major approaches: economic and axiomatic (sometimes called test) approaches.

The economic approach attempts to find the empirical index that is exactly equal to the theoretical index under two conditions: optimizing behaviour of a household or a firm,

\footnotetext{
${ }^{1}$ See Diewert (1993b) and Balk (2008) for a historic overview.

2 Thus, the quadratic-mean-of-order- $r$ output index coincides with the Malmquist output index, the quadratic-mean-of-order- $r$ input index coincides with the Malmquist input index and the quadraticmean-of-order- $r$ productivity index coincides with the Malmquist productivity index.

${ }^{3}$ Currently, the Malmquist indexes are widely adopted by many empirical applications as well as theoretical studies. See Balk (1998) and Färe et al. (1998), and more recently Diewert and Fox (2010, 2014) and Sickles and Zelenyuk (2019).

${ }^{4}$ For example, the Konüs cost of living index intends to capture inflation by measuring the change in the minimum cost of reaching a given level of utility. See Konüs (1924) and Diewert (1976).

${ }^{5}$ Diewert (1993a, 1993b) summarizes the past studies on this problem into five approaches and calls the economic and the axiomatic approaches 'two major approaches'.
} 
and a certain functional form for the aggregator function. ${ }^{6}$ If, such an empirical index exists, it is called an exact index. ${ }^{7}$ By showing that an empirical index coincides with a theoretical index, one can clarify what it really captures. However, this coincidence between two indexes is valid only when the assumed functional form is an appropriate representation of the underlying preferences or technology. ${ }^{8}$

A superlative index is a special case of exact indexes which coincides with a corresponding theoretical index under the assumption of a flexible functional form for the aggregator function. ${ }^{9}$ Thus, the coincidence between superlative indexes and corresponding theoretical indexes is robust to the specification of underlying preferences and technology. ${ }^{10}$ Therefore, by showing that an empirical index is a superlative index for some theoretical index, one effectively justifies the particular empirical index on the grounds of economic theory. ${ }^{11}$ As a result, the use of superlative indexes is strongly recommended. ${ }^{12}$

So far, it is known that there exist two superlative indexes for output, input and productivity. They are the Törnqvist and the Fisher indexes. Caves et al. (1982) show that the Törnqvist indexes of output, input and productivity are superlative indexes in the case of perfect competition, which Diewert and Fox (2010) later extend to the case of monopolistic competition. On the other hand, Diewert (1992) shows that the Fisher indexes of output, input and productivity are also superlative indexes. ${ }^{13}$ All the above studies show that, under general conditions, the Törnqvist and the Fisher indexes of output, input and productivity coincide with the corresponding Malmquist indexes. ${ }^{14}$ The Malmquist indexes are the representative theoretical indexes for measurement of output and input change and productivity growth, which are defined with the help of distance functions. ${ }^{15}$

The axiomatic approach is another popular approach to the selection of empirical indexes. It proposes a set of axioms that an empirical index should satisfy and evaluates competing empirical indexes on the basis of the axioms that each index satisfies or fails. As Fisher (1922) and more recently Diewert (1992) and the International Labour Office

\footnotetext{
${ }^{6}$ By optimizing behaviour, here we mean the household's utility maximizing or cost minimizing behaviour, and the firm's cost minimizing or revenue maximizing or profit maximizing behaviour.

${ }^{7}$ Konüs and Byushgens (1926) is the first study that showed that various empirical indexes are exact indexes.

${ }^{8}$ Here, the assumption of optimizing behaviour needs to be valid as well. Otherwise, it is necessary to estimate underlying technology or preferences to implement theoretical indexes.

${ }^{9}$ The concept of a 'superlative index' is first introduced by Diewert (1976).

${ }^{10}$ The flexible functional form can approximate the true function to the second order. See Lau (1986) and Chambers (1988), and more recently Serletis and Feng (2015) and Serletis and Isakin (2017).

${ }^{11}$ Barnett and Choi (2008) introduce the more general definition of superlative indexes than Diewert (1976).

${ }^{12}$ See International Labour Office et al. (2004).

${ }^{13}$ Caves et al. (1982) employ the translog functional form and Balk (1998) calls it the Diewert distance function.

${ }^{14}$ The Hicks-Moorsteen productivity index, which is defined as the ratio of the Malmquist output and input indexes, is another important theoretical productivity index. Mizobuchi (2017) also shows that the Törnqvist productivity index is a superlative index for the Hicks-Moorsteen productivity index.

${ }^{15}$ Balk $(1993,1998)$ and Grifell-Tatjé et al. (2016) also explore the relationship between the Fisher and the Malmquist indexes. While their approach has the advantage of assuming weaker regularity conditions on underlying technology, the Fisher and the Malmquist indexes do not necessarily precisely coincide with each other under their specification of technology. Since we are primarily interested in the conditions that theoretical indexes turn out to be exactly equal to empirical indexes, we follow a different approach from these studies.
} 
et al. (2004) advocate, the Fisher index satisfies the largest number of axioms. While the Törnqvist index does not satisfy all the axioms that the Fisher index does, it satisfies several important axioms such as the time reversal test. The Laspeyres and the Paasche indexes, which are still widely used for official statistics, do not satisfy the time-reversal test. Thus, both the Törnqvist and Fisher indexes are often deemed more desirable than the Laspeyres and Paasche indexes under the axiomatic approach.

There also exist other empirical indexes that are justifiable from the axiomatic approach such as the Walsh and implicit Walsh indexes. These two indexes satisfy several important axioms (such as the time reversal test, which the Laspeyres and the Paasche indexes do not satisfy) as well as some monotonicity axioms (such as monotonicity in quantities, which the Törnqvist index does not satisfy). Thus, when we merely apply the existing results in the economic approach, the Walsh and implicit Walsh indexes, which are considered better than the Törnqvist index from the axiomatic approach, are at risk of being discarded. ${ }^{16}$

The main purpose of this paper is to show that from the standpoint of the economic approach, we can justify a much larger number of empirical indexes, which include some empirical indexes that satisfy many desirable axioms yet are so far believed not to be justifiable from the economic approach. In particular, in this paper, we introduce the quadratic-mean-of-order- $r$ indexes of output, input and productivity, which are empirical index number formulae of observed prices and quantities. These indexes generalize and unify many existing indexes, including the most popular ones.

We show that these indexes coincide with the Malmquist indexes of output, input and productivity, provided that a firm engages in optimizing behaviour and the aggregator function has a certain flexible functional form, which we introduce in this paper. ${ }^{17}$ Since there are no restrictions on the parameter $r$ of the index, the quadratic-mean-of-order- $r$ index can be regarded as a family of empirical indexes. Thus, our result is interpreted as showing all indexes that belong to this family are superlative indexes for the Malmquist indexes.

The quadratic-mean-of-order- $r$ index is a family of empirical indexes. Two important special cases of the quadratic-mean-of-order- $r$ index include the implicit Walsh index (for $r=1$ ) and the Fisher index (for $r=2$ ). Therefore, by generalizing the result of Diewert (1992), we explore the possibility of approximating theoretical indexes by employing a variety of empirical indexes including the implicit Walsh indexes, which are known to satisfy many desirable axioms.

The counterpart of our result is found in the literature of the consumer price index. Diewert (1976) shows that the quadratic-mean-of-order- $r$ price (quantity) index is a superlative index for a theoretical price (quantity) index of consumption goods. ${ }^{18}$ While being a fundamental result in itself, it does not guarantee that a similar conclusion holds

\footnotetext{
${ }^{16}$ More precisely, the International Labour Office et al. (2004), which explores the axiomatic properties of the Walsh index rather than the implicit Walsh, concludes that "The remaining two indexes, the Walsh and Törnqvist indexes, both satisfy the time reversal test but the Walsh index emerges as being 'better' since it passes 16 of 20 tests whereas the Törnqvist only satisfies 11 tests." As we show, this argument holds for the implicit Walsh. While it mainly focuses on price index, all the analysis can be translated into quantity index, which includes output and input indexes.

${ }^{17} \mathrm{We}$ introduce the flexible functional form based on the quadratic mean of order $r$ that generalizes a functional form adopted by Diewert (1992).

${ }^{18}$ Theoretical price index of consumption goods is called a cost of living index.
} 
in the context of the indexes of output, input and especially productivity, because three additional complications come into play in those cases. ${ }^{19}$ Indeed, constructing a productivity index is somewhat more complicated than constructing a price (quantity) index for a household. While the latter is simply the aggregation of price (quantity) relatives for consumption goods, the former involves the aggregations of quantity relatives for outputs as well as inputs. Moreover, measuring output (input) changes is also more complicated than constructing price changes for a household. While the latter aggregates price relatives conditioning on a single index of utility, the former aggregates quantity relatives for outputs (inputs) by conditioning on multiple inputs (outputs) and technology. Furthermore, the underlying aggregator functions need to be able to reflect technology change, possibly a non-neutral technology change allowing for greater improvement in the use of some inputs or in producing more of some outputs relative to others. Meanwhile, change in consumer's preferences is rarely considered in the cost of living index. ${ }^{20}$

The quadratic-mean-of-order- $r$ index is a family of empirical indexes. How much do those indexes belonging to the same family differ? In order to answer this question, we apply the quadratic-mean-of-order- $r$ index as well as other well-known indexes to the US industry-level production accounts. For this particular example, while all quadraticmean-of-order- $r$ indexes are almost the same for the range of $r$ from 0.5 to 5 , they could significantly diverge from each other for $r$ much larger than that. This is true for the indexes of output, input and productivity.

The rest of the paper is organized as follows. Section 2 illustrates the model of production and introduces the definitions of various index numbers. Section 3 compares the axiomatic properties of a variety of empirical indexes with special attention to the implicit Walsh indexes. Section 4 introduces a family of functional forms for the output and input distance functions and shows that they are flexible functional forms. Section 5 demonstrates that the quadratic-mean-of-order- $r$ indexes of output, input and productivity are all superlative indexes. Section 6 is dedicated to an empirical application of the quadratic-mean-of-order- $r$ index under different values of $r$ to US industry data. Finally, Section 7 makes concluding remarks.

\section{Two Types of Indexes}

This section introduces a collection of index numbers. They are classified into two types of indexes: theoretical index number and empirical index number formula. Later we explore the exact relationships among several important indexes of these two types.

\subsection{Theoretical Index Numbers}

While the cost of living index, which is a theoretical index for consumer price, is constructed based on consumer theory, theoretical indexes for measuring changes in output, input and productivity are constructed based on production theory. Below we summarize some key results of production theory that are needed for further derivations. $^{21}$

\footnotetext{
${ }^{19}$ For example, according to the result by Diewert (1992), the quadratic-mean-of-order- $r$ price index is applicable to the consumer price index but it is not sure whether the quadratic-mean-of-order- $r$ price index is applicable to other price statistics such as the producer price index and GDP deflator..

${ }^{20}$ Balk (1989) is a rare exception which disentangles the effect of preference changes from the cost of living index.

${ }^{21}$ See Färe and Primont (1995) for more details, which we follow here.
} 
Suppose that a firm produces outputs $y=\left(y_{1}, \ldots, y_{M}\right) \in \mathbb{R}_{+}^{M}$ from inputs $x=$ $\left(x_{1}, \ldots, x_{N}\right) \in \mathbb{R}_{+}^{N}$. The technology set $T^{t}$, which is the set of all feasible combinations of inputs and outputs, characterizes the firm's technology available at period $t$. It is formally defined as:

$$
T^{t} \equiv\left\{(x, y) \in \mathbb{R}_{+}^{N+M}: x \text { can produce } y \text { in period } t\right\}
$$

We assume that the technology satisfies the following regularity conditions: ${ }^{22}$ (T.1) no free lunch: $\left(0_{N}, y\right) \notin T^{t}$ for any $y \neq 0_{M}$; (T.2) inaction is possible with any input: $\left(x, 0_{M}\right) \in T^{t}$ for any $x \geq 0$; (T.3) strong disposability of inputs and outputs: if $(x, y) \in$ $T^{t}$, then $\left(x^{*}, y^{*}\right) \in T^{t}$ for any $x^{*} \geq x$ and any $y^{*} \leq y$; (T.4) $T^{t}$ is closed; (T.5) the output set $P^{t}(x) \equiv\left\{y \in \mathbb{R}_{+}^{M}:(x, y) \in T^{t}\right\}$ is bounded for any $x \in \mathbb{R}_{+}^{N}$; and (T.6) the production function $f^{t}\left(y_{-1}, x\right) \equiv \max _{y_{1}}\left\{y_{1}:\left(x, y_{1}, y_{-1}\right) \in T^{t}\right\}$ is twice continuously differentiable. These conventional axioms on the technology (T.1)-(T.5) guarantee that the distance functions introduced below are well-defined. ${ }^{23}$ The boundary of the technology set $T^{t}$ is called the production frontier of period $t$. The last property (T.6) indicates that the production frontier is smooth enough for the corresponding distance function to be twice continuously differentiable. ${ }^{24}$

The period $t$ technology is alternatively represented by either the output set or the input set, which is the subset of the technology set. They are defined as:

$$
\begin{aligned}
P^{t}(x) & \equiv\left\{y \in \mathbb{R}_{+}^{M}:(x, y) \in T^{t}\right\} \\
L^{t}(y) & \equiv\left\{x \in \mathbb{R}_{+}^{N}:(x, y) \in T^{t}\right\}
\end{aligned}
$$

The boundaries of these sets are referred to as the output isoquant and the input isoquant, respectively, and are defined as:

$$
\begin{gathered}
\text { Isoq } P^{t}(x) \equiv\left\{y \in P^{t}(x): \lambda y \notin P^{t}(x), \forall \lambda \in(1,+\infty)\right\} ; \\
\text { Isoq } L^{t}(y) \equiv\left\{x \in L^{t}(y): \lambda x \notin L^{t}(y), \forall \lambda \in(0,1)\right\} .
\end{gathered}
$$

The period $t$ output distance function $D_{o}^{t}: \mathbb{R}_{+}^{N+M} \rightarrow \mathbb{R}_{+} \cup\{+\infty\}$ characterizes the technology of period $t .{ }^{25}$ It is defined as:

\footnotetext{
${ }^{22}$ Vector notation: $y \geq y^{\prime}$ indicates $y_{m} \geq y_{m}^{\prime}$ for any $m ; y \gg y^{\prime}$ indicates $y_{m}>y_{m}^{\prime}$ for any $m ; y>y^{\prime}$ indicates $y_{m} \geq y_{m}^{\prime}$ for any $m$ and $y \neq y^{\prime} ; 0_{M}$ and $1_{M}$ denotes $M$ dimensional vector of zeros and ones, respectively; and $x_{-1}=\left(x_{2}, \ldots, x_{N}\right)$.

${ }^{23}$ We follow the regularity conditions given by Färe and Primont (1995). The regularity conditions proposed by Diewert and Fox (2010) can also be used.

${ }^{24}$ We can also impose differentiability on the production frontier by adopting the input requirement function rather than the production function. Or we can alternatively impose differentiability on the distance function itself. The distance function does not need to be twice continuously differentiable for defining productivity indexes, but its twice continuous differentiability appears to be indispensable for implementing the exact index number approach.

${ }^{25}$ See Shephard (1970).
} 


$$
D_{o}^{t}(x, y) \equiv \inf \left\{\theta>0: y / \theta \in P^{t}(x)\right\}
$$

It measures the radial distance from outputs $y$ to the output isoquant of period $t$ by the minimum contraction of outputs $y$. Therefore, the following equation is a necessary and sufficient condition for outputs $y$ to be on the output isoquant of $P^{t}(x)$ :

$$
D_{o}^{t}(x, y)=1
$$

By definition, the output distance function is linearly homogeneous in outputs, so that:

$$
\lambda D_{o}^{t}(x, y)=D_{o}^{t}(x, \lambda y), \quad \forall \lambda>0 \text { and } \forall(x, y) \in \mathbb{R}_{+}^{N+M} .
$$

Throughout this paper, we assume that the technology exhibits constant returns to scale. ${ }^{26}$ Under this condition, the output distance function is homogeneous of degree minus one in inputs, so that:

$$
\lambda^{-1} D_{o}^{t}(x, y)=D_{o}^{t}(\lambda x, y), \quad \forall \lambda>0 \text { and } \forall(x, y) \in \mathbb{R}_{+}^{N+M} .
$$

Thus, under constant returns to scale technology, the output distance function is a homogeneous function with respect to outputs as well as inputs. By applying the Euler's theorem on differentiable homogeneous functions, we can derive the next lemma, which play a key role in deriving the exact relationship between theoretical and empirical indexes.

Lemma 1 (Diewert 1976): Suppose that the technology exhibits constant returns to scale and the output distance function $D_{o}$ is twice differentiable at $\left(x^{*}, y^{*}\right)$. Then, $D_{o}$ satisfies the following equations:

$$
\begin{gathered}
-\sum_{n=1}^{N} \frac{\partial D_{o}\left(x^{*}, y^{*}\right)}{\partial x_{n}} x_{n}^{*}=D_{o}\left(x^{*}, y^{*}\right), \\
\sum_{v=1}^{N} \frac{\partial^{2} D_{o}\left(x^{*}, y^{*}\right)}{\partial x_{u} \partial x_{v}} x_{v}^{*}=-2 \frac{\partial D_{o}\left(x^{*}, y^{*}\right)}{\partial x_{u}}, \quad \forall u \in[1, \ldots, N], \\
\sum_{n=1}^{N} \frac{\partial^{2} D_{o}\left(x^{*}, y^{*}\right)}{\partial x_{n} \partial y_{m}} x_{n}^{*}=-\frac{\partial D_{o}\left(x^{*}, y^{*}\right)}{\partial y_{m}}, \quad \forall m \in[1, \ldots, M] . \\
\sum_{m=1}^{M} \frac{\partial D_{o}\left(x^{*}, y^{*}\right)}{\partial y_{m}} y_{m}^{*}=D_{o}\left(x^{*}, y^{*}\right), \\
\sum_{j=1}^{M} \frac{\partial^{2} D_{o}\left(x^{*}, y^{*}\right)}{\partial y_{j} \partial y_{k}} y_{j}^{*}=0, \quad \forall k \in[1, \ldots, M], \\
\frac{\partial^{2} D_{o}\left(x^{*}, y^{*}\right)}{\partial x_{n} \partial y_{m}} y_{m}^{*}=\frac{\partial D_{o}\left(x^{*}, y^{*}\right)}{\partial x_{n}}, \quad \forall n \in[1, \ldots, N] .
\end{gathered}
$$

${ }^{26}$ Technology exhibits constant returns to scale if $(x, y) \in T^{t}$ implies $(\lambda x, \lambda y) \in T^{t}$ for all $\lambda>0$. 
The period $t$ input distance function $D_{i}^{t}: \mathbb{R}_{+}^{M+N} \rightarrow \mathbb{R}_{+} \cup\{+\infty\}$ is another characterization of the technology of period $t .{ }^{27}$ It is defined as:

$$
D_{i}^{t}(y, x) \equiv \sup \left\{\theta>0: x / \theta \in L^{t}(y)\right\}
$$

It measures the radial distance from inputs $x$ to the input isoquant of period $t$ by the maximum contraction of $x$. Therefore, the following equation is a necessary and sufficient condition for inputs $x$ to be on the input isoquant of $L^{t}(y)$ :

$$
D_{i}^{t}(y, x)=1
$$

By construction, the input distance function is linearly homogeneous in inputs, so that:

$$
\lambda D_{i}^{t}(y, x)=D_{i}^{t}(y, \lambda x), \quad \forall \lambda>0 \text { and } \forall(x, y) \in \mathbb{R}_{+}^{N+M} .
$$

Since we assume constant returns to scale technology, the input distance function is homogeneous of degree minus one in outputs, so that:

$$
\lambda^{-1} D_{i}^{t}(y, x)=D_{i}^{t}(\lambda y, x), \quad \forall \lambda>0 \text { and } \forall(x, y) \in \mathbb{R}_{+}^{N+M}
$$

Thus, under constant returns to scale technology, the input distance function is a homogeneous function with respect to outputs as well as inputs. By applying the Euler's theorem on differentiable homogeneous functions, we can derive the next lemma, which is the counterpart of Lemma 1 for the input distance function.

Lemma 2 (Diewert 1976): Suppose that the technology exhibits constant returns to scale and the input distance function $D_{i}$ is twice differentiable at $\left(x^{*}, y^{*}\right)$. Then, $D_{i}$ satisfies the following equations:

$$
\begin{gathered}
\sum_{n=1}^{N} \frac{\partial D_{i}\left(y^{*}, x^{*}\right)}{\partial x_{n}} x_{n}^{*}=D_{i}\left(y^{*}, x^{*}\right), \\
\sum_{v=1}^{N} \frac{\partial^{2} D_{i}\left(y^{*}, x^{*}\right)}{\partial x_{u} \partial x_{v}} x_{v}^{*}=0, \quad \forall u \in[1, \ldots, N], \\
\sum_{n=1}^{N} \frac{\partial^{2} D_{i}\left(y^{*}, x^{*}\right)}{\partial x_{n} \partial y_{m}} x_{n}^{*}=\frac{\partial D_{i}\left(y^{*}, x^{*}\right)}{\partial y_{m}}, \quad \forall m \in[1, \ldots, M], \\
-\sum_{m=1}^{M} \frac{\partial D_{i}\left(y^{*}, x^{*}\right)}{\partial y_{m}} y_{m}^{*}=D_{i}\left(y^{*}, x^{*}\right),
\end{gathered}
$$

\footnotetext{
${ }^{27}$ See Shephard (1953).
} 


$$
\begin{gathered}
\sum_{j=1}^{M} \frac{\partial^{2} D_{i}\left(y^{*}, x^{*}\right)}{\partial y_{j} \partial y_{k}} y_{j}^{*}=-2 \frac{\partial D_{i}\left(y^{*}, x^{*}\right)}{\partial y_{k}}, \quad \forall k \in[1, \ldots, M] \\
\sum_{m=1}^{M} \frac{\partial^{2} D_{i}\left(y^{*}, x^{*}\right)}{\partial x_{n} \partial y_{m}} y_{m}^{*}=-\frac{\partial D_{i}\left(y^{*}, x^{*}\right)}{\partial x_{n}}, \forall n \in[1, \ldots, N] .
\end{gathered}
$$

Distance functions are convenient tools for characterizing the underlying technology and are the key instruments for constructing theoretical indexes related to producers. Caves et al. (1982) introduce the Malmquist indexes of input, output and productivity by utilizing distance functions. ${ }^{28}$

The period $t$ Malmquist output index compares the radial distances of the output vectors in two periods, relative to the technology of the period $t$, conditional on the input vector of the period $t$ for $t=0,1$, and is defined as:

$$
\operatorname{MOI}^{t} \equiv \frac{D_{o}^{t}\left(x^{t}, y^{1}\right)}{D_{o}^{t}\left(x^{t}, y^{0}\right)}
$$

The period $t$ Malmquist input index compares the radial distances of the input vectors in two periods, relative to the technology of the period $t$, conditional on the output vector of the period $t$ for $t=0,1$, and is defined as:

$$
M I I^{t} \equiv \frac{D_{i}^{t}\left(y^{t}, x^{1}\right)}{D_{i}^{t}\left(y^{t}, x^{0}\right)} .
$$

The output-oriented period $t$ Malmquist productivity index (the period $t$ Malmquist productivity index, hereafter) compares the radial distances of the output and input vectors in two periods, relative to the technology of period $t$ for $t=0,1 .^{29} \mathrm{It}$ is defined as:

$$
M P I^{t} \equiv \frac{D_{o}^{t}\left(x^{1}, y^{1}\right)}{D_{o}^{t}\left(x^{0}, y^{0}\right)}
$$

In the single input-single output case, productivity is the ratio of output over input and the productivity growth between two periods is measured by the firm's output ratio divided by its input ratio. The Hicks-Moorsteen productivity index, which is another

\footnotetext{
${ }^{28}$ It is possible that for some $(x, y) \in T^{t}$, we have $D_{o}^{t}(x, y)=0$ or $D_{i}^{t}(y, x)=0$ or $D_{o}^{t}(x, y)=+\infty$ or $D_{i}^{t}(y, x)=+\infty$. We rule out these extreme cases so as to ensure that the distance functions are welldefined and they can be used as components of theoretical indexes.

${ }^{29}$ While Caves et al. (1982) define the input-oriented Malmquist productivity by $D_{i}^{t}\left(x^{0}, y^{0}\right) / D_{i}^{t}\left(x^{1}, y^{1}\right)$, Färe and Grosskopf (1996) define it by $D_{i}^{t}\left(x^{1}, y^{1}\right) / D_{i}^{t}\left(x^{0}, y^{0}\right)$. Our result on the output-oriented Malmquist index is directly applicable to the input-oriented Malmquist productivity index, no matter which definition is adopted. We focus on the output-oriented Malmquist productivity index to avoid unnecessary repetitions.
} 
popular productivity index formulated by Bjurek (1996), directly generalizes this ratio by utilizing the Malmquist output and input indexes. ${ }^{30}$ The period $t$ Hicks-Moorsteen productivity index is constructed by the period $t$ Malmquist output and input indexes for $t=0,1$. It is defined as:

$$
H M P I^{t} \equiv \frac{M O I^{t}}{M I I^{t}}
$$

All these theoretical indexes are defined for the reference technology $t$. Since periods 0 and 1 are both reasonable candidates as the reference, Caves et al. (1982) and Bjurek (1996) follow Fisher's approach and adopt the geometric mean of period 0 index and period 1 index to avoid the arbitrary choice of the reference technology. ${ }^{31}$ The Malmquist output index $(M O I)$, the Malmquist input index $(M I I)$, the Malmquist productivity index $(M P I)$ and the Hicks-Moorsteen productivity index (HMPI) are, respectively, defined as follows:

$$
\begin{aligned}
M O I & \equiv \sqrt{M O I^{0} \cdot M O I^{1}} . \\
M I I & \equiv \sqrt{M I I^{0} \cdot M I I^{1}} . \\
M P I & \equiv \sqrt{M P I^{0} \cdot M P I^{1}} . \\
H M P I & \equiv \sqrt{H M P I^{0} \cdot H M P I^{1}} .
\end{aligned}
$$

All these theoretical indexes are defined by arbitrary distance functions, which are typically unobserved and unspecified. Thus, one often needs to assume a certain parametric form and estimate the parameters for the underlying distance functions in order to implement these indexes. On the other hand, the indexes in the next sub-section are empirical index number formulae of prices and quantities of inputs and outputs. Thus, they are directly computable from price and quantity observations without estimating distance functions.

\subsection{Empirical Index Number Formulae}

An empirical index number is a formula of prices and quantities of outputs and inputs. Once price and quantity observations are available, they are directly computable. Consider that outputs $y=\left(y_{1}, \ldots, y_{M}\right) \in \mathbb{R}_{++}^{M}$ are sold at prices $p=\left(p_{1}, \ldots, p_{M}\right) \in$ $\mathbb{R}_{++}^{M}$ and inputs $x=\left(x_{1}, \ldots, x_{N}\right) \in \mathbb{R}_{++}^{N}$ are purchased at prices $w=\left(w_{1}, \ldots, w_{N}\right) \in$ $\mathbb{R}_{++}^{N}$ (all strictly positive).

The present paper deals with the quadratic-mean-of-order- $r$ output index, which is a quantity index introduced by Diewert (1976). It is defined for arbitrary non-zero real number $r$ as:

\footnotetext{
${ }^{30}$ The idea of measuring productivity growth by the ratio of the Malmquist output and input indexes is originally proposed by Diewert (1992).

${ }^{31}$ Diewert and Fox (2017) show that the equally-weighted geometric mean such as MOI, MII and HMPI is the only homogeneous mean that satisfies the desirable time reversal property.
} 


$$
Q M O I_{r} \equiv\left(\frac{\sum_{m=1}^{M}\left(\frac{p_{m}^{0} y_{m}^{0}}{\sum_{j=1}^{M} p_{j}^{0} y_{j}^{0}}\right)\left(\frac{y_{m}^{1}}{y_{m}^{0}}\right)^{r / 2}}{\sum_{m=1}^{M}\left(\frac{p_{m}^{1} y_{m}^{1}}{\sum_{j=1}^{M} p_{j}^{1} y_{j}^{1}}\right)\left(\frac{y_{m}^{0}}{y_{m}^{1}}\right)^{r / 2}}\right)^{1 / r}
$$

Since we can freely choose $r$, this is a family of output indexes, which embraces many well-known indexes in the literature of index number theory. In particular, it yields the implicit Walsh output index (IWOI) for $r=1$ and the Fisher output index (FOI) for $r=2 .^{32}$ Two more indexes can be covered as limiting cases. Letting $r \rightarrow 0$ makes it converge to the Törnqvist output index (TOI) and letting $|r| \rightarrow \infty$ makes it converge to the geometric mean of maximum and minimum output relatives (GMOI). These indexes are defined respectively as follows:

$$
\begin{aligned}
& I W O I \equiv Q M O I_{1}=\left(\frac{\sum_{m=1}^{M} p_{m}^{1} y_{m}^{1}}{\sum_{m=1}^{M} p_{m}^{0} y_{m}^{0}}\right) /\left(\frac{\sum_{m=1}^{M}\left(y_{m}^{0} y_{m}^{1}\right)^{1 / 2} p_{m}^{1}}{\sum_{m=1}^{M}\left(y_{m}^{0} y_{m}^{1}\right)^{1 / 2} p_{m}^{0}}\right) \\
& F O I \equiv Q M O I_{2}=\left(\frac{\sum_{m=1}^{M} p_{m}^{0} y_{m}^{1}}{\sum_{m=1}^{M} p_{m}^{0} y_{m}^{0}} \cdot \frac{\sum_{m=1}^{M} p_{m}^{1} y_{m}^{1}}{\sum_{m=1}^{M} p_{m}^{1} y_{m}^{0}}\right)^{1 / 2} \text {. } \\
& T O I \equiv \lim _{r \rightarrow 0} Q M O I_{r}=\prod_{m=1}^{M}\left(\frac{y_{m}^{1}}{y_{m}^{0}}\right)^{\frac{1}{2}\left(p_{m}^{0} y_{m}^{0} / \sum_{j=1}^{M} p_{j}^{0} y_{j}^{0}+p_{m}^{1} y_{m}^{1} / \sum_{j=1}^{M} p_{j}^{1} y_{j}^{1}\right)} \text {. } \\
& G M O I \equiv \lim _{r \rightarrow+\infty} Q M O I_{r} \equiv \lim _{r \rightarrow-\infty} Q M O I_{r} \\
& =\left(\min _{m \in[1, \cdots, M]}\left(\frac{y_{m}^{1}}{y_{m}^{0}}\right) \max _{m \in[1, \cdots, M]}\left(\frac{y_{m}^{1}}{y_{m}^{0}}\right)\right)^{1 / 2} .
\end{aligned}
$$

The term 'implicit output Walsh index' comes from the fact that IWOI equals the revenue ratio between two periods deflated by the Walsh output price index $\sum_{m=1}^{M}\left(y_{m}^{0} y_{m}^{1}\right)^{1 / 2} p_{m}^{1} / \sum_{m=1}^{M}\left(y_{m}^{0} y_{m}^{1}\right)^{1 / 2} p_{m}^{0}$. The Walsh output index $(W O I)$ is the direct index counterpart of IWOI. It is defined as follows:

$$
W O I \equiv \frac{\sum_{m=1}^{M}\left(p_{m}^{0} p_{m}^{1}\right)^{1 / 2} y_{m}^{1}}{\sum_{m=1}^{M}\left(p_{m}^{0} p_{m}^{1}\right)^{1 / 2} y_{m}^{0}}
$$

Similarly, the present paper also deals with the quadratic-mean-of-order- $r$ input index. It is defined for arbitrary non-zero real number $r$ as:

$$
Q M I I_{r} \equiv\left(\frac{\sum_{n=1}^{N}\left(\frac{w_{n}^{0} x_{n}^{0}}{\sum_{u=1}^{N} w_{u}^{0} x_{u}^{0}}\right)\left(\frac{x_{n}^{1}}{x_{n}^{0}}\right)^{r / 2}}{\sum_{n=1}^{N}\left(\frac{w_{n}^{1} x_{n}^{1}}{\sum_{u=1}^{N} w_{u}^{1} x_{u}^{1}}\right)\left(\frac{x_{n}^{0}}{x_{n}^{1}}\right)^{r / 2}}\right)^{1 / r} .
$$

\footnotetext{
${ }^{32}$ See Allen and Diewert (1981) for more on the latter.
} 
Since we can freely choose $r$, this is a family of input indexes, which incorporates many well-known indexes in the literature of index number theory. In particular, the implicit Walsh input index (IWII) and the Fisher input index (FII) can be regarded as special cases of this family. Similarly, the Törnqvist input index (TII) and the geometric mean of maximum and minimum input relatives $(G M I I)$ can also be regarded as its limiting cases. These indexes are defined respectively as follows:

$$
\begin{gathered}
I W I I \equiv Q M I I_{1}=\left(\frac{\sum_{n=1}^{N} w_{n}^{1} x_{n}^{1}}{\sum_{n=1}^{N} w_{n}^{0} x_{n}^{0}}\right) /\left(\frac{\sum_{n=1}^{N}\left(x_{n}^{0} x_{n}^{1}\right)^{1 / 2} w_{n}^{1}}{\sum_{n=1}^{N}\left(x_{n}^{0} x_{n}^{1}\right)^{1 / 2} w_{n}^{0}}\right) . \\
F I I \equiv Q M I I_{2}=\left(\frac{\sum_{n=1}^{N} w_{n}^{0} x_{n}^{1}}{\sum_{n=1}^{N} w_{n}^{0} x_{n}^{0}} \cdot \frac{\sum_{n=1}^{N} w_{n}^{1} x_{n}^{1}}{\sum_{n=1}^{N} w_{n}^{1} x_{n}^{0}}\right)^{1 / 2} \cdot \\
T I I \equiv \lim _{r \rightarrow 0} Q M I I_{r}=\prod_{n=1}^{N}\left(\frac{x_{n}^{1}}{x_{n}^{0}}\right)^{\frac{1}{2}\left(w_{n}^{0} x_{n}^{0} / \sum_{u=1}^{N} w_{u}^{0} x_{u}^{0}+w_{n}^{1} x_{n}^{1} / \sum_{u=1}^{N} w_{u}^{1} x_{u}^{1}\right)} . \\
G M I I \equiv \lim _{r \rightarrow+\infty} Q M I I_{r} \equiv \lim _{r \rightarrow-\infty} Q M I I_{r} \\
=\left(\min _{n \in[1, \cdots, N]}\left(\frac{x_{n}^{1}}{x_{n}^{0}}\right) \max _{n \in[1, \cdots, N]}\left(\frac{x_{n}^{1}}{x_{n}^{0}}\right)\right)^{1 / 2} .
\end{gathered}
$$

The term 'implicit input Walsh index' comes from the fact that IWII equals the cost ratio between two periods deflated by the Walsh input price index $\sum_{n=1}^{N}\left(x_{n}^{0} x_{n}^{1}\right)^{1 / 2} w_{n}^{1} / \sum_{n=1}^{N}\left(x_{n}^{0} x_{n}^{1}\right)^{1 / 2} w_{n}^{0}$. The Walsh input index $(W I I)$ is the direct index counterpart of IWII. It is defined as follows:

$$
W I I \equiv \frac{\sum_{n=1}^{N}\left(w_{n}^{0} w_{n}^{1}\right)^{1 / 2} x_{n}^{1}}{\sum_{n=1}^{N}\left(w_{n}^{0} w_{n}^{1}\right)^{1 / 2} x_{n}^{0}} .
$$

A productivity index is often defined as the ratio of output index to input index. Thus, we can introduce the quadratic-mean-of-order- $r$ productivity index, which is the ratio of the quadratic-mean-of-order- $r$ output index to the quadratic-mean-of-order- $r$ input index, for arbitrary non-zero real number $r$. It is defined as:

$$
Q M P I_{r} \equiv \frac{\left(\frac{\sum_{m=1}^{M}\left(\frac{p_{m}^{0} y_{m}^{0}}{\sum_{j=1}^{M} p_{j}^{0} y_{j}^{0}}\right)\left(\frac{y_{m}^{1}}{y_{m}^{0}}\right)^{r / 2}}{\sum_{m=1}^{M}\left(\frac{p_{m}^{1} y_{m}^{1}}{\sum_{j=1}^{M} p_{j}^{1} y_{j}^{1}}\right)\left(\frac{y_{m}^{0}}{y_{m}^{1}}\right)^{r / 2}}\right)^{1 / r}}{\left(\frac{\sum_{n=1}^{N}\left(\frac{w_{n}^{0} x_{n}^{0}}{\sum_{u=1}^{N} w_{u}^{0} x_{u}^{0}}\right)\left(\frac{x_{n}^{1}}{x_{n}^{0}}\right)^{r / 2}}{\sum_{n=1}^{N}\left(\frac{w_{n}^{1} x_{n}^{1}}{\sum_{u=1}^{N} w_{u}^{1} x_{u}^{1}}\right)\left(\frac{x_{n}^{0}}{x_{n}^{1}}\right)^{r / 2}}\right)^{1 / r}}
$$


Again, since we can freely choose $r$, this is a family of productivity indexes, which incorporates many well-known indexes in the literature of index number theory. In particular, the implicit Walsh productivity index (IWPI) and the Fisher productivity index $(F P I)$ can be regarded as special cases of this family. Similarly, the Törnqvist productivity index (TPI) and the ratio of the geometric mean of maximum and minimum output relatives to the geometric mean of maximum and minimum input relatives (GMPI) can also be regarded as its limiting cases. These indexes are defined respectively as follows:

$$
\begin{gathered}
I W P I \equiv Q M P I_{1}=\frac{\left(\left(\frac{\sum_{m=1}^{M} p_{m}^{1} y_{m}^{1}}{\sum_{m=1}^{M} p_{m}^{0} y_{m}^{0}}\right) /\left(\frac{\sum_{m=1}^{M}\left(y_{m}^{0} y_{m}^{1}\right)^{1 / 2} p_{m}^{1}}{\sum_{m=1}^{M}\left(y_{m}^{0} y_{m}^{1}\right)^{1 / 2} p_{m}^{0}}\right)\right)}{\left(\left(\frac{\sum_{n=1}^{N} w_{n}^{1} x_{n}^{1}}{\sum_{n=1}^{N} w_{n}^{0} x_{n}^{0}}\right) /\left(\frac{\sum_{n=1}^{N}\left(x_{n}^{0} x_{n}^{1}\right)^{1 / 2} w_{n}^{1}}{\sum_{n=1}^{N}\left(x_{n}^{0} x_{n}^{1}\right)^{1 / 2} w_{n}^{0}}\right)\right)} . \\
F P I \equiv Q M P I_{2}=\frac{\left(\frac{\sum_{m=1}^{M} p_{m}^{0} y_{m}^{1}}{\sum_{m=1}^{M} p_{m}^{0} y_{m}^{0}} \cdot \frac{\sum_{m=1}^{M} p_{m}^{1} y_{m}^{1}}{\sum_{m=1}^{M} p_{m}^{1} y_{m}^{0}}\right)^{1 / 2}}{\left(\frac{\sum_{n=1}^{N} w_{n}^{0} x_{n}^{1}}{\sum_{n=1}^{N} w_{n}^{0} x_{n}^{0}} \cdot \frac{\sum_{n=1}^{N} w_{n}^{1} x_{n}^{1}}{\sum_{n=1}^{N} w_{n}^{1} x_{n}^{0}}\right)^{1 / 2}} . \\
=\frac{T P I \equiv \lim _{r \rightarrow 0} Q M P I_{r}}{\prod_{m=1}^{M}\left(\frac{y_{m}^{1}}{y_{m}^{0}}\right)^{\frac{1}{2}\left(p_{m}^{0} y_{m}^{0} / \sum_{j=1}^{M} p_{j}^{0} y_{j}^{0}+p_{m}^{1} y_{m}^{1} / \sum_{j=1}^{M} p_{j}^{1} y_{j}^{1}\right)}} \\
G M P I \equiv \lim _{r \rightarrow+\infty}\left(\frac{x_{n}^{1}}{x_{n}^{0}}\right)^{\frac{1}{2}\left(w_{n}^{0} x_{n}^{0} / \sum_{u=1}^{N} w_{u}^{0} \cdot x_{u}^{0}+w_{n}^{1} x_{n}^{1} / \sum_{u=1}^{N} w_{u}^{1} \cdot x_{u}^{1}\right)} \\
Q M P I_{r} \equiv \lim _{r \rightarrow-\infty} Q M P I_{r} \\
=\frac{\left.\min _{m \in[1, \cdots, M]}\left(\frac{y_{m}^{1}}{y_{m}^{0}}\right)_{m \in[1, \cdots, M]}\left(\frac{y_{m}^{1}}{y_{m}^{0}}\right)\right)^{1 / 2}}{\left(\min _{n \in[1, \cdots, N]}\left(\frac{x_{n}^{1}}{x_{n}^{0}}\right)_{n \in[1, \cdots, N]}\left(\frac{x_{n}^{1}}{x_{n}^{0}}\right)\right)^{1 / 2}} .
\end{gathered}
$$

As the case of output and input indexes, we can define the Walsh productivity index $(W P I)$, which is based on the Walsh output and input indexes (WOI) and (WII), as follows:

$$
W P I \equiv \frac{\left(\frac{\sum_{m=1}^{M}\left(p_{m}^{0} p_{m}^{1}\right)^{1 / 2} y_{m}^{1}}{\sum_{m=1}^{M}\left(p_{m}^{0} p_{m}^{1}\right)^{1 / 2} y_{m}^{0}}\right)}{\left(\frac{\sum_{n=1}^{N}\left(w_{n}^{0} w_{n}^{1}\right)^{1 / 2} x_{n}^{1}}{\sum_{n=1}^{N}\left(w_{n}^{0} w_{n}^{1}\right)^{1 / 2} x_{n}^{0}}\right)}
$$

\section{Axiomatic Properties and Implicit Walsh Index}

Axiomatic properties for a variety of price and quantity indexes have been examined in the literature of index number theory. The International Labour Office et al. (2004), also referred to as 'CPI Manual', is the standard reference source for the consumer price 
index in terms of theory and practice. They deal with the following 20 tests (axioms), which have been proposed in the past in the literature of the index number theory. ${ }^{33}$ While all the empirical indexes of output and input introduced in the previous section are quantity indexes, International Labour Office et al. (2004) focus on the axioms for price indexes. Thus, we provide the corresponding axioms for quantity indexes below.

We denote an arbitrary output index by $O I\left(p^{0}, p^{1}, y^{0}, y^{1}\right)$, so as to emphasize that the empirical output index is a function of price and quantity vector of two periods being compared. ${ }^{34}$ Every test for output index in this list has a direct counterpart for input, which we omit to avoid unnecessary repetition.

T1. Positivity: $O I\left(p^{0}, p^{1}, y^{0}, y^{1}\right)>0$.

T2. Continuity: $O I\left(p^{0}, p^{1}, y^{0}, y^{1}\right)$ is a continuous function of its arguments.

T3. Identity or constant quantities test: $O I\left(p^{0}, p^{1}, y, y\right)=1$.

T4. Fixed basket or constant prices test: $O I\left(p, p, y^{0}, y^{1}\right)=\sum_{m=1}^{M} p_{m} y_{m}^{1} / \sum_{m=1}^{M} p_{m} y_{m}^{0}$.

T5. Proportionality in current quantities:

$$
O I\left(p^{0}, p^{1}, y^{0}, \lambda y^{1}\right)=\lambda O I\left(p^{0}, p^{1}, y^{0}, y^{1}\right) \text { for all } \lambda>0 .
$$

T6. Inverse proportionality in base period quantities:

$$
O I\left(p^{0}, p^{1}, \lambda y^{0}, y^{1}\right)=\lambda^{-1} O I\left(p^{0}, p^{1}, y^{0}, y^{1}\right) \text { for all } \lambda>0 \text {. }
$$

T7. Invariance to proportional changes in current prices:

$$
O I\left(p^{0}, \lambda p^{1}, y^{0}, y^{1}\right)=O I\left(p^{0}, p^{1}, y^{0}, y^{1}\right) \text { for all } \lambda>0 \text {. }
$$

T8. Invariance to proportional changes in base prices:

$$
O I\left(\lambda p^{0}, p^{1}, y^{0}, y^{1}\right)=O I\left(p^{0}, p^{1}, y^{0}, y^{1}\right) \text { for all } \lambda>0 \text {. }
$$

T9. Commodity reversal test: $O I\left(p^{0^{*}}, p^{1^{*}}, y^{0^{*}}, y^{1^{*}}\right)=O I\left(p^{0}, p^{1}, y^{0}, y^{1}\right)$ where $p^{t^{*}}$ denotes a permutation of the components of the vector $p^{t}$ and $y^{t^{*}}$ denotes the same permutation of the components of $y^{t}$ for $t=0,1$.

T10. Invariance to changes in the units of measurement:

$$
\begin{aligned}
& O I\left(\alpha_{1} p_{1}^{0}, \ldots, \alpha_{M} p_{M}^{0}, \alpha_{1} p_{1}^{1}, \ldots, \alpha_{M} p_{M}^{1}, \alpha_{1}^{-1} y_{1}^{0}, \ldots, \alpha_{M}^{-1} y_{M}^{0}, \alpha_{1}^{-1} y_{1}^{1}, \ldots, \alpha_{M}^{-1} y_{M}^{1}\right) \\
& \quad=O I\left(p_{1}^{0}, \ldots, p_{M}^{0}, p_{1}^{1}, \ldots, p_{M}^{1}, y_{1}^{0}, \ldots, y_{M}^{0}, y_{1}^{1}, \ldots, y_{M}^{1}\right) \text { for all } \alpha=\left(\alpha_{1}, \ldots, \alpha_{M}\right)>0 .
\end{aligned}
$$

T11. Time reversal test: $O I\left(p^{0}, p^{1}, y^{0}, y^{1}\right)=1 / O I\left(p^{1}, p^{0}, y^{1}, y^{0}\right)$

T12. Price reversal test: $O I\left(p^{0}, p^{1}, y^{0}, y^{1}\right)=O I\left(p^{1}, p^{0}, y^{0}, y^{1}\right)$

T13. Quantity reversal test:

$$
\left(\frac{\sum_{m=1}^{M} p_{m}^{1} y_{m}^{1}}{\sum_{m=1}^{M} p_{m}^{0} y_{m}^{0}}\right) / O I\left(p^{0}, p^{1}, y^{0}, y^{1}\right)=\left(\frac{\sum_{m=1}^{M} p_{m}^{1} y_{m}^{0}}{\sum_{m=1}^{M} p_{m}^{0} y_{m}^{1}}\right) / O I\left(p^{0}, p^{1}, y^{1}, y^{0}\right)
$$

T14. Mean value test for quantities:

\footnotetext{
${ }^{33}$ Also see Diewert (1992), which the International Labour Office et al. (2004) follow.

${ }^{34}$ We sometimes adopt this type of notation for a specific index. For example, the Fisher, the implicit Walsh, the quadratic-mean-of-order- $r$ and the Malmquist output index applied to observations in periods 0 and 1 can be denoted by $\operatorname{FOI}\left(p^{1}, p^{0}, y^{0}, y^{1}\right), \operatorname{IWOI}\left(p^{0}, p^{1}, y^{0}, y^{1}\right), \operatorname{QMOI}_{r}\left(p^{0}, p^{1}, y^{0}, y^{1}\right)$ and $\operatorname{MOI}\left(x^{0}, x^{1}, y^{0}, y^{1}\right)$, as an arbitrary output index denoted by $O I\left(p^{0}, p^{1}, y^{0}, y^{1}\right)$.
} 


$$
\min \left(\frac{y_{1}^{1}}{y_{1}^{0}}, \ldots, \frac{y_{M}^{1}}{y_{M}^{0}}\right) \leq O I\left(p^{0}, p^{1}, y^{0}, y^{1}\right) \leq \max \left(\frac{y_{1}^{1}}{y_{1}^{0}}, \ldots, \frac{y_{M}^{1}}{y_{M}^{0}}\right) .
$$

T15. Mean value test for prices:

$$
\min \left(\frac{p_{1}^{1}}{p_{1}^{0}}, \ldots, \frac{p_{M}^{1}}{p_{M}^{0}}\right) \leq\left(\frac{\sum_{m=1}^{M} p_{m}^{1} y_{m}^{1}}{\sum_{m=1}^{M} p_{m}^{0} y_{m}^{0}}\right) / O I\left(p^{0}, p^{1}, y^{0}, y^{1}\right) \leq \max \left(\frac{p_{1}^{1}}{p_{1}^{0}}, \ldots, \frac{p_{M}^{1}}{p_{M}^{0}}\right) .
$$

T16. Paasche and Laspeyres bounding test: output index $O I\left(p^{0}, p^{1}, y^{0}, y^{1}\right)$ lies between the Laspeyres and Paasche indexes, so that either of the following inequalities holds:

$\frac{\sum_{m=1}^{M} p_{m}^{0} y_{m}^{1}}{\sum_{m=1}^{M} p_{m}^{0} y_{m}^{0}} \leq O I\left(p^{0}, p^{1}, y^{0}, y^{1}\right) \leq \frac{\sum_{m=1}^{M} p_{m}^{1} y_{m}^{1}}{\sum_{m=1}^{M} p_{m}^{1} y_{m}^{0}} \quad$ or $\quad \frac{\sum_{m=1}^{M} p_{m}^{1} y_{m}^{1}}{\sum_{m=1}^{M} p_{m}^{1} y_{m}^{0}} \leq O I\left(p^{0}, p^{1}, y^{0}, y^{1}\right) \leq$ $\frac{\sum_{m=1}^{M} p_{m}^{0} y_{m}^{1}}{\sum_{m=1}^{M} p_{m}^{0} y_{m}^{0}}$

T17. Monotonicity in current quantities: $O I\left(p^{0}, p^{1}, y^{0}, y^{1}\right)<O I\left(p^{0}, p^{1}, y^{0}, y^{2}\right)$ if $y^{1}<y^{2}$.

T18. Monotonicity in base quantities: $O I\left(p^{0}, p^{1}, y^{0}, y^{1}\right)>O I\left(p^{0}, p^{1}, y^{2}, y^{1}\right)$ if $y^{0}<$ $y^{2}$.

T19. Monotonicity in current prices:

$\left(\frac{\sum_{m=1}^{M} p_{m}^{1} y_{m}^{1}}{\sum_{m=1}^{M} p_{m}^{0} y_{m}^{0}}\right) / O I\left(p^{0}, p^{1}, y^{0}, y^{1}\right)<\left(\frac{\sum_{m=1}^{M} p_{m}^{2} y_{m}^{1}}{\sum_{m=1}^{M} p_{m}^{0} y_{m}^{0}}\right) / O I\left(p^{0}, p^{2}, y^{0}, y^{1}\right)$ if $p^{1}<p^{2}$

T20. Monotonicity in base prices:

$\left(\frac{\sum_{m=1}^{M} p_{m}^{1} y_{m}^{1}}{\sum_{m=1}^{M} p_{m}^{0} y_{m}^{0}}\right) / O I\left(p^{0}, p^{1}, y^{0}, y^{1}\right)>\left(\frac{\sum_{m=1}^{M} p_{m}^{1} y_{m}^{1}}{\sum_{m=1}^{M} p_{m}^{2} y_{m}^{0}}\right) / O I\left(p^{2}, p^{1}, y^{0}, y^{1}\right)$ if $p^{0}<p^{2}$

International Labour Office et al. (2004) contain extensive discussions on the axiomatic properties of several commonly used indexes, such as the Laspeyres, Paasche, Fisher, Törnqvist and Walsh indexes. However, it does not deal with the implicit Walsh index. To the best of our knowledge, no studies have comprehensively examined the implicit Walsh index in terms of the axioms or tests that it satisfies. ${ }^{35}$ Thus, we try to fill this apparent gap in the literature by examining its axiomatic properties below.

Proposition 1: The implicit Walsh output index IWOI satisfies the following 16 tests: T1, T2, T3, T4, T5, T6, T7, T8, T9, T10, T11, T13, T14, T15, T19 and T20. However, it does not satisfy the remaining 4 tests: T12, T16, T17 and T18.

Table 1 compares five widely used indexes as well as the implicit Walsh index in terms of 20 tests. It also reports the number of tests each index satisfies. It summarizes the analysis of International Labour Office et al. (2004) on these five indexes as well as our analysis on the implicit Walsh index.

\footnotetext{
${ }^{35}$ Balk (2008) empirically compares different implicit indexes including the implicit Walsh index.
} 
Table 1: Axiomatic properties of six empirical index number formulae

\begin{tabular}{|c|c|c|c|c|c|c|c|}
\hline & Test & Fisher & Törnqvist & Walsh & implicit Walsh & Laspeyres & Paasche \\
\hline $\mathrm{T} 1$ & Positivity & Yes & Yes & Yes & Yes & Yes & Yes \\
\hline $\mathrm{T} 2$ & Continuity & Yes & Yes & Yes & Yes & Yes & Yes \\
\hline $\mathrm{T} 3$ & Identity or constant quantities test & Yes & Yes & Yes & Yes & Yes & Yes \\
\hline $\mathrm{T} 4$ & Fixed basket or constant prices test & Yes & No & Yes & Yes & Yes & Yes \\
\hline T5 & Proportionality in current quantities & Yes & Yes & Yes & Yes & Yes & Yes \\
\hline T6 & Inverse proportionality in base period quantities & Yes & Yes & Yes & Yes & Yes & Yes \\
\hline $\mathrm{T} 7$ & Invariance to proportional changes in current prices & Yes & Yes & Yes & Yes & Yes & Yes \\
\hline $\mathrm{T} 8$ & Invariance to proportional changes in base prices & Yes & Yes & Yes & Yes & Yes & Yes \\
\hline T9 & Commodity reversal test & Yes & Yes & Yes & Yes & Yes & Yes \\
\hline $\mathrm{T} 10$ & Invariance to changes in the units of measurement & Yes & Yes & Yes & Yes & Yes & Yes \\
\hline T11 & Time reversal test & Yes & Yes & Yes & Yes & No & No \\
\hline $\mathrm{T} 12$ & Price reversal test & Yes & No & Yes & No & No & No \\
\hline $\mathrm{T} 13$ & Quantity reversal test & Yes & No & No & Yes & No & No \\
\hline $\mathrm{T} 14$ & Mean value test for quantities & Yes & Yes & Yes & Yes & Yes & Yes \\
\hline T15 & Mean value test for prices & Yes & No & Yes & Yes & Yes & Yes \\
\hline T16 & Paasche and Laspeyres bounding test & Yes & No & No & No & Yes & Yes \\
\hline $\mathrm{T} 17$ & Monotonicity in current quantities & Yes & No & Yes & No & Yes & Yes \\
\hline $\mathrm{T} 18$ & Monotonicity in base quantities & Yes & No & Yes & No & Yes & Yes \\
\hline T19 & Monotonicity in current prices & Yes & No & No & Yes & Yes & Yes \\
\hline $\mathrm{T} 20$ & Monotonicity in base prices & Yes & No & No & Yes & Yes & Yes \\
\hline Total & & 20 & 11 & 16 & 16 & 17 & 17 \\
\hline
\end{tabular}

Note: The information for the Fisher, Törnqvist, Walsh, Laspeyres and Paasche indexes can be found in Diewert (1992) and International Labour Office et al. (2004). The information for the implicit Walsh index is confirmed by Proposition 1. 
As Diewert (1992) initially shows, the Fisher index can be regarded as the best among all popular indexes since it satisfies all 20 tests. ${ }^{39}$ Although two most commonly used indexes of the Laspeyres and Paasche satisfy 17 out of the 20 tests, they fail to satisfy the essential time reversal test. While the Törnqvist, Walsh and implicit Walsh indexes satisfy a smaller number of tests than the Fisher index, they satisfy the time reversal test. While the Walsh and implicit Walsh indexes satisfy 16 tests, the Törnqvist index satisfies 11 tests. Moreover, these two Walsh indexes satisfy all the tests which the Törnqvist index satisfies. Therefore, the Walsh and implicit Walsh indexes dominate the Törnqvist index in terms of the 20 tests. $^{40}$

An empirical index is sometimes asked to satisfy the so-called circularity test, which is formulated as:

T21. Circularity test: $O I\left(p^{0}, p^{2}, y^{0}, y^{2}\right)=O I\left(p^{0}, p^{1}, y^{0}, y^{1}\right) \times O I\left(p^{1}, p^{2}, y^{1}, y^{2}\right)$.

To the best of our understanding, the mainstream view on this test is that it is too restrictive. Indeed, as Funke et al. (1979) originally shows, the quantity index that satisfies this test T21 must aggregate quantity relatives using fixed weight or fixed basket. ${ }^{41}$ As a result such index may (and often do) lead to significant substitution bias. $^{42}$ This is apparently a reason why International Labour Office et al. (2004) do not include this test in the above 20 tests and do not ask the indexes to satisfy it. ${ }^{43}$ For the same reason, we also focus on only these 20 tests.

\section{Flexible Functional Form}

As we mentioned before, theoretical indexes are defined by using arbitrary distance functions and thus, it is necessary to specify these functions so as to implement these indexes. Many empirical applications assume a certain functional form and then econometrically estimate its parameters.

It is desirable to adopt the functional form that is sufficiently flexible. If we adopt a restricted functional form (e.g., linear and Cobb-Douglas) for distance functions in our case, the pattern of substitutions among inputs and outputs may be severely limited $a$

\footnotetext{
${ }^{39}$ It is worth noting that within the axiomatic studies on empirical index, there exist different points of view. If one regards an empirical index as a function of prices and quantities, the Fisher index can be regarded as 'the best'. If one regards an empirical index as a function of prices and expenditures, or quantities and expenditures, the Törnqvist index can be regarded as 'the best'. If one focuses on the empirical index that appropriately average the basket quantities or prices when aggregating price or quantity relatives, the Walsh index can be regarded as 'the best'. See International Labour Office et al. (2004) for related discussion.

${ }^{40}$ International Labour Office et al. (2004) concluded by saying "The remaining two indices, the Walsh and Törnqvist price index, both satisfy the time reversal test but the Walsh index emerges as being "better" since it passes 16 of the 20 tests whereas the Törnqvist only satisfies 11 tests." The same argument can apply to the implicit Walsh index as well.

${ }^{41}$ Strictly speaking, Funke et al. (1979) deal with only price indexes. However, their result is directly applicable to quantity indexes as well. See Diewert and Fox (2014) for a discussion on the problems with the fixed-weight index.

${ }^{42}$ Most notably, Boskin et al. (1996), also referred to as 'Boskin report', provide clear evidence of substitution bias in the fixed-weight price index.

${ }^{43}$ See page 282 in International Labour Office et al. (2004).
} 
priori. As a result, it may prevent us from capturing the underlying technology accurately, leading to a biased measure of output and input change and productivity growth. ${ }^{44} \mathrm{~A}$ functional form that has a second order approximation property is conventionally referred to as a 'flexible functional form'. ${ }^{45}$ It nicely allows for many types of substitutions among inputs and outputs.

Suppose a functional form $f$ is a flexible functional form for the output distance function. It means that $f$ can approximate an arbitrary output distance function $D_{o}$ to the second order at an arbitrary point at which $f$ is twice continuously differentiable. More specifically, the value, and the first and second derivatives of $f$ can coincide with those of $D_{o}$ at an arbitrary point, by choosing parameters of $f$ appropriately. In addition, $f$ also needs to satisfy (8) and (9), the homogeneity conditions which the output distance function must satisfy by construction. Translog, generalized Leontief and normalized quadratic functional forms are known to be flexible functional forms for the output and input distance functions. ${ }^{46}$

The present paper introduces the following family of functional form $g_{r}$ based on the quadratic-mean-of-order- $r$ for the output distance function. ${ }^{47}$ It is defined for an arbitrary non-zero real number $r$ :

$$
\begin{aligned}
& g_{r}(x, y) \\
& \equiv\left[\left(\sum_{j=1}^{M} \sum_{k=1}^{M} a_{j, k} y_{j}^{r / 2} y_{k}^{r / 2}\right)\left(\sum_{u=1}^{N} \sum_{v=1}^{N} c_{u, v} x_{u}^{r / 2} x_{v}^{r / 2}\right)^{-1}\right. \\
& \left.+\left(\sum_{m=1}^{M} \alpha_{m} y_{m}^{r / 2}\right)\left(\sum_{n=1}^{N} \beta_{n} x_{n}^{-r / 2}\right)\left(\sum_{m=1}^{M} \sum_{n=1}^{N} b_{m, n} y_{m}^{r / 2} x_{n}^{-r / 2}\right)\right]^{1 / r}
\end{aligned}
$$

where

$$
\begin{array}{ll}
a_{j, k}=a_{k, j}, & \forall j, k \in[1, \ldots, M], \\
c_{u, v}=c_{v, u}, & \forall u, v \in[1, \ldots, N] .
\end{array}
$$

It is worth noting that $g_{r}$ is linearly homogeneous in outputs $y$ and homogeneous of degree minus one in inputs $x$ (i.e., assuming a constant returns to scale technology). The functional form $g_{r}$ generalizes the one proposed by Diewert (1992) and is reduced to that for $r=2 .{ }^{48}$ The functional form $g_{r}$ coincides with the generalized Leontief

\footnotetext{
${ }^{44}$ This paper adopts the index number approach to implement theoretical indexes. However, even when we switch to the econometric approach, it is desirable to impose less restrictions on functional forms for output distance functions.

45 The concept of 'flexible functional form' is first introduced by Diewert $(1973,1974)$.

${ }^{46}$ Diewert (1971) shows the generalized Leontief functional form as a flexible functional form. The translog functional form is originally introduced by Christensen et al. (1973). Diewert and Wales (1987) introduce the normalized quadratic functional form and show that it is a flexible functional form.

${ }^{47}$ Quadratic-mean-of-order- $r$ function goes back to McCarthy (1967) and Kadiyala (1972). They adopt it for production function with a single output.

${ }^{48}$ Balk (1998) calls it the Diewert output distance function or simply the Diewert form.
} 
functional form for $r=1 .{ }^{49}$ Also, if $a_{j, k}=0$ for all $j \neq k, c_{u, v}=0$ for all $u \neq v$, $\alpha_{m}=0$ for all $m, \beta_{n}=0$ for all $n$, and $b_{m, n}=0$ for all $m$ and $n, g_{r}$ is reduced to the constant-elasticity-of-substitution (CES) functional form. ${ }^{50}$ However, the limits (as $r$ goes to zero or infinity) of $g_{r}$ are unknown unlike the case of the quadratic-mean-oforder- $r$ indexes such as $Q M O I_{r}, Q M I I_{r}$ and $Q M P I_{r} .{ }^{51}$ The following proposition shows that this functional form $g_{r}$ is a flexible functional form, irrespective of the value of $r$.

Proposition 2: Let $D_{o}^{*}$ be an arbitrary output distance function and let $\left(x^{*}, y^{*}\right) \in \mathbb{R}_{++}^{N+M}$ be an arbitrary point on the production frontier. Suppose that $D_{o}^{*}$ is twice continuously differentiable at $\left(x^{*}, y^{*}\right)$. Then for any $\alpha_{m}$ for $m=1, \ldots M$ and $\beta_{n}$ for $n=1, \ldots N$ satisfying

$$
\sum_{m=1}^{M} \alpha_{m} y_{m}^{* r / 2} \neq 0 \text { and } \sum_{n=1}^{N} \beta_{n} x_{n}^{*-r / 2} \neq 0,
$$

there exist $a_{j, k}$ for $j$ and $k=1, \ldots, M, c_{u, v}$ for $u$ and $v=1, \ldots, N$ and $b_{m, n}$ for $m=$ $1, \ldots, M$ and $n=1, \ldots N$ such that

$$
\begin{gathered}
\sum_{j=1}^{M} \sum_{k=1}^{M} a_{j, k} y_{j}^{* r / 2} y_{k}^{* r / 2}=r / 2, \\
\sum_{u=1}^{N} \sum_{v=1}^{N} c_{u, v} x_{u}^{*} x_{v}^{*}=r / 2, \\
\sum_{m=1}^{M} b_{m, n} y_{m}^{*} r / 2=0, \forall n \in[1, \ldots, N], \\
\sum_{n=1}^{N} b_{m, n} x_{n}^{*-r / 2}=0, \forall m \in[1, \ldots, M],
\end{gathered}
$$

and $g_{r}$ defined by (52)-(54) can provide a second order approximation to $D_{o}^{*}$ at $\left(x^{*}, y^{*}\right) .^{52}$

Generally, it is possible that the restrictions of (55)-(59) limit the selection of the parameters of $g_{r}$ and thus, destroy its flexibility. However, the above proposition shows that even under these restrictions, $g_{r}$ can approximate any arbitrary output distance function $D_{o}^{*}$ to the second order at an arbitrary production plan $\left(x^{*}, y^{*}\right)$ by choosing its parameters in Equation (52) appropriately. Thus, $g_{r}$ is shown to be a flexible functional form with these restrictions. ${ }^{53}$ Moreover, since $g_{r}$ is the family of functional forms, this proposition also implies that every functional form of this family is a flexible functional form. ${ }^{54}$

Similarly, the present paper also introduces the following family of functional form $h_{r}$ based on the quadratic-mean-of-order- $r$ for input distance function. It is defined for arbitrary non-zero real number $r$ :

\footnotetext{
${ }^{49}$ A variety of functional forms for output distance function based on the generalized Leontief functional form are possible with multiple outputs and multiple inputs. The functional form $g_{1}$ is an example of one of these.

${ }^{50}$ See Hasenkamp (1976) for the CES functional form with multiple outputs and multiple inputs.

${ }^{51}$ Diewert (1980) and Hill (2006) derive the limit of the aggregator function based on the quadratic mean of order $r$, as $r$ converges to 0 or infinity. However, since they only deal with a simplified version of $g_{r}$, we cannot deduce the limit of $g_{r}$ from their results.

${ }^{52}$ It is worth noting that this proposition can apply to the distance functions which are not differentiable in some domains, such as the case of a piece-wise linear functional form.

${ }^{53}$ It is worth noting that $g_{r}$, defined by (52)-(54), is already a flexible functional form without imposing any restrictions (55)-(58).

${ }^{54}$ In the special case when $r=2$, Proposition 2 coincides with Theorem 7 of Diewert (1992), where $g_{2}$ was shown to be a flexible functional form.
} 


$$
\begin{aligned}
& h_{r}(y, x) \\
& \equiv\left[\left(\sum_{j=1}^{M} \sum_{k=1}^{M} a_{j, k} y_{j}^{r / 2} y_{k}^{r / 2}\right)^{-1}\left(\sum_{u=1}^{N} \sum_{v=1}^{N} c_{u, v} x_{u}^{r / 2} x_{v}^{r / 2}\right)\right. \\
& \left.+\left(\sum_{m=1}^{M} \alpha_{m} y_{m}^{-r / 2}\right)\left(\sum_{n=1}^{N} \beta_{n} x_{n}^{r / 2}\right)\left(\sum_{m=1}^{M} \sum_{n=1}^{N} b_{m, n} y_{m}^{-r / 2} x_{n}^{r / 2}\right)\right]^{1 / r}
\end{aligned}
$$

where

$$
\begin{array}{ll}
a_{j, k}=a_{k, j}, & \forall j, k \in[1, \ldots, M], \\
c_{u, v}=c_{v, u}, & \forall u, v \in[1, \ldots, N] .
\end{array}
$$

It is worth noting that $h_{r}$ is linearly homogeneous in inputs $x$ and homogeneous of degree minus one in outputs $y$. The functional form $h_{r}$ generalizes the one proposed by Diewert (1992) and is reduced to it for $r=2 .{ }^{55}$ It includes other popular functional forms such as the generalized Leontief as well as the constant-elasticity-of-substitution (CES) functional forms as special cases. ${ }^{56}$ The following proposition shows that this functional form is a flexible functional form, irrespective of the value of $r$.

Proposition 3: Let $D_{i}^{*}$ be an arbitrary input distance function and let $\left(x^{*}, y^{*}\right) \in \mathbb{R}_{++}^{N+M}$ be an arbitrary point on the production frontier. Suppose that $D_{i}^{*}$ is twice continuously differentiable at $\left(x^{*}, y^{*}\right)$. Then for any $\alpha_{m}$ for $m=1, \ldots, M$ and $\beta_{n}$ for $n=1, \ldots, N$ satisfying

$$
\sum_{m=1}^{M} \alpha_{m} y_{m}^{*-r / 2} \neq 0 \text { and } \sum_{n=1}^{N} \beta_{n} x_{n}^{* r / 2} \neq 0,
$$

there exist $a_{j, k}$ for $j$ and $k=1, \ldots, M, c_{u, v}$ for $u$ and $v=1, \ldots, N$ and $b_{m, n}$ for $m=$ $1, \ldots, M$ and $n=1, \ldots, N$ such that

$$
\begin{gathered}
\sum_{j=1}^{M} \sum_{k=1}^{M} a_{j, k} y_{j}^{* r / 2} y_{k}^{* r / 2}=r / 2, \\
\sum_{u=1}^{N} \sum_{v=1}^{N} c_{u, v} x_{u}^{* r / 2} x_{v}^{* r / 2}=r / 2, \\
\sum_{m=1}^{M} b_{m, n} y_{m}^{*}-r / 2=0, \forall n \in[1, \ldots, N], \\
\sum_{n=1}^{N} b_{m, n} x_{n}^{* r / 2}=0, \forall m \in[1, \ldots, M] .
\end{gathered}
$$

and $h_{r}$ defined by (60)-(62) can provide a second order approximation to $D_{i}^{*}$ at $\left(x^{*}, y^{*}\right){ }^{57}$

\footnotetext{
${ }_{55}^{55}$ Balk (1998) calls it the Diewert input distance function or simply Diewert form.

${ }^{56}$ A variety of functional forms for input distance function based on the generalized Leontief functional form are possible with multiple outputs and multiple inputs. The functional form $h_{1}$ is an example of one of these.

${ }^{57}$ It is worth noting that this proposition can apply to the distance functions which are not differentiable in some domains, such as the case of a piece-wise linear functional form.
} 
Like Proposition 2, the above proposition also shows that even under these restrictions (63)-(67), $h_{r}$ can approximate any arbitrary input distance function $D_{i}^{*}$ to the second order at an arbitrary production plan $\left(x^{*}, y^{*}\right)$. Thus, $h_{r}$ is shown to be a flexible functional form with these restrictions. ${ }^{58}$ Moreover, since $h_{r}$ is the family of functional forms, this proposition implies that every functional form of this family is a flexible functional form. ${ }^{59}$

How do these two flexible functional forms $g_{r}$ and $h_{r}$ relate to each other? It is known that when the technology exhibits constant returns to scale, then (and only then) the output distance function is a reciprocal of the input distance function: ${ }^{60}$

$$
D_{o}(x, y)=\frac{1}{D_{i}(y, x)}
$$

It is worth noting that while we assume constant returns to scale technology, both $g_{r}$ and $h_{r}$ do not satisfy (68). In other words, when we assume $g_{r}$ for the output distance function, the corresponding input distance function has a functional form different from $h_{r}$. Similarly, when we assume $h_{r}$ for the input distance function, the corresponding output distance function has a functional form different from $g_{r}$. Therefore, it is inappropriate to assume $g_{r}$ for the output distance function and $h_{r}$ for the input distance function at the same time. ${ }^{61}$ Thus, we only assume either $g_{r}$ or $h_{r}$ but not both in the results that will be obtained in the next section. ${ }^{62}$

By dealing with the quadratic-mean-of-order- $r$ functional forms similar to one used in the present paper, Färe and Sung (1986) show that it belongs to the family of flexible functional forms called the generalized quadratic functional form. ${ }^{63}$ While they only deal with the function being linearly homogeneous in all variables, we deal with the function which is linearly homogeneous in all inputs and homogeneous of degree minus one in all outputs, or linearly homogeneous in all outputs and homogeneous of degree minus one in all inputs. Thus, while their result is only applicable to the distance functions with a single output or a single input, our results characterize the flexible functional form for the distance function in the more general case of multiple outputs and multiple inputs.

\footnotetext{
${ }^{58}$ Needless to say, $h_{r}$, defined by (60)-(62), is already a flexible functional form without imposing any restrictions (63)-(67).

${ }^{59}$ In the special case when $r=2$, Proposition 3 coincides with Theorem 5 of Diewert (1992), where $h_{2}$ was shown to be a flexible functional form.

${ }^{60}$ See Färe and Primont (1995).

${ }^{61}$ That is why we fail to derive the superlative index for the Hicks-Moorsteen productivity index from the superlative index results for output and input index, unlike for the case of the translog functional form. See Mizobuchi (2017) for more details.

62 Similar phenomena appears in Diewert (1992) for his functional forms.

${ }^{63}$ This functional form has an advantage of being linear in the parameters, which is convenient for estimation. However, both functional forms $g_{r}$ and $h_{r}$ defined by (52) and (60) do not belong to this family of functional form. Even if we transform $g_{r}$ and $h_{r}$ into $\left(g_{r}\right)^{r}$ and $\left(h_{r}\right)^{r}$, these functions $\left(g_{r}\right)^{r}$ and $\left(h_{r}\right)^{r}$ are not linear in the parameters. See Chambers (1988) for more on the generalized quadratic functional form.
} 


\section{Superlative indexes}

Here, we show that the quadratic-mean-of-order- $r$ indexes of output, input and productivity such as $Q M O I_{r}, Q M I I_{r}$ and $Q M P I_{r}$ coincide with the Malmquist indexes of output, input and productivity such as $M O I, M I I$ and $M P I$ under the assumption of optimizing behaviour as well as functional forms introduced in the previous section. This generalizes the results of Diewert (1992) obtained for the Fisher indexes.

\subsection{Output Index}

As is common in the literature (e.g., Caves et al. (1982), Diewert and Morrison (1986) and Diewert (1992)), we assume that a firm is engaged in revenue maximizing behaviour. Thus, outputs $y^{t}$ observed in period $t$ are considered as a solution to the following problem:

$$
\max _{y}\left\{p^{t} \cdot y \mid D_{o}^{t}\left(x^{t}, y\right) \leq 1\right\}
$$

Two implications can be drawn from it. First, the revenue maximization motive excludes inefficient use of inputs and outputs and induces production to take place on the output isoquant, which implies (7). Second, the above optimization problem implies the following first-order conditions:

$$
\frac{\partial D_{o}^{t}\left(x^{t}, y^{t}\right)}{\partial y_{m}}=\frac{p_{m}^{t}}{\sum_{j=1}^{M} p_{j}^{t} y_{j}^{t}}, \quad \forall m \in[1, \ldots, M]
$$

Equation (70) allows us to compute the derivatives of output distance functions without estimating its parameters. This information on the derivatives plays a key role in establishing the exact relationship between the quadratic-mean-of-order- $r$ and Malmquist output indexes, as stated by the next proposition.

We also assume that the period $t$ output distance function has the following functional form for $t=0,1$. This generalizes the functional form characterized in (52) by allowing technology to change over time. It is also defined for arbitrary non-zero real number $r$.

$$
\begin{aligned}
& g_{r}^{t}(y, x) \\
& \equiv \sigma^{t}\left[\left(\sum_{j=1}^{M} \sum_{k=1}^{M} a_{j, k} y_{j}^{r / 2} y_{k}^{r / 2}\right)\left(\sum_{u=1}^{N} \sum_{v=1}^{N} c_{u, v}^{t} x_{u}^{r / 2} x_{v}^{r / 2}\right)^{-1}\right. \\
& \left.+\left(\sum_{m=1}^{M} \alpha_{m}^{t} y_{m}^{r / 2}\right)\left(\sum_{n=1}^{N} \beta_{n}^{t} x_{n}^{-r / 2}\right)\left(\sum_{m=1}^{M} \sum_{n=1}^{N} b_{m, n}^{t} y_{m}^{r / 2} x_{n}^{-r / 2}\right)\right]^{1 / r}
\end{aligned}
$$

where

$$
\begin{aligned}
a_{j, k}=a_{k, j}, & \forall j, k \in[1, \ldots, M], \\
c_{u, v}^{t}=c_{v, u}^{t}, & \forall u, v \in[1, \ldots, N] .
\end{aligned}
$$


While a change in $\sigma^{t}$ affects the effective production of outputs and the effective use of inputs evenly, a change in other parameters such as $c_{u, v}^{t}, \alpha_{m}^{t}, \beta_{n}^{t}$ and $b_{m, n}^{t}$ has a different impact on inputs and outputs. Thus, the technical change we consider here is not limited to Hicks neutral and a variety of types of biased technical change are allowed.

Proposition 4: The quadratic-mean-of-order- $r$ output index defined by (34) is equal to the Malmquist output indexes defined in (26) and (30), so that $Q M O I_{r}=M O I^{0}=$ $M O I^{1}=M O I$, if the following two conditions are satisfied:

(1) the firm is engaged in revenue maximizing behaviour during periods 0 and 1 , so that $y^{t} \in \mathbb{R}_{++}^{M}$ is a solution to the optimization problem (69) for $t=0,1$;

(2) the period $t$ output distance function $D_{o}^{t}$ has the quadratic-mean-of-order- $r$ functional form $g_{r}^{t}$ as defined by (71)-(73), so that $D_{o}^{t}=g_{r}^{t}$ for $t=0,1$ and its parameters satisfy either the restrictions (74)-(75) or the restrictions (76) and (77):

$$
\begin{gathered}
\sum_{n=1}^{N} b_{m, n}^{0} x_{n}^{0^{-r / 2}}=0, \forall m \in[1, \ldots, M], \\
\sum_{n=1}^{N} \beta_{n}^{1} x_{n}^{1^{-r / 2}}=0 ; \\
\sum_{n=1}^{N} b_{m, n}^{1} x_{n}^{1-r / 2}=0, \forall m \in[1, \ldots, M], \\
\sum_{n=1}^{N} \beta_{n}^{0} x_{n}^{0^{-r / 2}}=0 .
\end{gathered}
$$

It shows that the two indexes coincide under several restrictions. Now, we examine their implications. As Proposition 2 shows, while the restrictions (74) and (75) allow $g_{r}^{0}$ to approximate an arbitrary output distance function to the second order at $\left(x^{0}, y^{0}\right)$, the restrictions (76) and (77) allow $g_{r}^{1}$ to approximate an arbitrary output distance function to the second order at $\left(x^{1}, y^{1}\right)$. Thus, according to Proposition 4 , the quadraticmean-of-order- $r$ and Malmquist output indexes can coincide when we assume the period 0 output distance function being flexible at $\left(x^{0}, y^{0}\right)$ or the period 1 output distance function being flexible at $\left(x^{1}, y^{1}\right)$. Moreover, since $Q M O I_{r}$ is a family of output indexes, this proposition also implies that every output index of this family is a superlative index. ${ }^{64}$

While $Q M O I_{r}$ is a formula of prices and quantities, the proof of the Proposition 4 shows that it can be transformed into a formula of quantities and parameters of the output distance function only. This transformation has an interesting implication for the axiomatic property of $Q M O I_{r}$, namely that it satisfies T21, as the following corollary shows:

Corollary 1 : The quadratic-mean-of-order- $r$ output index $Q M O I_{r}$ defined by (34) satisfied T21 (circularity test), if the conditions (1) and (2) of Proposition 4 are satisfied.

The conditions of Proposition 4 impose weaker restrictions on the underlying technology as well as the type of technical change. Thus, Corollary 1 implies that while

\footnotetext{
${ }^{64}$ In the special case when $r=2$, Proposition 4 coincides with Theorem 8 of Diewert (1992), where the Fisher output index was shown to be a superlative index.
} 
$Q M O I_{r}$ does not generally satisfy the circularity test, it could satisfy this test under some mild conditions. ${ }^{65}$

\subsection{Input Index}

Here, as in Diewert (1992), we assume that a firm is engaged in cost minimizing behaviour. Thus, inputs $x^{t}$ observed in period $t$ are considered as a solution to the following problem:

$$
\min _{x}\left\{w^{t} \cdot x \mid D_{i}^{t}\left(y^{t}, x\right) \geq 1\right\}
$$

Two implications can be drawn from it. First, the cost minimization motive excludes inefficient use of inputs and induces production to take place on the input isoquant, which implies (17). Second, the above optimization problem implies the following firstorder conditions:

$$
\frac{\partial D_{i}^{t}\left(y^{t}, x^{t}\right)}{\partial x_{n}}=\frac{w_{n}^{t}}{\sum_{u=1}^{N} w_{u}^{t} x_{u}^{t}}, \quad \forall n \in[1, \ldots, N]
$$

Thus, equation (79) allows us to compute the derivatives of input distance functions without estimating its parameters. This information on the derivatives plays a key role in establishing the exact relationship between the quadratic-mean-of-order- $r$ and Malmquist input indexes, as stated by the next proposition.

We also assume that the period $t$ input distance function has the following functional form for $t=0,1$. This generalizes the functional form characterized in (55) by allowing technology to change over time. It is also defined for arbitrary non-zero real number $r$.

$$
\begin{aligned}
& h_{r}^{t}(y, x) \\
& \equiv \sigma^{t}\left[\left(\sum_{j=1}^{M} \sum_{k=1}^{M} a_{j, k}^{t} y_{j}^{r / 2} y_{k}^{r / 2}\right)^{-1}\left(\sum_{u=1}^{N} \sum_{v=1}^{N} c_{u, v} x_{u}^{r / 2} x_{v}^{r / 2}\right)\right. \\
& \left.+\left(\sum_{m=1}^{M} \alpha_{m}^{t} y_{m}^{-r / 2}\right)\left(\sum_{n=1}^{N} \beta_{n}^{t} x_{n}^{r / 2}\right)\left(\sum_{m=1}^{M} \sum_{n=1}^{N} b_{m, n}^{t} y_{m}^{-r / 2} x_{n}^{r / 2}\right)\right]^{1 / r}
\end{aligned}
$$

where

$$
\begin{array}{ll}
a_{j, k}^{t}=a_{k, j}^{t}, & \forall j, k \in[1, \ldots, M], \\
c_{u, v}=c_{v, u}, & \forall u, v \in[1, \ldots, N] .
\end{array}
$$

\footnotetext{
${ }^{65}$ Obviously, Proposition 4 and Corollary 1 imply that the Malmquist output index also satisfies the circularity test.
} 
Just like the case of $g_{r}^{t}$, a variety of parameters of $h_{r}^{t}$ are allowed to vary across time. Thus, the technical change we consider is not limited to Hicks neutral and a variety of types of biased technical change are allowed.

Proposition 5: The quadratic-mean-of-order- $r$ input index defined by (40) is equal to the Malmquist input indexes defined in (27) and (31), so that $Q M I I_{r}=M I I^{0}=M I I^{1}=$ $M I I$, if the following two conditions are satisfied:

(1) the firm is engaged in cost minimizing behaviour during periods 0 and 1 , so that $x^{t} \in \mathbb{R}_{++}^{N}$ is a solution to the optimization problem (78) for $t=0,1$;

(2) the period $t$ input distance function $D_{i}^{t}$ has the quadratic-mean-of-order- $r$ functional form $h_{r}^{t}$ as defined by (80)-(82), so that $D_{i}^{t}=h_{r}^{t}$ for $t=0,1$, and its parameters satisfy either the restrictions (83)-(84) or the restrictions (85) and (86):

$$
\begin{gathered}
\sum_{m=1}^{M} b_{m, n}^{0} y_{m}^{0}{ }^{-r / 2}=0, \forall n \in[1, \ldots, N], \\
\sum_{m=1}^{M} \alpha_{m}^{1} y_{m}^{1}-r / 2=0 ; \\
\sum_{m=1}^{M} b_{m, n}^{1} y_{m}^{1}{ }^{-r / 2}=0, \forall n \in[1, \ldots, N], \\
\sum_{m=1}^{M} \alpha_{m}^{0} y_{m}^{0-r / 2}=0 .
\end{gathered}
$$

It shows that the two indexes coincide under several restrictions. Now, we examine their implications. As Proposition 3 shows, while the restrictions (83) and (84) allow $h_{r}^{0}$ to approximate an arbitrary input distance function to the second order at $\left(x^{0}, y^{0}\right)$, the restrictions (85) and (86) allow $h_{r}^{1}$ to approximate an arbitrary input distance function to the second order at $\left(x^{1}, y^{1}\right)$. Thus, according to Proposition 5, the quadraticmean-of-order- $r$ and Malmquist input indexes can coincide when we assume the period 0 input distance function being flexible at $\left(x^{0}, y^{0}\right)$ or the period 1 input distance function being flexible at $\left(x^{1}, y^{1}\right)$. Moreover, since $Q M I I_{r}$ is a family of input indexes, this proposition also implies that every input index of this family is a superlative index. ${ }^{66}$

The proof of Proposition 5 allow us to draw an implication of the axiomatic property that $Q M I I_{r}$ satisfies, as the following corollary shows:

Corollary 2 : The quadratic-mean-of-order- $r$ input index $Q M I I_{r}$ defined by (40) satisfied T21 (circularity test), if the conditions (1) and (2) of Proposition 5 are satisfied.

As Corollary 1 claims for $Q M O I_{r}$, Corollary 2 also implies that $Q M I I_{r}$ could satisfy the circularity test under some mild conditions. ${ }^{67}$

\footnotetext{
${ }^{66}$ In the special case when $r=2$, Proposition 5 coincides with Theorem 6 of Diewert (1992), where the Fisher input index was shown to be a superlative index.

${ }^{67}$ Note that Proposition 5 and Corollary 2 imply that the Malmquist input index also satisfies the circularity test.
} 


\subsection{Productivity Index}

Again, following the literature, we assume that a firm is engaged in profit maximizing behaviour, which implies revenue maximizing and cost minimizing behaviour at the same time. Thus, the production plan $\left(x^{t}, y^{t}\right)$ observed in period $t$ is considered as a solution to the following problem: ${ }^{68}$

$$
\max _{x, y}\left\{p^{t} \cdot y-w^{t} \cdot x \mid D_{o}^{t}(x, y) \leq 1\right\}
$$

Two implications can be drawn from it. First, the revenue maximization motive excludes inefficient use of inputs and outputs and induces production to take place on the production frontier, which implies (7). Second, the above optimization problem implies the following first-order conditions:

$$
\begin{gathered}
\frac{\partial D_{o}^{t}\left(x^{t}, y^{t}\right)}{\partial y_{m}}=\frac{p_{m}^{t}}{\sum_{j=1}^{M} p_{j}^{t} y_{j}^{t}}, \quad \forall m \in[1, \ldots, M], \\
\frac{\partial D_{o}^{t}\left(x^{t}, y^{t}\right)}{\partial x_{n}}=-w_{n}^{t} / \sum_{m=1}^{M} p_{m}^{t} y_{m}^{t}, \quad \forall n \in[1, \ldots, N] .
\end{gathered}
$$

Along with (10) and (13), they lead to the following zero economic profit condition:

$$
\sum_{m=1}^{M} p_{m}^{t} y_{m}^{t}=\sum_{n=1}^{N} w_{n}^{t} x_{n}^{t}
$$

Equations (88) and (89) allow us to compute the derivatives of output distance function without estimating its parameters. This information on the derivatives plays a key role in establishing the exact relationship between the quadratic-mean-of-order- $r$ and Malmquist productivity indexes, as stated by the next proposition.

We also assume that the period $t$ output distance function has the following functional form for $t=0,1$. This generalizes the functional form characterized in (52) by allowing technology to change over time. It is also defined for arbitrary non-zero real number $r$.

\footnotetext{
${ }^{68}$ While the profit maximization problem (87) is formulated by the output distance function, it is possible to define it by the input distance function.
} 


$$
\begin{aligned}
& \hat{g}_{r}^{t}(x, y) \\
& \equiv \sigma^{t}\left[\left(\sum_{j=1}^{M} \sum_{k=1}^{M} a_{j, k} y_{j}^{r / 2} y_{k}^{r / 2}\right)\left(\sum_{u=1}^{N} \sum_{v=1}^{N} c_{u, v} x_{u}^{r / 2} x_{v}^{r / 2}\right)^{-1}\right. \\
& \left.+\left(\sum_{m=1}^{M} \alpha_{m}^{t} y_{m}^{r / 2}\right)\left(\sum_{n=1}^{N} \beta_{n}^{t} x_{n}^{-r / 2}\right)\left(\sum_{m=1}^{M} \sum_{n=1}^{N} b_{m, n}^{t} y_{m}^{r / 2} x_{n}^{-r / 2}\right)\right]^{1 / r}
\end{aligned}
$$

where

$$
\begin{aligned}
a_{j, k}=a_{k, j}, & \forall j, k \in[1, \ldots, M], \\
c_{u, v}=c_{v, u}, & \forall u, v \in[1, \ldots, N] .
\end{aligned}
$$

Just like the case of $g_{r}^{t}$ and $h_{r}^{t}$, a variety of parameters of $\hat{g}_{r}^{t}$ are allowed to vary across time. Thus, the technical change we consider is not limited to Hicks neutral and a variety of types of biased technical change are allowed. ${ }^{69}$

Proposition 6: The quadratic-mean-of-order- $r$ productivity index defined by (46) is equal to the Malmquist productivity indexes defined in (28) and (32), so that $Q M P I_{r}=$ $M P I^{0}=M P I^{1}=M P I$, if the following two conditions are satisfied:

(1) the firm is engaged in profit maximizing behaviour during periods 0 and 1 , so that $\left(x^{t}, y^{t}\right) \in \mathbb{R}_{++}^{N+M}$ is a solution to the optimization problem (87) for $t=0,1$;

(2) the period $t$ output distance function $D_{o}^{t}$ has the quadratic-mean-of-order- $r$ functional form $\hat{g}_{r}^{t}$ as defined by (91)-(93), so that $D_{o}^{t}=\hat{g}_{r}^{t}$ for $t=0,1$, and its parameters satisfy either the restrictions (94)-(99) or the restrictions (100)-(105):

$$
\begin{gathered}
\sum_{n=1}^{N} b_{m, n}^{0} x_{n}^{0^{-r / 2}}=0, \forall m \in[1, \ldots, M], \\
\sum_{m=1}^{M} b_{m, n}^{0} y_{m}^{0}{ }^{r / 2}=0, \forall n \in[1, \ldots, N], \\
\sum_{m=1}^{M} \alpha_{m}^{0} y_{m}^{1}{ }^{r / 2} \sum_{n=1}^{N} \beta_{n}^{0} x_{n}^{1}{ }^{-r / 2}=0, \\
\sum_{m=1}^{M} \alpha_{m}^{1} y_{m}^{1}{ }^{r / 2}=0, \\
\sum_{n=1}^{N} \beta_{n}^{1} x_{n}^{1^{-r / 2}}=0, \\
\sum_{m=1}^{M} \sum_{n=1}^{N} b_{m, n}^{1} y_{n}^{0^{r / 2}} x_{n}^{0^{-r / 2}}=0 ; \\
\sum_{n=1}^{N} b_{m, n}^{1} x_{n}^{1}{ }^{-r / 2}=0, \forall m \in[1, \ldots, M], \\
\sum_{m=1}^{M} b_{m, n}^{1} y_{m}^{1}{ }^{r / 2}=0, \forall n \in[1, \ldots, N], \\
\sum_{m=1}^{M} \alpha_{m}^{1} y_{m}^{0}{ }^{r / 2} \sum_{n=1}^{N} \beta_{n}^{1} x_{n}^{0-r / 2}=0, \\
\sum_{m=1}^{M} \alpha_{m}^{0} y_{m}^{{ }^{r / 2}}=0, \\
\sum_{n=1}^{N} \beta_{n}^{0} x_{n}^{0^{-r / 2}}=0, \\
\sum_{m=1}^{M} \sum_{n=1}^{N} b_{m, n}^{0} y_{n}^{1}{ }^{r / 2} x_{n}^{1}{ }^{-r / 2}=0 .
\end{gathered}
$$

\footnotetext{
${ }^{69}$ Unlike the case of $g_{r}^{t}$, a parameter $c_{u, v}$ is fixed. However, a change in other parameters such as $\alpha_{m}^{t}$, $\beta_{n}^{t}$ and $b_{m, n}^{t}$ has a different impact on inputs and outputs. Thus, it is possible to deal with the biased technical change under a functional form $\hat{g}_{r}^{t}$.
} 
It shows that the two indexes coincide under several restrictions. Now, we examine the implications of the first set of restrictions (94)-(99). As Proposition 2 shows, the restrictions (94)-(96) allow $\hat{g}_{r}^{0}$ to approximate an arbitrary output distance function to the second order at $\left(x^{0}, y^{0}\right)$. The remaining restrictions (97)-(99) are imposed on the parameters of $\hat{g}_{r}^{1} .{ }^{70} \mathrm{Next}$, we examine the implications of the second set of restrictions (100)-(105). As Proposition 2 shows, the restrictions (100)-(102) allow $\hat{g}_{r}^{1}$ to approximate an arbitrary output distance function to the second order at $\left(x^{1}, y^{1}\right)$. The remaining restrictions (103)-(105) are imposed on the parameters of $\hat{g}_{r}^{0} \cdot{ }^{71}$ Therefore, there is a possibility that these remaining restrictions (97)-(99) and (103)-(105) might limit the way the underlying technology changes. ${ }^{72}$ However, it is worth noting that these remaining restrictions (97)-(99) and (103)-(105) disappear under Hicks neutral technical change. It means that $\hat{g}_{r}^{t}$ is allowed to capture some types of bias associated with technical change, even under these remaining restrictions.

Thus, as Propositions 4 and 5 show for $Q M O I_{r}$ and $Q M I I_{r}$, Proposition 6 also indicates that even in the existence of biased technical change, the quadratic-mean-of-order- $r$ and Malmquist productivity indexes can coincide when we assume the period 0 output distance function being flexible at $\left(x^{0}, y^{0}\right)$ or the period 1 output distance function being flexible at $\left(x^{1}, y^{1}\right)$. Moreover, since $Q M P I_{r}$ is a family of productivity indexes, this proportion also implies that every productivity index of this family is a superlative index. $^{73}$

The proof of Proposition 6 allows us to draw an implication of the axiomatic property that $Q M P I_{r}$ satisfies, as the following corollary shows:

Corollary 3 : The quadratic-mean-of-order- $r$ output index $Q M P I_{r}$ defined by (46) satisfied T21 (circularity test), if the conditions (1) and (2) of Proposition 6 are satisfied:

As Corollaries 1 and 2 claim for $Q M O I_{r}$ and $Q M I I_{r}$, Corollary 3 also implies that $Q M P I_{r}$ could satisfy the circularity test under some mild conditions. ${ }^{74}$

\footnotetext{
${ }^{70}$ As Proposition 2 shows, since $\alpha_{m}^{1}$ and $\beta_{n}^{1}$ for all $m$ and $n$ can be freely chosen without destroying the flexibility of $\hat{g}_{r}^{1}$, the restrictions (97) and (98) are harmless.

${ }^{71}$ As Proposition 2 shows, since $\alpha_{m}^{0}$ and $\beta_{n}^{0}$ for all $m$ and $n$ can be freely chosen without destroying the flexibility of $\hat{g}_{r}^{0}$, the restrictions (103) and (104) are harmless.

72 As mentioned in the previous footnotes, the restrictions (97), (98), (103) and (104) do not lose the flexibility for $\hat{g}_{r}^{0}$ and $\hat{g}_{r}^{1}$ to represent the underlying technology. Thus, only the restrictions (99) and (105) may matter.

${ }^{73}$ In the special case when $r=2$, Proposition 6 coincides with Theorem 9 of Diewert (1992), where the Fisher productivity index was shown to be a superlative index.

${ }^{74}$ Note that Proposition 6 and Corollary 3 imply that the Malmquist productivity index also satisfies the circularity test. Thus, while Färe and Grosskopf (1996) and Sickles and Zelenyuk (2019) point out that the Malmquist productivity index satisfies the circularity test under Hicks neutral technical change, we show that the Malmquist productivity index could satisfy the circularity test even under biased technical change.
} 


\section{Empirical Application}

In the previous sections, we showed that the quadratic-mean-of-order- $r$ indexes of output, input and productivity are superlative indexes. Since each quadratic-mean-oforder- $r$ index is a family of indexes, our results are interpreted as showing that all indexes that belong to these families are superlative indexes. ${ }^{75}$ This justifies an infinite number of empirical index numbers. Naturally, it raises the question of which index one should use.

Importantly, Diewert (1978) shows that all quadratic-mean-of-order- $r$ price (quantity) indexes approximate each other to the second order around the point that price and quantity vectors in two periods coincide. It indicates that when the changes in prices and quantities are small enough, the difference among indexes in the family of the quadratic-mean-of-order- $r$ output (input) indexes also becomes small. On the other hand, by using datasets for the US and OECD countries, Hill (2006) shows that the spread between quadratic-mean-of-order- $r$ price indexes would be significant and the quadratic-mean-of-order- $r$ price index could exceed the spread between Laspeyres and Paasche indexes, when the parameter $r$ increases in absolute terms.

Since Diewert (1978) and Hill (2006) deal with price and quantity indexes, their analysis is only applicable to the quadratic-mean-of-order- $r$ output and input indexes. However, to the best of our knowledge, no study so far has implemented the quadraticmean-of-order- $r$ productivity indexes under different values of $r$. In this section, we fill this gap in the literature by empirically comparing the quadratic mean of order $r$ indexes of output, input and productivity with other index number formulae by using the most recent integrated industry-level production accounts for the US economy. It has been published by the key authorities on US data: the Bureau of Economic Analysis (BEA) and the Bureau of Labor Statistics (BLS). This database consists of price and quantities of output and input for 63 detailed industries during the period 1998-2017. It has the advantage of being constructed in a manner consistent with the National Income and Product Accounts (NIPAs).

While aggregating the inputs and outputs for 63 industries, we can measure the aggregate output and input change and the aggregate productivity growth. In this exercise, since we presume that each industry produces a single output (industry value added) from seven inputs (five types of capital and two types of labour), the output index aggregates change in quantities of 63 outputs and the input index aggregates change in quantities of 441 inputs in this particular case. ${ }^{76}$

\footnotetext{
75 To be more precise, we deal with three families of indexes: the quadratic-mean-of-order-r indexes of output, of input and of productivity.

${ }^{76}$ Five types of capital input include: IT Capital, R\&D Capital, Software Capital, Entertainment Originals Capital, Other Capital. The two types of labour input include College Labour, Non-College Labour There are also three types of intermediate inputs: Energy, Materials and Purchased-services. Prices of distinct input vary across industries by reflecting the difference in its composition. Thus, we consider that each industry uses unique and different seven inputs. Thus, for example, IT capital for the machinery industry is regarded as different from that for the wholesale trade industry.
} 
Table 2: Quadratic-mean-of-order-r output index

\begin{tabular}{|c|c|c|c|c|c|c|c|c|c|c|c|c|c|c|c|}
\hline & \multicolumn{11}{|c|}{ Quadratic-mean-of-order- $r$} & \multirow[t]{2}{*}{ Laspeyres } & \multirow[t]{2}{*}{ Paasche } & \multirow[t]{2}{*}{ Törnqvist } & \multirow[t]{2}{*}{ Walsh } \\
\hline & $r=0.5$ & $r=1$ & $r=1.5$ & $r=2$ & $r=2.5$ & $r=3$ & $r=5$ & $r=10$ & $r=20$ & $r=50$ & $r=100$ & & & & \\
\hline \multicolumn{16}{|c|}{ annual growth rate (\%) } \\
\hline 2002 & 2.03 & 2.03 & 2.03 & 2.03 & 2.03 & 2.03 & 2.04 & 2.06 & 2.15 & 0.18 & -1.21 & 2.05 & 2.01 & 1.87 & 2.03 \\
\hline 2003 & 2.76 & 2.76 & 2.76 & 2.76 & 2.76 & 2.76 & 2.76 & 2.79 & 2.95 & 4.23 & 4.71 & 2.83 & 2.69 & 2.68 & 2.76 \\
\hline 2004 & 3.71 & 3.71 & 3.71 & 3.71 & 3.71 & 3.71 & 3.71 & 3.72 & 3.74 & 3.88 & 4.11 & 3.75 & 3.66 & 3.64 & 3.71 \\
\hline 2005 & 3.46 & 3.46 & 3.46 & 3.46 & 3.46 & 3.46 & 3.47 & 3.48 & 3.53 & 3.79 & 3.64 & 3.53 & 3.40 & 3.43 & 3.46 \\
\hline 2006 & 2.90 & 2.90 & 2.90 & 2.90 & 2.90 & 2.91 & 2.92 & 2.98 & 3.39 & 9.80 & 14.57 & 2.96 & 2.84 & 2.83 & 2.90 \\
\hline 2007 & 1.68 & 1.68 & 1.68 & 1.68 & 1.68 & 1.68 & 1.68 & 1.65 & 1.49 & 0.22 & -0.52 & 1.75 & 1.62 & 1.57 & 1.68 \\
\hline 2008 & -0.22 & -0.21 & -0.20 & -0.20 & -0.21 & -0.23 & -0.36 & -1.53 & -9.26 & -20.03 & -21.19 & -0.19 & -0.22 & -0.65 & -0.20 \\
\hline 2009 & -2.52 & -2.52 & -2.52 & -2.50 & -2.48 & -2.45 & -2.22 & -0.34 & 5.11 & 3.85 & 3.20 & -2.15 & -2.86 & -2.96 & -2.54 \\
\hline 2010 & 2.36 & 2.36 & 2.36 & 2.36 & 2.36 & 2.36 & 2.41 & 2.84 & 7.77 & 21.21 & 23.56 & 2.47 & 2.24 & 2.23 & 2.36 \\
\hline 2011 & 1.46 & 1.46 & 1.46 & 1.46 & 1.46 & 1.46 & 1.45 & 1.40 & 1.19 & -0.15 & -0.32 & 1.54 & 1.38 & 1.40 & 1.46 \\
\hline 2012 & 2.10 & 2.10 & 2.10 & 2.10 & 2.10 & 2.10 & 2.11 & 2.15 & 2.28 & 0.61 & -2.92 & 2.15 & 2.06 & 2.03 & 2.10 \\
\hline 2013 & 1.56 & 1.56 & 1.56 & 1.56 & 1.56 & 1.56 & 1.57 & 1.60 & 1.75 & 3.32 & 5.93 & 1.58 & 1.53 & 1.50 & 1.56 \\
\hline 2014 & 2.19 & 2.19 & 2.19 & 2.19 & 2.19 & 2.19 & 2.20 & 2.26 & 3.09 & 14.12 & 17.96 & 2.23 & 2.15 & 2.10 & 2.19 \\
\hline 2015 & 2.75 & 2.76 & 2.76 & 2.76 & 2.76 & 2.77 & 2.77 & 2.76 & 2.34 & -3.17 & -5.35 & 2.92 & 2.61 & 2.74 & 2.74 \\
\hline 2016 & 1.60 & 1.60 & 1.60 & 1.60 & 1.60 & 1.60 & 1.58 & 1.42 & -0.55 & -12.53 & -15.62 & 1.63 & 1.57 & 1.50 & 1.61 \\
\hline 2017 & 1.81 & 1.81 & 1.81 & 1.81 & 1.81 & 1.81 & 1.82 & 1.82 & 1.85 & 2.09 & 2.85 & 1.85 & 1.78 & 1.80 & 1.82 \\
\hline average & 1.85 & 1.85 & 1.85 & 1.86 & 1.86 & 1.86 & 1.87 & 1.94 & 2.05 & 1.96 & 2.09 & 1.93 & 1.78 & 1.73 & 1.85 \\
\hline \multicolumn{16}{|c|}{ cumulative growth rate (\%) } \\
\hline 2002-2017 & 29.65 & 29.66 & 29.67 & 29.69 & 29.71 & 29.73 & 29.88 & 31.05 & 32.82 & 31.42 & 33.42 & 30.91 & 28.47 & 27.72 & 29.65 \\
\hline $2002-2007$ & 16.55 & 16.55 & 16.55 & 16.55 & 16.55 & 16.55 & 16.57 & 16.67 & 17.26 & 22.09 & 25.31 & 16.87 & 16.23 & 16.02 & 16.55 \\
\hline 2007-2009 & 13.11 & 13.11 & 13.12 & 13.14 & 13.16 & 13.18 & 13.31 & 14.38 & 15.56 & 9.33 & 8.11 & 14.04 & 12.24 & 11.70 & 13.09 \\
\hline 2009-2017 & 13.33 & 13.32 & 13.33 & 13.34 & 13.37 & 13.41 & 13.68 & 15.91 & 24.82 & 29.36 & 29.30 & 14.23 & 12.46 & 12.35 & 13.30 \\
\hline
\end{tabular}


Table 3: Quadratic-mean-of-order-r input index

\begin{tabular}{|c|c|c|c|c|c|c|c|c|c|c|c|c|c|c|c|}
\hline & \multicolumn{11}{|c|}{ Quadratic-mean-of-order- $r$} & \multirow[t]{2}{*}{ Laspeyres } & \multirow[t]{2}{*}{ Paasche } & \multirow[t]{2}{*}{ Törnqvist } & \multirow[t]{2}{*}{ Walsh } \\
\hline & $r=0.5$ & $r=1$ & $r=1.5$ & $r=2$ & $r=2.5$ & $r=3$ & $r=5$ & $r=10$ & $r=20$ & $r=50$ & $r=100$ & & & & \\
\hline \multicolumn{16}{|c|}{ annual growth rate (\%) } \\
\hline 2002 & 0.96 & 0.96 & 0.96 & 0.96 & 0.96 & 0.96 & 0.96 & 0.98 & 1.03 & 1.89 & 3.73 & 0.97 & 0.95 & 0.81 & 0.95 \\
\hline 2003 & 1.44 & 1.44 & 1.44 & 1.44 & 1.44 & 1.44 & 1.45 & 1.45 & 1.46 & 1.56 & 1.41 & 1.47 & 1.41 & 1.33 & 1.44 \\
\hline 2004 & 2.27 & 2.27 & 2.27 & 2.27 & 2.27 & 2.27 & 2.28 & 2.29 & 2.37 & 2.38 & 0.03 & 2.29 & 2.25 & 2.20 & 2.27 \\
\hline 2005 & 2.21 & 2.21 & 2.22 & 2.22 & 2.22 & 2.22 & 2.22 & 2.25 & 2.43 & 7.67 & 10.29 & 2.24 & 2.19 & 2.17 & 2.21 \\
\hline 2006 & 2.88 & 2.88 & 2.88 & 2.88 & 2.88 & 2.88 & 2.89 & 2.91 & 3.03 & 5.56 & 8.53 & 2.92 & 2.84 & 2.84 & 2.88 \\
\hline 2007 & 1.96 & 1.96 & 1.96 & 1.96 & 1.96 & 1.96 & 1.96 & 1.99 & 2.11 & 5.77 & 11.51 & 2.01 & 1.91 & 1.91 & 1.96 \\
\hline 2008 & 0.98 & 0.98 & 0.98 & 0.98 & 0.98 & 0.98 & 0.98 & 0.98 & 0.98 & 1.76 & 9.73 & 1.00 & 0.96 & 0.89 & 0.98 \\
\hline 2009 & -2.02 & -2.02 & -2.02 & -2.02 & -2.02 & -2.02 & -2.04 & -2.11 & -2.43 & 5.42 & 13.01 & -1.93 & -2.12 & -2.14 & -2.02 \\
\hline 2010 & 0.59 & 0.58 & 0.58 & 0.58 & 0.58 & 0.58 & 0.57 & 0.48 & -0.66 & -4.53 & 0.21 & 0.62 & 0.55 & 0.54 & 0.59 \\
\hline 2011 & 1.76 & 1.76 & 1.76 & 1.76 & 1.76 & 1.76 & 1.77 & 1.79 & 1.90 & 8.10 & 18.20 & 1.78 & 1.74 & 1.70 & 1.76 \\
\hline 2012 & 1.76 & 1.76 & 1.77 & 1.77 & 1.77 & 1.77 & 1.77 & 1.79 & 1.89 & 7.78 & 18.25 & 1.79 & 1.74 & 1.73 & 1.76 \\
\hline 2013 & 1.79 & 1.79 & 1.79 & 1.79 & 1.79 & 1.79 & 1.79 & 1.81 & 1.87 & 2.65 & 6.41 & 1.81 & 1.78 & 1.76 & 1.79 \\
\hline 2014 & 1.85 & 1.85 & 1.85 & 1.85 & 1.86 & 1.86 & 1.86 & 1.88 & 2.00 & 5.36 & 14.53 & 1.86 & 1.85 & 1.81 & 1.85 \\
\hline 2015 & 2.27 & 2.27 & 2.27 & 2.27 & 2.27 & 2.27 & 2.27 & 2.27 & 2.27 & 1.59 & -0.80 & 2.26 & 2.27 & 2.20 & 2.27 \\
\hline 2016 & 2.13 & 2.13 & 2.13 & 2.13 & 2.13 & 2.13 & 2.13 & 2.13 & 2.07 & -0.38 & -0.66 & 2.13 & 2.14 & 2.05 & 2.13 \\
\hline 2017 & 1.52 & 1.52 & 1.52 & 1.52 & 1.52 & 1.52 & 1.52 & 1.53 & 1.52 & 0.17 & -3.47 & 1.55 & 1.50 & 1.48 & 1.52 \\
\hline average & 1.52 & 1.52 & 1.52 & 1.52 & 1.52 & 1.52 & 1.52 & 1.53 & 1.49 & 3.30 & 6.93 & 1.55 & 1.50 & 1.46 & 1.52 \\
\hline \multicolumn{16}{|c|}{ cumulative growth rate (\%) } \\
\hline 2002-2017 & 24.36 & 24.36 & 24.36 & 24.37 & 24.37 & 24.37 & 24.38 & 24.40 & 23.85 & 52.75 & 110.93 & 24.78 & 23.95 & 23.29 & 24.35 \\
\hline $2002-2007$ & 11.73 & 11.73 & 11.73 & 11.73 & 11.73 & 11.74 & 11.76 & 11.87 & 12.45 & 24.82 & 35.51 & 11.91 & 11.55 & 11.28 & 11.71 \\
\hline 2007-2009 & 12.63 & 12.63 & 12.64 & 12.64 & 12.63 & 12.63 & 12.62 & 12.53 & 11.40 & 27.93 & 75.42 & 12.87 & 12.40 & 12.02 & 12.64 \\
\hline 2009-2017 & 11.66 & 11.66 & 11.66 & 11.66 & 11.66 & 11.66 & 11.64 & 11.55 & 10.42 & 26.17 & 65.69 & 11.88 & 11.44 & 11.12 & 11.66 \\
\hline
\end{tabular}


Table 4: Quadratic-mean-of-order-r productivity index

\begin{tabular}{|c|c|c|c|c|c|c|c|c|c|c|c|c|c|c|c|}
\hline & \multicolumn{11}{|c|}{ Quadratic-mean-of-order- $r$} & \multirow[t]{2}{*}{ Laspeyres } & \multirow[t]{2}{*}{ Paasche } & \multirow[t]{2}{*}{ Törnqvist } & \multirow[t]{2}{*}{ Walsh } \\
\hline & $r=0.5$ & $r=1$ & $r=1.5$ & $r=2$ & $r=2.5$ & $r=3$ & $r=5$ & $r=10$ & $r=20$ & $r=50$ & $r=100$ & & & & \\
\hline \multicolumn{16}{|c|}{ annual growth rate (\%) } \\
\hline 2002 & 1.06 & 1.06 & 1.06 & 1.06 & 1.06 & 1.06 & 1.06 & 1.07 & 1.10 & -1.68 & -4.76 & 1.07 & 1.05 & 1.05 & 1.07 \\
\hline 2003 & 1.30 & 1.30 & 1.30 & 1.30 & 1.30 & 1.30 & 1.30 & 1.32 & 1.47 & 2.63 & 3.26 & 1.33 & 1.26 & 1.33 & 1.30 \\
\hline 2004 & 1.40 & 1.40 & 1.40 & 1.41 & 1.41 & 1.40 & 1.40 & 1.39 & 1.34 & 1.46 & 4.07 & 1.43 & 1.38 & 1.41 & 1.40 \\
\hline 2005 & 1.22 & 1.22 & 1.22 & 1.22 & 1.22 & 1.22 & 1.22 & 1.20 & 1.08 & -3.61 & -6.03 & 1.25 & 1.19 & 1.23 & 1.22 \\
\hline 2006 & 0.02 & 0.02 & 0.02 & 0.02 & 0.02 & 0.02 & 0.03 & 0.07 & 0.35 & 4.02 & 5.56 & 0.04 & 0.00 & -0.01 & 0.02 \\
\hline 2007 & -0.27 & -0.27 & -0.27 & -0.27 & -0.27 & -0.27 & -0.28 & -0.33 & -0.61 & -5.24 & -10.79 & -0.25 & -0.28 & -0.34 & -0.27 \\
\hline 2008 & -1.19 & -1.18 & -1.17 & -1.17 & -1.18 & -1.19 & -1.33 & -2.48 & -10.15 & -21.42 & -28.17 & -1.17 & -1.17 & -1.53 & -1.17 \\
\hline 2009 & -0.51 & -0.51 & -0.51 & -0.49 & -0.47 & -0.44 & -0.19 & 1.81 & 7.73 & -1.49 & -8.68 & -0.22 & -0.76 & -0.84 & -0.53 \\
\hline 2010 & 1.77 & 1.76 & 1.76 & 1.76 & 1.77 & 1.77 & 1.83 & 2.35 & 8.49 & 26.96 & 23.30 & 1.84 & 1.68 & 1.68 & 1.76 \\
\hline 2011 & -0.29 & -0.29 & -0.29 & -0.30 & -0.30 & -0.30 & -0.31 & -0.38 & -0.70 & -7.63 & -15.67 & -0.24 & -0.35 & -0.29 & -0.29 \\
\hline 2012 & 0.33 & 0.33 & 0.33 & 0.33 & 0.33 & 0.33 & 0.33 & 0.35 & 0.38 & -6.65 & -17.90 & 0.35 & 0.31 & 0.29 & 0.33 \\
\hline 2013 & -0.23 & -0.23 & -0.23 & -0.23 & -0.23 & -0.23 & -0.23 & -0.21 & -0.12 & 0.66 & -0.46 & -0.22 & -0.24 & -0.25 & -0.23 \\
\hline 2014 & 0.33 & 0.33 & 0.33 & 0.33 & 0.33 & 0.33 & 0.34 & 0.38 & 1.07 & 8.32 & 3.00 & 0.37 & 0.30 & 0.29 & 0.33 \\
\hline 2015 & 0.47 & 0.48 & 0.48 & 0.48 & 0.48 & 0.49 & 0.49 & 0.48 & 0.07 & -4.69 & -4.59 & 0.64 & 0.33 & 0.53 & 0.46 \\
\hline 2016 & -0.52 & -0.52 & -0.52 & -0.52 & -0.52 & -0.52 & -0.54 & -0.70 & -2.57 & -12.19 & -15.06 & -0.49 & -0.55 & -0.54 & -0.52 \\
\hline 2017 & 0.29 & 0.29 & 0.29 & 0.29 & 0.29 & 0.29 & 0.29 & 0.29 & 0.32 & 1.91 & 6.55 & 0.30 & 0.28 & 0.32 & 0.29 \\
\hline average & 0.32 & 0.33 & 0.33 & 0.33 & 0.33 & 0.33 & 0.34 & 0.41 & 0.58 & -1.16 & -4.15 & 0.38 & 0.28 & 0.27 & 0.32 \\
\hline \multicolumn{16}{|c|}{ cumulative growth rate (\%) } \\
\hline 2002-2017 & 5.20 & 5.20 & 5.21 & 5.22 & 5.24 & 5.26 & 5.41 & 6.62 & 9.26 & -18.64 & -66.37 & 6.02 & 4.42 & 4.33 & 5.19 \\
\hline $2002-2007$ & 4.74 & 4.74 & 4.74 & 4.73 & 4.73 & 4.73 & 4.73 & 4.72 & 4.72 & -2.42 & -8.69 & 4.88 & 4.59 & 4.67 & 4.75 \\
\hline 2007-2009 & 0.46 & 0.46 & 0.47 & 0.49 & 0.51 & 0.53 & 0.68 & 1.90 & 4.53 & -16.22 & -57.68 & 1.15 & -0.17 & -0.34 & 0.44 \\
\hline 2009-2017 & 1.64 & 1.64 & 1.64 & 1.66 & 1.69 & 1.72 & 2.01 & 4.38 & 14.68 & 5.19 & -29.51 & 2.32 & 1.00 & 1.19 & 1.61 \\
\hline
\end{tabular}


Table 2, Table 3Table 4 show the annual growth rate of output and input computed by different quantity indexes. ${ }^{77}$ It turns out that for this particular data set, all quadraticmean-of-order $r$ indexes are almost the same for the range of $r$ from 0.5 to 5 . They are also very close to the Walsh indexes as well. Thus, the popular indexes of Fisher $(r=$ 2 ), implicit Walsh $(r=1)$ and the Walsh index almost coincide with each other. ${ }^{78}$ This is true for the indexes of output, input and productivity.

While Hill (2006) theoretically showed that the quadratic-mean-of-order- $r$ quantity index never exceeds the spread between the Paasche and Laspeyres indexes only for the case $r=2$, the quadratic-mean-of-order- $r$ output and input indexes are found to lie between the Paasche and Laspeyres indexes for a wider range of $r$ for this particular example: the range from 1 to 2.5 for output index and the range from 0.5 to 5 for the input index. ${ }^{79}$ Meanwhile, the productivity indexes considered here are defined as the ratios of output indexes to corresponding input indexes and thus are subject to the fluctuation of both output and input indexes. Thus, the quadratic-mean-of-order- $r$ productivity index lies between the Paasche and Laspeyres indexes for the range of $r$ from 1.5 to 2 , which is considerably smaller than the range of $r$ where the quadraticmean-of-order- $r$ output and input indexes lie between those indexes. ${ }^{80}$

However, when $r$ gets large enough, the quadratic-mean-of-order- $r$ index would largely exceed the spread between the Paasche and Laspeyres indexes. As Figure 1,Figure 2Figure 3 highlight, the quadratic-mean-of-order- $r$ index starts showing distinctly different pictures from those indexes when $r$ approaches 10 for the case of output index, 20 for the case of input index and 7 for the case of productivity index. Moreover, Table 2Table 3Table 4 show that the quadratic-mean-of-order- $r$ indexes start fluctuating widely, when $r$ is around 20 or larger. The growth rates of output and input index change even from positive to negative value or vice versa. ${ }^{81}$

We also found that while the spread among the quadratic-mean-of-order- $r$ index is likely to be small for a longer time period, it could become significantly larger even during very short periods of time. The difference in the cumulative growth for the entire period 2002-2017 between the index for $r=2$ and the index for $r=20$ is 3.13 percent for output index, 0.52 percent for input index, and 4.03 percent for productivity index. On the other hand, the difference in the cumulative growth rate during the great recession (2007-2009) between the index for $r=2$ and the index for $r=20$ is 2.42 percent for output index, 1.23 percent for input index, and 4.04 percent for productivity index. Thus, the magnitude of the cumulative difference made for the two years of the great recession is comparable with that accumulated for the 15 years.

Thus for this particular example, all quadratic-mean-of-order- $r$ indexes approximate each other well and are almost the same for the range of $r$ from 0.5 to 5 . On the other

\footnotetext{
${ }^{77}$ More detailed comparisons under a different specification of $r$ is available upon request.

${ }^{78}$ Interestingly, the Törnqvist index, which is another popular superlative index, is shown to be slightly different from those indexes.

${ }^{79}$ The quadratic-mean-of-order-r output index exceeds the spread between the Paasche and Laspyeres indexes in some years even in the range of $r$ from 0.5 to 5 (for example, $r=3$ ). However, the dispersion from those indexes is very small.

${ }^{80}$ It is also worth noting that so far no studies examine the Paasche and Laspeyres bounding test for productivity index.

${ }^{81}$ Since the drastic changes of output and input indexes are somehow cancelled in the productivity index, $r$ needs to be much larger, say 50 (for this data set), so that the sign of the growth rate of productivity changes. See Table 4.
} 
hand, as $r$ gets bigger, the spread between them expands. Indeed, note that especially for some large $r$ (e.g., such as 20 for this data set), the quadratic-mean-of-order- $r$ index could lead to somewhat unreasonable estimates. This is consistent with what Diewert (1978) and Hill (2006) find for the price index. Here we see that this conclusion applies not only to the output and the input indexes but also to the productivity index.

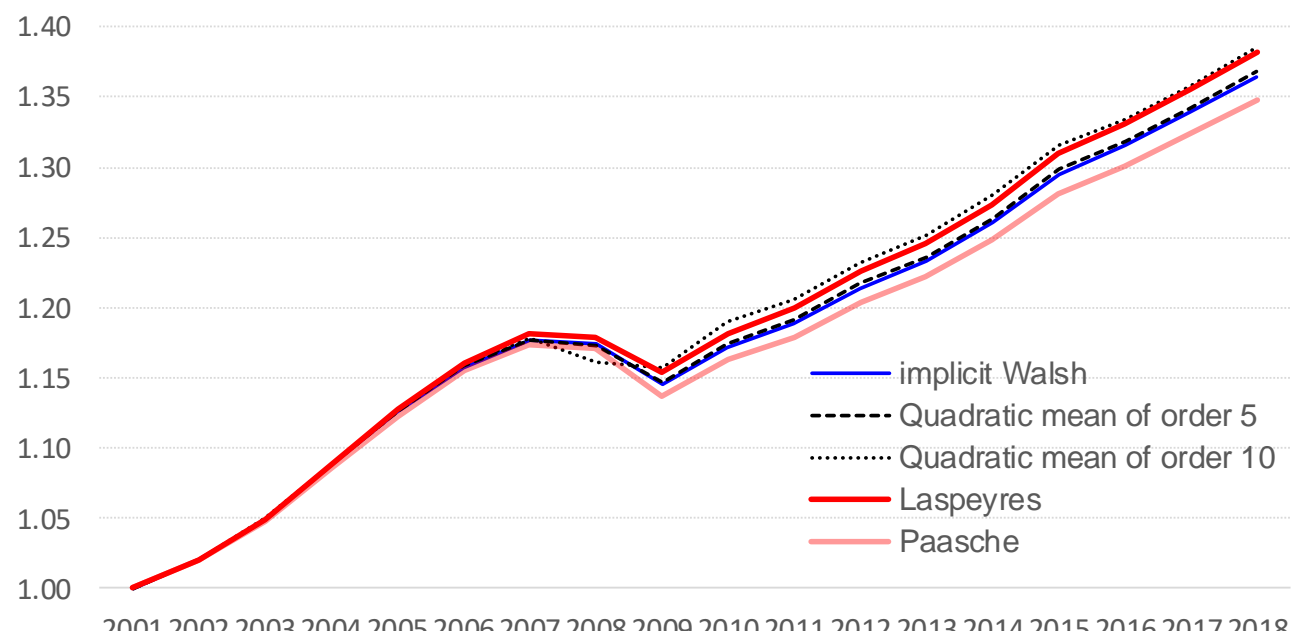

Figure 1: Comparison of output (index 2001=1)

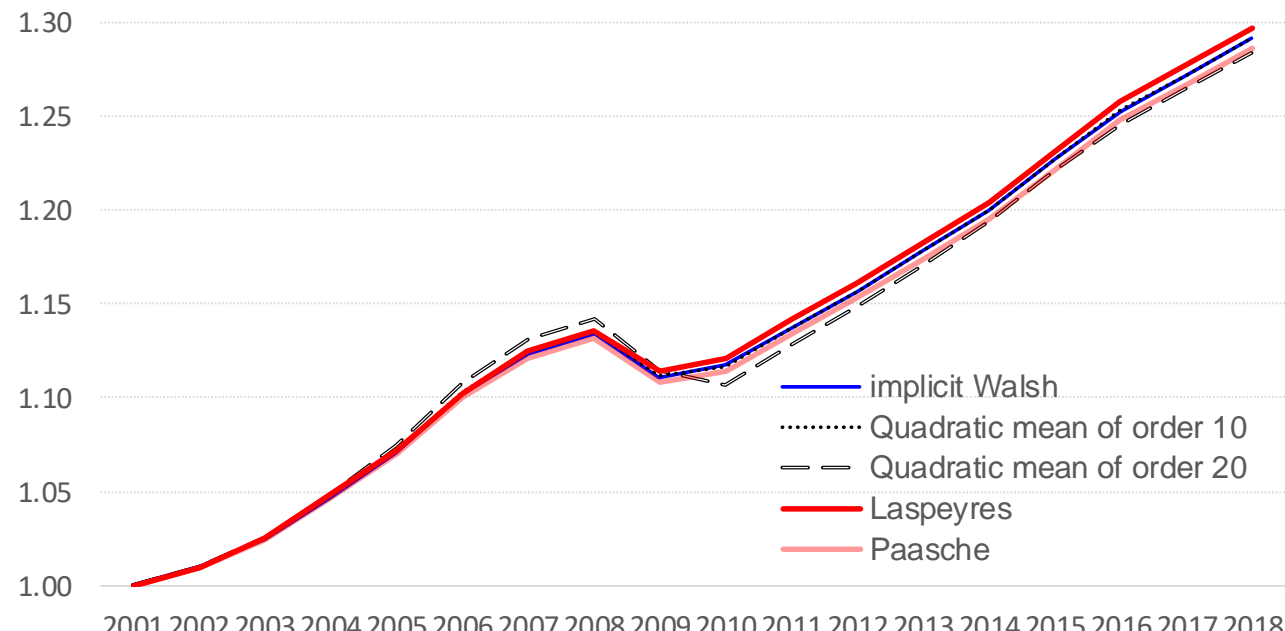

Figure 2: Comparison of input (index 2001=1)

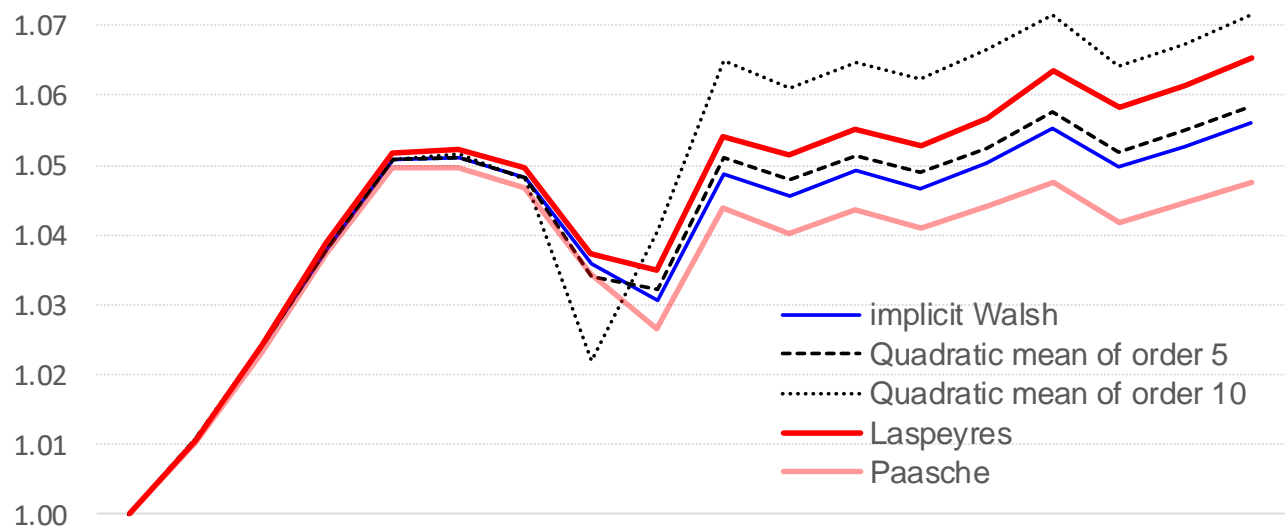

200120022003200420052006200720082009201020112012201320142015201620172018

Figure 3: Comparison of productivity (index 2001=1) 


\section{Concluding Remarks}

The Fisher and Törnqvist indexes of output, input and productivity were known to be only superlative indexes for the corresponding Malmquist indexes. The present paper deals with the quadratic-mean-of-order- $r$ indexes, which are families of empirical indexes of output, input and productivity. We show that all indexes of this family coincide with the corresponding Malmquist indexes under a new family of functional forms based on the quadratic-mean-of-order- $r$, which is shown to be a flexible functional form. Therefore, this generalizes the existing results of superlative indexes, offering many more alternatives to practitioners.

The quadratic-mean-of-order- $r$ index includes many important index numbers as special cases. Most importantly, two indexes deserve a mention here. When $r=2$, that index is reduced to the Fisher index, which Diewert (1992) shows is a superlative index. When $r=1$, that index is reduced to the implicit Walsh index. Although the implicit Walsh index has been recognized before, it has not attracted much attention and its axiomatic properties are not well exploited.

The implicit Walsh quantity index is the change in a value aggregate deflated by the Walsh price index. Thus, measuring quantity changes by the implicit Walsh quantity index implies measuring price changes by the Walsh price index. Therefore, as Diewert (1976) provides a theoretical justification to the Walsh price index by showing that the implicit Walsh quantity index is a superlative index, our result also justifies measuring output and input price changes by using the Walsh price index. ${ }^{82}$

All propositions for superlative indexes need to impose several restrictions on the parameters of underlying distance function. These restrictions specify the point where the distance function can approximate an arbitrary function to the second order. We require either the period 0 distance function to be flexible at the period 0 observation or the period 1 distance function to be flexible at the period 1 observation. These are deemed as reasonable restrictions.

By finding an entire family of superlative indexes, we enrich the applicable instruments for analysing output and input growth and productivity growth. On the other hand, it naturally raises the question of which superlative index one should use? Diewert (1978) shows that all quadratic-mean-of-order- $r$ quantity indexes approximate each other to the second order when price and quantity vectors are fixed over time. He suggests that all these superlative indexes are similar when the changes in prices and quantities are small between the two periods. Meanwhile, Hill (2006) demonstrates that the difference among superlative indexes can be significant and sometimes even bigger than that between the Laspeyres and Paasche indexes by using two data sets from US national accounts and OECD. ${ }^{83}$ Using the most recent US industry-level production accounts, we arrive at similar conclusions for the indexes of output, input and productivity.

\footnotetext{
${ }^{82}$ Statistics Sweden switched to the Walsh price index as its consumer price index only in 2005 (Edvinsson and Södergerg (2011)). As pointed out by Dalën (1999), one of the main reason for this change is that Diewert (1976) justifies using the Walsh price index. Thus, the results of this paper allows one to use the Walsh price index as other price statistics such as the GDP deflator and the Producer Price Index.

${ }^{83}$ Hill (2006) points out that as the parameter $r$ increases in absolute value, the quadratic-mean-of-order$r$ index becomes increasingly sensitive to outliers in the distribution of price and quantity changes. In the range $0 \leq r \leq 2$, these superlative indexes are relatively unaffected by outliers and the difference among them almost never exceeds that between Paasche and Laspeyres indexes.
} 
Finally, an interesting future direction of research would be to combine the economic approach (which we used here to justify the use of superlative indexes with axiomatic approach. Indeed, the existence of a large number of superlative indexes demonstrated by the present paper reminds us about the limitation of solely relying on the economic approach and confirms the importance of combining the economic and axiomatic approaches (and possibly other approaches) for selecting an appropriate empirical index that will measure the reality in the best feasible way.

\section{Acknowledgements}

We thank Knox Lovell, Antonio Peyrache, Prasada Rao, Christopher O'Donnell and seminar participants in 2017 CEPA International Workshop on Performance Analysis: Theory and Practice, November 2017, Brisbane, Australia. Hideyuki Mizobuchi acknowledges the financial support from Japan Society for the Promotion of Science: Grant-in-Aid for Scientific Research (C) (18K01552) and a grant-in-aid from Zengin Foundation for Studies on Economics and Finance. Valentin Zelenyuk acknowledges the financial support from the Australian Research Council (ARC FT170100401) and from The University of Queensland. All remaining errors are the authors' responsibility. 


\section{References}

Allen, R.C. and Diewert, W.E., (1981). "Direct versus Implicit Superlative Index Number Formulae." The Review of Economics and Statistics, Vol.63, No.3, p.430.

Balk, B.M., (1989). "Changing Consumer Preferences and the Cost-of-Living Index: Theory and Nonparametric Expressions." Journal of Economics, Vol.50, No.2, pp.157-169.

Balk, B.M., (1998). Industrial Price, Quantity, and Productivity Indices: The MicroEconomic Theory and an Application, Boston, MA: Springer US.

Balk, B.M., (1993). "Malmquist Productivity Indexes and Fisher Ideal Indexes: Comment." Economic Journal, Vol.103, No.418, p.680.

Balk, B.M., (2008). Price and Quantity Index Numbers, New York, NY: Cambridge University Press.

Barnett, W.A. and Choi, K.H., (2008). "Operational Identification of the Complete Class of Superlative Index Numbers: An Application of Galois Theory." Journal of Mathematical Economics, Vol.44, No.7-8, pp.603-612.

Bjurek, H., (1996). "The Malmquist Total Factor Productivity Index." Scandinavian Journal of Economics, Vol.98, No.2, pp.303-313.

Boskin, M.J. et al., (1996). Toward a More Accurate Measure of the Cost of Living: Final Report, Washington, DC: Government Printing Office for the U.S. Senate Committee on Finance.

Caves, D.W., Christensen, L.R. and Diewert, W.E., (1982). "The Economic Theory of Index Numbers and the Measurement of Input, Output, and Productivity." Econometrica, Vol.50, No.6, pp.1393-1414.

Chambers, R.G., (1988). Applied Production Analysis: A Dual Approach, Cambridge University Press.

Christensen, L.R., Jorgenson, D.W. and Lau, L.J., (1973). "Transcendental Logarithmic Production Frontiers." Review of Economics and Statistics, Vol.55, No.1, pp.2845.

Dalën, J., (1999). "A Proposal for a New System of Aggregation in the Swedish Consumer Price Index." In Paper Presented at the Fifth Meeting of the International Working Group on Price Indexes, Reykjavik, Iceland, August 25-7.

Diewert, W.E., (1980). “Aggregation Problems in the Measurement of Capital.” In D. Usher, ed. The Measurement of Capital. Chicago and London: University of Chicago Press, pp. 433-538.

Diewert, W.E., (1971). "An Application of the Shephard Duality Theorem: A Generalized Leontief Proudction Function." Journal of Political Economy, Vol.79, No.3, pp.481-507.

Diewert, W.E., (1976). "Exact and Superlative Index Numbers." Journal of Econometrics, Vol.4, No.2, pp.115-145.

Diewert, W.E., (1992). "Fisher Ideal Output, Input, and Productivity Indexes Revisited." Journal of Productivity Analysis, Vol.3, No.3, pp.211-248.

Diewert, W.E., (1973). "Functional Forms for Profit and Transformation Functions." Journal of Economic Theory, Vol.6, No.3, pp.284-316. 
Diewert, W.E., (1974). "Functional Forms for Revenue and Factor Requirements Functions.” International Economic Review, Vol.15, No.1, pp.119-130.

Diewert, W.E., (1993a). "Overview of Volume I." In Essays in Index Number Theor Vol. 1. Amsterdam: North-Holland, pp. 1-37.

Diewert, W.E., (1978). "Superlative Index Numbers and Consistency in Aggregation." Econometrica, Vol.46, No.4, pp.883-900.

Diewert, W.E., (1993b). “The Early History of Price Index Research.” In W. E. Diewert and A. O. Nakamura, eds. Essays in Index Number Theor Vol. 1. Amsterdam: North-Holland, pp. 33-71.

Diewert, W.E. and Fox, K.J., (2017). "Decomposing Productivity Indexes into Explanatory Factors." European Journal of Operational Research, Vol.256, No.1, pp.275-291.

Diewert, W.E. and Fox, K.J., (2010). "Malmquist and Törnqvist Productivity Indexes: Returns to Scale and Technical Progress with Imperfect Competition." Journal of Economics, Vol.101, No.1, pp.73-95.

Diewert, W.E. and Fox, K.J., (2014). "Reference Technology Sets, Free Disposal Hulls and Productivity Decompositions." Economics Letters, Vol.122, No.2, pp.238242.

Diewert, W.E. and Morrison, C.J., (1986). “Adjusting Output and Productivity Indexes for Changes in the Terms of Trade.” Economic Journal, Vol.96, No.383, p.659.

Diewert, W.E. and Wales, T.J., (1987). "Flexible Functional Forms and Global Curvature Conditions." Econometrica, Vol.55, No.1, pp.43-68.

Edvinsson, R. and Södergerg, J., (2011). “A Consumer Price Index for Sweden, 12902008." Review of Income and Wealth, Vol.57, No.2, pp.270-292.

Färe, R. et al., (1994). "Productivity Growth, Technical Progress and Efficiency Change in Industrialized Countries." American Economic Review, Vol.84, No.1, pp.66-83.

Färe, R. and Grosskopf, S., (1996). Intertemporal Production Frontiers: With Dynamic $D E A$, Boston, MA: Kluwer Academic Publishers.

Färe, R., Grosskopf, S. and Russell, R.R., (1998). Index Numbers: Essays in Honour of Sten Malmquist, Boston, MA: Kluwer Academic Publishers.

Färe, R. and Primont, D., (1995). Multi-Output Production and Duality: Theory and Applications, Boston, MA: Kluwer Academic Publishers.

Färe, R. and Sung, K.J., (1986). “On Second-Order Taylor's-Series Approximation and Linear Homogeneity.” Aequationes Mathematicae, Vol.30, pp.180-186.

Fisher, I., (1922). The Making of Index Numbers: A Study of Their Varieties, Tests, and Reliability, Boston, MA: Houghton Mifflin.

Frisch, R., (1936). "Annual Survey of General Economic Theory: The Problem of Index Numbers.” Econometrica, Vol.4, No.1, pp.1-38.

Frisch, R., (1930). "Necessary and Sufficient Conditions Regarding the Form of an Index Number which shall Meet Certain of Fisher's Tests.” American Statistical Association Journal, Vol.25, pp.397-406. 
Funke, H., Hacker, G. and Voeller, J., (1979). "Fisher' s Circular Test Reconsidered." Swiss Journal of Economics and Statistics, Vol.115, No.4.

Grifell-Tatjé, E. et al., (2016). "Exact Relationships Between Fisher Indexes and Theoretical Indexes." In J. Aparicio, C. A. K. Lovell, and J. T. Pastor, eds. Advances in Efficiency and Productivity. Cham: Springer, Cham, pp. 97-120.

Hardy, G.H., Littlewood, J.E. and Polya, G., (1934). Inequalities Second., Cambridge, UK: Cambridge University Press.

Hasenkamp, G., (1976). “A Study of Multiple-Output Production Functions.” Journal of Econometrics, Vol.4, No.3, pp.253-262.

Henderson, D.J. and Russell, R.R., (2005). "Human Capital and Convergence: A Production-Frontier Approach." International Economic Review, Vol.46, No.4, pp.1167-1205.

Hill, R.J., (2006). "Superlative Index Numbers: Not All of Them Are Super." Journal of Econometrics, Vol.130, No.1, pp.25-43.

International Labour Office et al., (2004). Consumer Price Index Manual: Theory and Practice, Geneva: International Labour Office.

Kadiyala, K.R., (1972). "Production Functions and Elasticity of Substitution.” Southern Economic Journal, Vol.38, No.3, p.281.

Konüs, A.A., (1924). "The Problem of the True Index of the Cost of Living." The Economic Bulletin of the Institute of Economic Conjuncture (in Russian), Vol.910, No.1, pp.64-71.

Konüs, A.A. and Byushgens, S.S., (1926). "K Probleme Pokupatelnoi Cili Deneg." Voprosi Konyunktri, Vol.2, pp.151-172.

Kumar, S. and Russell, R.R., (2002). "Technological Change, Technological Catch-up, and Capital Deepening: Relative Contributions to Growth and Convergence." American Economic Review, Vol.92, No.3, pp.527-548.

Lau, L.J., (1986). "Functional Forms in Econometric Model Building.” In Z. Griliches and M. D. Intriligator, eds. Handbook of Econometrics. Amsterdam: Elsevier, pp. 1515-1566.

McCarthy, M.D., (1967). "Approximation of the CES Production Function: A Comment." International Economic Review, Vol.8, No.2, p.190.

Mizobuchi, H., (2017). "A Superlative Index Number Formula for the Hicks-Moorsteen Productivity Index." Journal of Productivity Analysis, Vol.48, No.2-3, pp.167178.

Serletis, A. and Feng, G., (2015). "Imposing Theoretical Regularity on Flexible Functional Forms.” Econometric Reviews, Vol.34, pp.198-227.

Serletis, A. and Isakin, M., (2017). "Stochastic Volatility Demand Systems." Econometric Reviews, Vol.36, No.10, pp.1111-1122.

Shephard, R.W., (1953). Cost and Production Functions, Princeton, NJ: Princeton University Press.

Shephard, R.W., (1970). Theory of Cost and Production Function, Princeton, NJ: Princeton University Press. 
Sickles, R.C. and Zelenyuk, V., (2019). Measurement of Productivity and Efficiency, New York: Cambridge University Press. 


\section{Appendix}

\section{Proof of Proposition 1:}

Since $p^{0} \gg 0, p^{1} \gg 0, y^{1}>0$ and $y^{1}>0, \operatorname{IWOI}\left(p^{0}, p^{1}, y^{0}, y^{1}\right)>0$ by definition (35). Thus, $\operatorname{IWOI}\left(p^{0}, p^{1}, y^{0}, y^{1}\right)$ satisfies T1.

By definition (35), IWOI $\left(p^{0}, p^{1}, y^{0}, y^{1}\right)$ is continuous in $p^{0}, p^{1}, y^{1}$ and $y^{1}$. Thus, $\operatorname{IWOI}\left(p^{0}, p^{1}, y^{0}, y^{1}\right)$ satisfies T2.

$\operatorname{IWOI}\left(p^{0}, p^{1}, y, y\right)=\left(\frac{\sum_{m=1}^{M} p_{m}^{1} y_{m}}{\sum_{m=1}^{M} p_{m}^{0} y_{m}}\right) /\left(\frac{\sum_{m=1}^{M}\left(y_{m} y_{m}\right)^{1 / 2} p_{m}^{1}}{\sum_{m=1}^{M}\left(y_{m} y_{m}\right)^{1 / 2} p_{m}^{0}}\right)=$ $\left(\frac{\sum_{m=1}^{M} p_{m}^{1} y_{m}}{\sum_{m=1}^{M} p_{m}^{0} y_{m}}\right) /\left(\frac{\sum_{m=1}^{M} p_{m}^{1} y_{m}}{\sum_{m=1}^{M} p_{m}^{0} y_{m}}\right)=1$. Thus, IWOI $\left(p^{0}, p^{1}, y^{0}, y^{1}\right)$ satisfies T3.

$\operatorname{IWOI}\left(p, p, y^{0}, y^{1}\right)=\left(\frac{\sum_{m=1}^{M} p_{m} y_{m}^{1}}{\sum_{m=1}^{M} p_{m} y_{m}^{0}}\right) /\left(\frac{\sum_{m=1}^{M}\left(y_{m}^{0} y_{m}^{1}\right)^{1 / 2} p_{m}}{\sum_{m=1}^{M}\left(y_{m}^{0} y_{m}^{1}\right)^{1 / 2} p_{m}}\right)=\left(\frac{\sum_{m=1}^{M} p_{m} y_{m}^{1}}{\sum_{m=1}^{M} p_{m} y_{m}^{0}}\right)$

Thus, IWOI $\left(p^{0}, p^{1}, y^{0}, y^{1}\right)$ satisfies T4.

$\operatorname{IWOI}\left(p^{0}, p^{1}, y^{0}, \lambda y^{1}\right)=\left(\frac{\sum_{m=1}^{M} p_{m}^{1} \lambda y_{m}^{1}}{\sum_{m=1}^{M} p_{m}^{0} y_{m}^{0}}\right) /\left(\frac{\sum_{m=1}^{M}\left(y_{m}^{0} \lambda y_{m}^{1}\right)^{1 / 2} p_{m}^{1}}{\sum_{m=1}^{M}\left(y_{m}^{0} \lambda y_{m}^{1}\right)^{1 / 2} p_{m}^{0}}\right)=$ $\left(\frac{\lambda \sum_{m=1}^{M} p_{m}^{1} y_{m}^{1}}{\sum_{m=1}^{M} p_{m}^{0} y_{m}^{0}}\right) /\left(\frac{\lambda^{1 / 2} \sum_{m=1}^{M}\left(y_{m}^{0} y_{m}^{1}\right)^{1 / 2} p_{m}^{1}}{\lambda^{1 / 2} \sum_{m=1}^{M}\left(y_{m}^{0} y_{m}^{1}\right)^{1 / 2} p_{m}^{0}}\right)=\lambda\left(\frac{\sum_{m=1}^{M} p_{m}^{1} y_{m}^{1}}{\sum_{m=1}^{M} p_{m}^{0} y_{m}^{0}}\right) /\left(\frac{\sum_{m=1}^{M}\left(y_{m}^{0} y_{m}^{1}\right)^{1 / 2} p_{m}^{1}}{\sum_{m=1}^{M}\left(y_{m}^{0} y_{m}^{1}\right)^{1 / 2} p_{m}^{0}}\right)=\lambda \times$ $\operatorname{IWOI}\left(p^{0}, p^{1}, y^{0}, y^{1}\right)$. Thus, IWOI $\left(p^{0}, p^{1}, y^{0}, y^{1}\right)$ satisfies T5.

$\operatorname{IWOI}\left(p^{0}, p^{1}, \lambda y^{0}, y^{1}\right)=\left(\frac{\sum_{m=1}^{M} p_{m}^{1} y_{m}^{1}}{\sum_{m=1}^{M} p_{m}^{0} \lambda y_{m}^{0}}\right) /\left(\frac{\sum_{m=1}^{M}\left(\lambda y_{m}^{0} y_{m}^{1}\right)^{1 / 2} p_{m}^{1}}{\sum_{m=1}^{M}\left(\lambda y_{m}^{0} y_{m}^{1}\right)^{1 / 2} p_{m}^{0}}\right)=$ $\left(\frac{\sum_{m=1}^{M} p_{m}^{1} y_{m}^{1}}{\lambda \sum_{m=1}^{M} p_{m}^{0} y_{m}^{0}}\right) /\left(\frac{\lambda^{1 / 2} \sum_{m=1}^{M}\left(y_{m}^{0} y_{m}^{1}\right)^{1 / 2} p_{m}^{1}}{\lambda^{1 / 2} \sum_{m=1}^{M}\left(y_{m}^{0} y_{m}^{1}\right)^{1 / 2} p_{m}^{0}}\right)=\lambda^{-1}\left(\frac{\sum_{m=1}^{M} p_{m}^{1} y_{m}^{1}}{\sum_{m=1}^{M} p_{m}^{0} y_{m}^{0}}\right) /\left(\frac{\sum_{m=1}^{M}\left(y_{m}^{0} y_{m}^{1}\right)^{1 / 2} p_{m}^{1}}{\sum_{m=1}^{M}\left(y_{m}^{0} y_{m}^{1}\right)^{1 / 2} p_{m}^{0}}\right)=$ $\lambda^{-1} I W O I\left(p^{0}, p^{1}, y^{0}, y^{1}\right)$. Thus, IWOI $\left(p^{0}, p^{1}, y^{0}, y^{1}\right)$ satisfies T6.

$\operatorname{IWOI}\left(p^{0}, \lambda p^{1}, y^{0}, y^{1}\right)=\left(\frac{\sum_{m=1}^{M} \lambda p_{m}^{1} y_{m}^{1}}{\sum_{m=1}^{M} p_{m}^{0} y_{m}^{0}}\right) /\left(\frac{\sum_{m=1}^{M}\left(y_{m}^{0} y_{m}^{1}\right)^{1 / 2} \lambda p_{m}^{1}}{\sum_{m=1}^{M}\left(y_{m}^{0} y_{m}^{1}\right)^{1 / 2} p_{m}^{0}}\right)=$ $\left(\lambda \frac{\sum_{m=1}^{M} \lambda p_{m}^{1} y_{m}^{1}}{\sum_{m=1}^{M} p_{m}^{0} y_{m}^{0}}\right) /\left(\lambda \frac{\sum_{m=1}^{M}\left(y_{m}^{0} y_{m}^{1}\right)^{1 / 2} p_{m}^{1}}{\sum_{m=1}^{M}\left(y_{m}^{0} y_{m}^{1}\right)^{1 / 2} p_{m}^{0}}\right)=\left(\frac{\sum_{m=1}^{M} \lambda p_{m}^{1} y_{m}^{1}}{\sum_{m=1}^{M} p_{m}^{0} y_{m}^{0}}\right) /\left(\frac{\sum_{m=1}^{M}\left(y_{m}^{0} y_{m}^{1}\right)^{1 / 2} p_{m}^{1}}{\sum_{m=1}^{M}\left(y_{m}^{0} y_{m}^{1}\right)^{1 / 2} p_{m}^{0}}\right)=$ $\operatorname{IWOI}\left(p^{0}, p^{1}, y^{0}, y^{1}\right)$. Thus, IWOI $\left(p^{0}, p^{1}, y^{0}, y^{1}\right)$ satisfies T7. 
$\operatorname{IWOI}\left(\lambda p^{0}, p^{1}, y^{0}, y^{1}\right)=\left(\frac{\sum_{m=1}^{M} p_{m}^{1} y_{m}^{1}}{\sum_{m=1}^{M} \lambda p_{m}^{0} y_{m}^{0}}\right) /\left(\frac{\sum_{m=1}^{M}\left(y_{m}^{0} y_{m}^{1}\right)^{1 / 2} p_{m}^{1}}{\sum_{m=1}^{M}\left(y_{m}^{0} y_{m}^{1}\right)^{1 / 2} \lambda p_{m}^{0}}\right)=$ $\left(\lambda^{-1} \frac{\sum_{m=1}^{M} p_{m}^{1} y_{m}^{1}}{\sum_{m=1}^{M} p_{m}^{0} y_{m}^{0}}\right) /\left(\lambda^{-1} \frac{\sum_{m=1}^{M}\left(y_{m}^{0} y_{m}^{1}\right)^{1 / 2} p_{m}^{1}}{\sum_{m=1}^{M}\left(y_{m}^{0} y_{m}^{1}\right)^{1 / 2} p_{m}^{0}}\right)=\left(\frac{\sum_{m=1}^{M} p_{m}^{1} y_{m}^{1}}{\sum_{m=1}^{M} p_{m}^{0} y_{m}^{0}}\right) /\left(\frac{\sum_{m=1}^{M}\left(y_{m}^{0} y_{m}^{1}\right)^{1 / 2} p_{m}^{1}}{\sum_{m=1}^{M}\left(y_{m}^{0} y_{m}^{1}\right)^{1 / 2} p_{m}^{0}}\right)=$ $\operatorname{IWOI}\left(p^{0}, p^{1}, y^{0}, y^{1}\right)$. Thus, $\operatorname{IWOI}\left(p^{0}, p^{1}, y^{0}, y^{1}\right)$ satisfies T8.

$\operatorname{IWOI}\left(p^{0}, p^{1}, y^{0}, y^{1}\right)=\left(\frac{\sum_{m=1}^{M} p_{m}^{1} y_{m}^{1}}{\sum_{m=1}^{M} p_{m}^{0} y_{m}^{0}}\right) /\left(\frac{\sum_{m=1}^{M}\left(y_{m}^{0} y_{m}^{1}\right)^{1 / 2} p_{m}^{1}}{\sum_{m=1}^{M}\left(y_{m}^{0} y_{m}^{1}\right)^{1 / 2} p_{m}^{0}}\right)=$

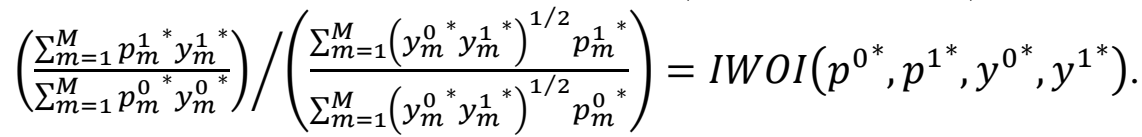

Thus, IWOI $\left(p^{0}, p^{1}, y^{0}, y^{1}\right)$ satisfies T9.

$\operatorname{IWOI}\left(\alpha_{1} p_{1}^{0}, \ldots, \alpha_{M} p_{M}^{0}, \alpha_{1} p_{1}^{1}, \ldots, \alpha_{M} p_{M}^{1}, \alpha_{1}^{-1} y_{1}^{0}, \ldots, \alpha_{M}^{-1} y_{M}^{0}, \alpha_{1}^{-1} y_{1}^{1}, \ldots, \alpha_{M}^{-1} y_{M}^{1}\right)=$ $\left(\frac{\sum_{m=1}^{M} \alpha_{m} \alpha_{m}^{-1} p_{m}^{1} y_{m}^{1}}{\sum_{m=1}^{M} \alpha_{m} \alpha_{m}^{-1} p_{m}^{0} y_{m}^{0}}\right) /\left(\frac{\sum_{m=1}^{M}\left(\alpha_{m}^{-1} y_{m}^{0} \alpha_{m}^{-1} y_{m}^{1}\right)^{1 / 2} \alpha_{m} p_{m}^{1}}{\sum_{m=1}^{M}\left(\alpha_{m}^{-1} y_{m}^{0} \alpha_{m}^{-1} y_{m}^{1}\right)^{1 / 2} \alpha_{m} p_{m}^{0}}\right)=$ $\left(\frac{\sum_{m=1}^{M} \alpha_{m} p_{m}^{1} \alpha_{m}^{-1} y_{m}^{1}}{\sum_{m=1}^{M} \alpha_{m} p_{m}^{0} \alpha_{m}^{-1} y_{m}^{0}}\right) /\left(\frac{\sum_{m=1}^{M} \alpha_{m}^{-1} \alpha_{m}\left(y_{m}^{0} y_{m}^{1}\right)^{1 / 2} p_{m}^{1}}{\sum_{m=1}^{M} \alpha_{m}^{-1} \alpha_{m}\left(y_{m}^{0} y_{m}^{1}\right)^{1 / 2} p_{m}^{0}}\right)=\left(\frac{\sum_{m=1}^{M} p_{m}^{1} y_{m}^{1}}{\sum_{m=1}^{M} p_{m}^{0} y_{m}^{0}}\right) /\left(\frac{\sum_{m=1}^{M}\left(y_{m}^{0} y_{m}^{1}\right)^{1 / 2} p_{m}^{1}}{\sum_{m=1}^{M}\left(y_{m}^{0} y_{m}^{1}\right)^{1 / 2} p_{m}^{0}}\right)=$ $\operatorname{IWOI}\left(p^{0}, p^{1}, y^{0}, y^{1}\right)$. Thus, IWOI $\left(p^{0}, p^{1}, y^{0}, y^{1}\right)$ satisfies T10.

$\operatorname{IWOI}\left(p^{0}, p^{1}, y^{0}, y^{1}\right)=\left(\frac{\sum_{m=1}^{M} p_{m}^{1} y_{m}^{1}}{\sum_{m=1}^{M} p_{m}^{0} y_{m}^{0}}\right) /\left(\frac{\sum_{m=1}^{M}\left(y_{m}^{0} y_{m}^{1}\right)^{1 / 2} p_{m}^{1}}{\sum_{m=1}^{M}\left(y_{m}^{0} y_{m}^{1}\right)^{1 / 2} p_{m}^{0}}\right)=$ $1 /\left(\left(\frac{\sum_{m=1}^{M} p_{m}^{0} y_{m}^{0}}{\sum_{m=1}^{M} p_{m}^{1} y_{m}^{1}}\right) /\left(\frac{\sum_{m=1}^{M}\left(y_{m}^{0} y_{m}^{1}\right)^{1 / 2} p_{m}^{0}}{\sum_{m=1}^{M}\left(y_{m}^{0} y_{m}^{1}\right)^{1 / 2} p_{m}^{1}}\right)\right)=1 / \operatorname{IWOI}\left(p^{1}, p^{0}, y^{1}, y^{0}\right)$.

Thus, $\operatorname{IWOI}\left(p^{0}, p^{1}, y^{0}, y^{1}\right)$ satisfies T11.

Let $p^{0}=(1,1,1), p^{1}=(1.2,3,1.3), y^{0}=(1.2,1,2)$, and $p^{1}=(0.7,1.1,3)$. Then $\operatorname{IWOI}\left(p^{0}, p^{1}, y^{0}, y^{1}\right)=1.1373558$ and $\operatorname{IWOI}\left(p^{1}, p^{0}, y^{1}, y^{0}\right)=1.1475965$. Thus, $\operatorname{IWOI}\left(p^{0}, p^{1}, y^{0}, y^{1}\right) \neq \operatorname{IWOI}\left(p^{1}, p^{0}, y^{0}, y^{1}\right)$. Thus, IWOI $\left(p^{0}, p^{1}, y^{0}, y^{1}\right)$ does not satisfy $\mathrm{T} 12$.

$$
\begin{aligned}
& \left(\frac{\sum_{m=1}^{M} p_{m}^{1} y_{m}^{1}}{\sum_{m=1}^{M} p_{m}^{0} y_{m}^{0}}\right) / I \text { IWOI }\left(p^{0}, p^{1}, y^{0}, y^{1}\right)= \\
& \left(\frac{\sum_{m=1}^{M} p_{m}^{1} y_{m}^{1}}{\sum_{m=1}^{M} p_{m}^{0} y_{m}^{0}}\right) /\left(\left(\frac{\sum_{m=1}^{M} p_{m}^{1} y_{m}^{1}}{\sum_{m=1}^{M} p_{m}^{0} y_{m}^{0}}\right) /\left(\frac{\sum_{m=1}^{M}\left(y_{m}^{0} y_{m}^{1}\right)^{1 / 2} p_{m}^{1}}{\sum_{m=1}^{M}\left(y_{m}^{0} y_{m}^{1}\right)^{1 / 2} p_{m}^{0}}\right)\right)= \\
& \left(\frac{\sum_{m=1}^{M} p_{m}^{1} y_{m}^{1}}{\sum_{m=1}^{M} p_{m}^{0} y_{m}^{0}}\right)\left(\frac{\sum_{m=1}^{M} p_{m}^{0} y_{m}^{0}}{\sum_{m=1}^{M} p_{m}^{1} y_{m}^{1}}\right)\left(\frac{\sum_{m=1}^{M}\left(y_{m}^{0} y_{m}^{1}\right)^{1 / 2} p_{m}^{1}}{\sum_{m=1}^{M}\left(y_{m}^{0} y_{m}^{1}\right)^{1 / 2} p_{m}^{0}}\right)=\left(\frac{\sum_{m=1}^{M}\left(y_{m}^{0} y_{m}^{1}\right)^{1 / 2} p_{m}^{1}}{\sum_{m=1}^{M}\left(y_{m}^{0} y_{m}^{1}\right)^{1 / 2} p_{m}^{0}}\right)= \\
& \left(\frac{\sum_{m=1}^{M} p_{m}^{0} y_{m}^{1}}{\sum_{m=1}^{M} p_{m}^{1} y_{m}^{0}}\right)\left(\frac{\sum_{m=1}^{M} p_{m}^{0} y_{m}^{1}}{\sum_{m=1}^{M} p_{m}^{1} y_{m}^{0}}\right)\left(\frac{\sum_{m=1}^{M}\left(y_{m}^{0} y_{m}^{1}\right)^{1 / 2} p_{m}^{1}}{\sum_{m=1}^{M}\left(y_{m}^{0} y_{m}^{1}\right)^{1 / 2} p_{m}^{0}}\right)=
\end{aligned}
$$




\section{$\left(\frac{\sum_{m=1}^{M} p_{m}^{0} y_{m}^{1}}{\sum_{m=1}^{M} p_{m}^{1} y_{m}^{0}}\right) /\left(\left(\frac{\sum_{m=1}^{M} p_{m}^{1} y_{m}^{0}}{\sum_{m=1}^{M} p_{m}^{0} y_{m}^{1}}\right) /\left(\frac{\sum_{m=1}^{M}\left(y_{m}^{0} y_{m}^{1}\right)^{1 / 2} p_{m}^{1}}{\sum_{m=1}^{M}\left(y_{m}^{0} y_{m}^{1}\right)^{1 / 2} p_{m}^{0}}\right)\right)=$ $\left(\frac{\sum_{m=1}^{M} p_{m}^{0} y_{m}^{1}}{\sum_{m=1}^{M} p_{m}^{1} y_{m}^{0}}\right) / I W O I\left(p^{0}, p^{1}, y^{1}, y^{0}\right)$.}

Thus, IWOI $\left(p^{0}, p^{1}, y^{0}, y^{1}\right)$ satisfies T13.

$$
\begin{aligned}
& I W O I\left(p^{0}, p^{1}, y^{0}, y^{1}\right)=\left(\frac{\sum_{m=1}^{M} p_{m}^{1} y_{m}^{1}}{\sum_{m=1}^{M} p_{m}^{0} y_{m}^{0}}\right)\left(\frac{\sum_{m=1}^{M}\left(y_{m}^{0} y_{m}^{1}\right)^{1 / 2} p_{m}^{0}}{\sum_{m=1}^{M}\left(y_{m}^{0} y_{m}^{1}\right)^{1 / 2} p_{m}^{1}}\right)= \\
& \left(\left(\sum_{m=1}^{M} \frac{p_{m}^{0} y_{m}^{0}}{\sum_{j=1}^{M} p_{j}^{0} y_{j}^{0}}\left(\frac{y_{m}^{1}}{y_{m}^{0}}\right)^{1 / 2}\right)^{2}\left(\sum_{m=1}^{M} \frac{p_{m}^{1} y_{m}^{1}}{\sum_{j=1}^{M} p_{j}^{1} y_{j}^{1}}\left(\frac{y_{m}^{1}}{y_{m}^{0}}\right)^{-1 / 2}\right)^{-2}\right)^{1 / 2} .
\end{aligned}
$$

Thus, IWOI $\left(p^{0}, p^{1}, y^{0}, y^{1}\right)$ can be considered as the geometric mean of the weighted mean of order 2 and the weighted mean of order -2 . As Hardy et al. (1934) verify, both of them are ranged from the minimum output relative to the maximum output relative so that $\min \left(\frac{y_{1}^{1}}{y_{1}^{0}}, \ldots, \frac{y_{M}^{1}}{y_{M}^{0}}\right) \leq\left(\sum_{m=1}^{M} \frac{p_{m}^{0} y_{m}^{0}}{\sum_{j=1}^{M} p_{j}^{0} y_{j}^{0}}\left(\frac{y_{m}^{1}}{y_{m}^{0}}\right)^{1 / 2}\right)^{2} \leq \max \left(\frac{y_{1}^{1}}{y_{1}^{0}}, \ldots, \frac{y_{M}^{1}}{y_{M}^{0}}\right) \quad$ and $\min \left(\frac{y_{1}^{1}}{y_{1}^{0}}, \ldots, \frac{y_{M}^{1}}{y_{M}^{0}}\right) \leq\left(\sum_{m=1}^{M} \frac{p_{m}^{1} y_{m}^{1}}{\sum_{j=1}^{M} p_{j}^{1} y_{j}^{1}}\left(\frac{y_{m}^{1}}{y_{m}^{0}}\right)^{-1 / 2}\right)^{-2} \leq \max \left(\frac{y_{1}^{1}}{y_{1}^{0}}, \ldots, \frac{y_{M}^{1}}{y_{M}^{0}}\right)$.

Therefore, IWOI $\left(p^{0}, p^{1}, y^{0}, y^{1}\right)$ satisfies T14.

The second term of the inequality of T15 indicates the Walsh output price index so that $\frac{\left(\sum_{m=1}^{M} p_{m}^{1} y_{m}^{1} / \sum_{m=1}^{M} p_{m}^{0} y_{m}^{0}\right)}{I W O I\left(p^{0}, p^{1}, y^{0}, y^{1}\right)}=\left(\frac{\sum_{m=1}^{M}\left(y_{m}^{0} y_{m}^{1}\right)^{1 / 2} p_{m}^{1}}{\sum_{m=1}^{M}\left(y_{m}^{0} y_{m}^{1}\right)^{1 / 2} p_{m}^{0}}\right)$. Let $\frac{p_{\min }^{1}}{p_{\min }^{0}}=\min \left(\frac{p_{1}^{1}}{p_{1}^{0}}, \ldots, \frac{p_{M}^{1}}{p_{M}^{0}}\right)$ and $\frac{p_{\max }^{1}}{p_{\max }^{0}}=\max \left(\frac{p_{1}^{1}}{p_{1}^{0}}, \ldots, \frac{p_{M}^{1}}{p_{M}^{0}}\right)$. By definition, we can derive $\left(\frac{\sum_{m=1}^{M}\left(y_{m}^{0} y_{m}^{1}\right)^{1 / 2} p_{m}^{1}}{\sum_{m=1}^{M}\left(y_{m}^{0} y_{m}^{1}\right)^{1 / 2} p_{m}^{0}}\right) \geq$ $\left(\frac{\sum_{m=1}^{M}\left(y_{m}^{0} y_{m}^{1}\right)^{1 / 2} p_{\min }^{1}}{\sum_{m=1}^{M}\left(y_{m}^{0} y_{m}^{1}\right)^{1 / 2} p_{\min }^{0}}\right)=\frac{p_{\min }^{1}}{p_{\min }^{0}}$ and $\left(\frac{\sum_{m=1}^{M}\left(y_{m}^{0} y_{m}^{1}\right)^{1 / 2} p_{m}^{1}}{\sum_{m=1}^{M}\left(y_{m}^{0} y_{m}^{1}\right)^{1 / 2} p_{m}^{0}}\right) \leq\left(\frac{\sum_{m=1}^{M}\left(y_{m}^{0} y_{m}^{1}\right)^{1 / 2} p_{\max }^{1}}{\sum_{m=1}^{M}\left(y_{m}^{0} y_{m}^{1}\right)^{1 / 2} p_{\max }^{0}}\right)=\frac{p_{\max }^{1}}{p_{\max }^{0}}$. It implies the inequality of T15. Thus, $\operatorname{IWOI}\left(p^{0}, p^{1}, y^{0}, y^{1}\right)$ satisfies T15.

Let $p^{0}=(1,1,1), p^{1}=(1.2,3,1.3), y^{0}=(1.2,1,2)$, and $p^{1}=(0.7,1.1,3)$. Then $\frac{\sum_{m=1}^{M} p_{m}^{0} y_{m}^{1}}{\sum_{m=1}^{M} p_{m}^{0} y_{m}^{0}}=1.1428571, \quad \frac{\sum_{m=1}^{M} p_{m}^{1} y_{m}^{1}}{\sum_{m=1}^{M} p_{m}^{1} y_{m}^{0}}=1.1420455$ and $\operatorname{IWOI}\left(p^{0}, p^{1}, y^{0}, y^{1}\right)=$ 1.1373558 . Thus, IWOI $\left(p^{0}, p^{1}, y^{0}, y^{1}\right) \leq \frac{\sum_{m=1}^{M} p_{m}^{1} y_{m}^{1}}{\sum_{m=1}^{M} p_{m}^{1} y_{m}^{0}} \leq \frac{\sum_{m=1}^{M} p_{m}^{0} y_{m}^{1}}{\sum_{m=1}^{M} p_{m}^{0} y_{m}^{0}} \quad$. Thus, $\operatorname{IWOI}\left(p^{0}, p^{1}, y^{0}, y^{1}\right)$ does not satisfy T16.

Let $p^{0}=(1,1,1), p^{1}=(0.8,1.1,3), y^{0}=(1.2,1,2), y^{1}=(0.01,12,0.00004)$ and $y^{2}=(0.101,12.000001,0.000041)$. Then $\operatorname{IWOI}\left(p^{0}, p^{1}, y^{0}, y^{1}\right)=2.8704594$ and $\operatorname{IWOI}\left(p^{0}, p^{1}, y^{0}, y^{2}\right)=2.870441 \quad$. Thus, $\quad \operatorname{IWOI}\left(p^{0}, p^{1}, y^{0}, y^{1}\right) \geq$ $\operatorname{IWOI}\left(p^{0}, p^{1}, y^{0}, y^{2}\right)$. Thus, IWOI $\left(p^{0}, p^{1}, y^{0}, y^{1}\right)$ does not satisfy T17.

Let $p^{0}=(1,1,1), p^{1}=(0.1,0.5,10), y^{0}=(0.5,10,2), y^{1}=(0.6,0.002,0.0005)$ and $y^{2}=(0.5001,10.0001,2.0001)$. Then $\operatorname{IWOI}\left(p^{0}, p^{1}, y^{0}, y^{1}\right)=0.00861570$ and 
$\operatorname{IWOI}\left(p^{0}, p^{1}, y^{2}, y^{1}\right)=0.00861590 \quad$. Thus, $\quad \operatorname{IWOI}\left(p^{0}, p^{1}, y^{0}, y^{1}\right) \leq$ $\operatorname{IWOI}\left(p^{0}, p^{1}, y^{2}, y^{1}\right)$. Thus, IWOI $\left(p^{0}, p^{1}, y^{0}, y^{1}\right)$ does not satisfy T18.

Since $\left(\frac{\sum_{m=1}^{M} p_{m}^{t} y_{m}^{1}}{\sum_{m=1}^{M} p_{m}^{s} y_{m}^{0}}\right) / I W O I\left(p^{s}, p^{t}, y^{0}, y^{1}\right)=\frac{\sum_{m=1}^{M}\left(y_{m}^{0} y_{m}^{1}\right)^{1 / 2} p_{m}^{t}}{\sum_{m=1}^{M}\left(y_{m}^{0} y_{m}^{1}\right)^{1 / 2} p_{m}^{s}}$ is the Walsh output price index, we denote it by $\operatorname{WOPI}\left(p^{t}, p^{s}, y^{0}, y^{1}\right)$ in the proof of T19 and T20 below. $\frac{\partial W O P I}{\partial p_{m}^{t}}=\frac{\left(y_{m}^{0} y_{m}^{1}\right)^{1 / 2}}{\sum_{j=1}^{M}\left(y_{j}^{0} y_{j}^{1}\right)^{1 / 2} p_{j}^{s}}>0$ for $m=1, \ldots, M$. Thus, IWOI $\left(p^{0}, p^{1}, y^{0}, y^{1}\right)$ satisfies T19.

Adopting the notation of Proof of T19, $\frac{\partial W O P I}{\partial p_{m}^{s}}=\frac{\sum_{j=1}^{M}\left(y_{j}^{0} y_{j}^{1}\right)^{1 / 2} p_{j}^{t}}{\left(\sum_{j=1}^{M}\left(y_{j}^{0} y_{j}^{1}\right)^{1 / 2} p_{j}^{s}\right)^{2}} \times\left(y_{m}^{0} y_{m}^{1}\right)^{1 / 2}>0$ for $m=1, \ldots, M$. Thus, IWOI $\left(p^{0}, p^{1}, y^{0}, y^{1}\right)$ satisfies T20. QED. 
Proof of Proposition 2: This proposition claims that even under the restrictions of (55)(59), $g_{r}$ can approximate any arbitrary output distance function $D_{o}^{*}$ to the second order at $\left(x^{*}, y^{*}\right)$. Stated differently, the following equations are satisfied: ${ }^{84}$

$$
\begin{gathered}
g_{r}\left(x^{*}, y^{*}\right)=D_{o}^{*}\left(x^{*}, y^{*}\right)=1, \\
\frac{\partial g_{r}\left(x^{*}, y^{*}\right)}{\partial x_{n}}=\frac{\partial D_{o}^{*}\left(x^{*}, y^{*}\right)}{\partial x_{n}}, \forall n \in[1, \ldots, N], \\
\frac{\partial g_{r}\left(x^{*}, y^{*}\right)}{\partial y_{m}}=\frac{\partial D_{o}^{*}\left(x^{*}, y^{*}\right)}{\partial y_{m}}, m \in[1, \ldots, M], \\
\frac{\partial^{2} g_{r}\left(x^{*}, y^{*}\right)}{\partial x_{u} \partial x_{v}}=\frac{\partial^{2} D_{o}^{*}\left(x^{*}, y^{*}\right)}{\partial x_{u} \partial x_{v}}, \forall u, v \in[1, \ldots, N], \\
\frac{\partial^{2} g_{r}\left(x^{*}, y^{*}\right)}{\partial y_{j} \partial y_{k}}=\frac{\partial^{2} D_{o}^{*}\left(x^{*}, y^{*}\right)}{\partial y_{j} \partial y_{k}}, \forall j, k \in[1, \ldots, M], \\
\frac{\partial^{2} g_{r}\left(x^{*}, y^{*}\right)}{\partial x_{n} \partial y_{m}}=\frac{\partial^{2} D_{o}^{*}\left(x^{*}, y^{*}\right)}{\partial x_{n} \partial y_{m}}, \forall n \in[1, \ldots, N], \forall m \in[1, \ldots, M] .
\end{gathered}
$$

We define the parameters in $g_{r}$ as follows: ${ }^{85}$

$$
\begin{gathered}
a_{j, k}=y_{j}^{* 1-r / 2} y_{k}^{* 1-r / 2}\left[\frac{\partial^{2} D_{o}^{*}\left(x^{*}, y^{*}\right)}{\partial y_{j} \partial y_{k}}-(1-r) \frac{\partial D_{o}^{*}\left(x^{*}, y^{*}\right)}{\partial y_{j}} \frac{\partial D_{o}^{*}\left(x^{*}, y^{*}\right)}{\partial y_{k}}\right], \\
\forall j \neq k \in[1, \ldots, M], \\
a_{m, m}=y_{m}^{* 2-r}\left[\frac{\partial^{2} D_{o}^{*}\left(x^{*}, y^{*}\right)}{\partial y_{m}^{2}}-(1-r)\left(\frac{\partial D_{o}^{*}\left(x^{*}, y^{*}\right)}{\partial y_{m}}\right)^{2}+\left(1-\frac{r}{2}\right) \frac{\partial D_{o}^{*}\left(x^{*}, y^{*}\right)}{\partial y_{m}} \frac{1}{y_{m}^{*}}\right], \\
\forall m \in[1, \ldots, M], \\
c_{u, v}=x_{u}^{* 1-r / 2} x_{v}^{* 1-r / 2}\left[-\frac{\partial^{2} D_{o}^{*}\left(x^{*}, y^{*}\right)}{\partial x_{u} \partial x_{v}}+(1+r) \frac{\partial D_{o}^{*}\left(x^{*}, y^{*}\right)}{\partial x_{u}} \frac{\partial D_{o}^{*}\left(x^{*}, y^{*}\right)}{\partial x_{v}}\right], \\
\forall u \neq v \in[1, \ldots, N], \\
c_{n, n}=x_{n}^{* 2-r}\left[-\frac{\partial^{2} D_{o}^{*}\left(x^{*}, y^{*}\right)}{\partial x_{n}^{2}}+(1+r)\left(\frac{\partial D_{o}^{*}\left(x^{*}, y^{*}\right)}{\partial x_{n}}\right)^{2}-(1-\right. \\
\left.\left.\frac{r}{2}\right) \frac{\partial D_{o}^{*}\left(x^{*}, y^{*}\right)}{\partial x_{n}} \frac{1}{x_{n}^{*}}\right], \quad \begin{array}{c}
\forall n \in[1, \ldots, N], \\
\left.b_{m, n}=\frac{4}{r} \frac{y_{m}^{2} D_{o}^{*}\left(x^{*}, y^{*}\right)}{\partial \sum_{m}^{M} \partial x_{n}}+\frac{\partial D_{o}^{*}\left(x^{*}, y^{*}\right)}{\partial y_{m}} \frac{\partial D_{o}^{*}\left(x^{*}, y^{*}\right)}{\partial x_{n}}\right], \\
\forall n \in[1, \ldots, N], \forall m \in[1, \ldots, M] .
\end{array}
\end{gathered}
$$

First, we show that these parameters specified by (112)-(116) satisfy the restrictions (56)- (59). By summing $a_{j, k} y_{k}^{* r / 2}$ over $k$, we can derive the following equation.

$$
\begin{aligned}
& \sum_{k=1}^{M} a_{j, k} y_{k}^{* r / 2}=\sum_{k \neq j} a_{j, k} y_{k}^{* r / 2}+a_{j, j} y_{j}^{* r / 2} \\
& =\sum_{k \neq j} y_{j}^{* 1-r / 2} y_{k}^{*}\left[\frac{\partial^{2} D_{o}^{*}\left(x^{*}, y^{*}\right)}{\partial y_{j} \partial y_{k}}-(1-r) \frac{\partial D_{o}^{*}\left(x^{*}, y^{*}\right)}{\partial y_{j}} \frac{\partial D_{o}^{*}\left(x^{*}, y^{*}\right)}{\partial y_{k}}\right]+ \\
& y_{j}^{* 2-r / 2}\left[\frac{\partial^{2} D_{o}^{*}\left(x^{*}, y^{*}\right)}{\partial y_{j}^{2}}-(1-r)\left(\frac{\partial D_{o}^{*}\left(x^{*}, y^{*}\right)}{\partial y_{j}}\right)^{2}+\left(1-\frac{r}{2}\right) \frac{\partial D_{o}^{*}\left(x^{*}, y^{*}\right)}{\partial y_{j}} \frac{1}{y_{j}^{*}}\right] \\
& \text { from }(112) \text { and (113), } \\
& \begin{array}{r}
\left(1-\frac{r}{2}\right) y_{j}^{* 1-r / 2} \frac{\partial D_{o}^{*}\left(x^{*}, y^{*}\right)}{\partial y_{j}}=\left(-(1-r)+\left(1-\frac{r}{2}\right)\right) y_{j}^{* 1-r / 2} \frac{\partial D_{o}^{*}\left(x^{*}, y^{*}\right)}{\partial y_{j}} \\
\text { from (7), (13) and (14), }
\end{array}
\end{aligned}
$$

\footnotetext{
${ }^{84}$ For simplicity, we adopt the notation such as $\frac{\partial g_{r}\left(x^{*}, y^{*}\right)}{\partial x_{n}}=\left.\frac{\partial g_{r}(x, y)}{\partial x_{n}}\right|_{x=x^{*}, y=y^{*}}$ throughout this paper.

85 This specification of parameters is just an example. There might exist an alternative specification which guarantees the second order approximation property.
} 


$$
=\left(\frac{r}{2}\right) y_{j}^{* 1-r / 2} \frac{\partial D_{o}^{*}\left(x^{*}, y^{*}\right)}{\partial y_{j}} .
$$

Then, it implies (56) as follows:

$$
\begin{aligned}
& \sum_{j=1}^{M} \sum_{k=1}^{M} a_{j, k} y_{j}^{* r / 2} y_{k}^{* r / 2}=\sum_{j=1}^{M} y_{j}^{* r / 2}\left(\frac{r}{2}\right) y_{j}^{* 1-r / 2} \frac{\partial D_{o}^{*}\left(x^{*}, y^{*}\right)}{\partial y_{j}} \\
& =\sum_{j=1}^{M}\left(\frac{r}{2}\right) y_{j}^{*} \frac{\partial D_{o}^{*}\left(x^{*}, y^{*}\right)}{\partial y_{j}}=\frac{r}{2} D_{o}^{*}\left(x^{*}, y^{*}\right)=\frac{r}{2}
\end{aligned}
$$

from (7) and (13).

By summing $c_{u, v} x_{v}^{* r / 2}$ over $v$, we can derive the following equation.

$$
\begin{aligned}
& \sum_{v=1}^{N} c_{u, v} x_{v}^{* r / 2}=\sum_{v \neq u} c_{u, v} x_{v}^{* r / 2}+c_{u, u} x_{u}^{* r / 2} \\
& =\sum_{v \neq u} x_{u}^{* 1-r / 2} x_{v}^{*}\left[-\frac{\partial^{2} D_{o}^{*}\left(x^{*}, y^{*}\right)}{\partial x_{u} \partial x_{v}}+(1+r) \frac{\partial D_{o}^{*}\left(x^{*}, y^{*}\right)}{\partial x_{u}} \frac{\partial D_{o}^{*}\left(x^{*}, y^{*}\right)}{\partial x_{v}}\right]+ \\
& x_{u}^{* 2-r / 2}\left[-\frac{\partial^{2} D_{o}^{*}\left(x^{*}, y^{*}\right)}{\partial x_{u}^{2}}+(1+r)\left(\frac{\partial D_{o}^{*}\left(x^{*}, y^{*}\right)}{\partial x_{u}}\right)^{2}-\left(1-\frac{r}{2}\right) \frac{\partial D_{o}^{*}\left(x^{*}, y^{*}\right)}{\partial x_{u}} \frac{1}{x_{u}^{*}}\right] \\
& \text { from }(114) \text { and }(115), \\
& =x_{u}^{* 1-r / 2} \sum_{v=1}^{N}\left[-\frac{\partial^{2} D_{o}^{*}\left(x^{*}, y^{*}\right)}{\partial x_{u} \partial x_{v}} x_{v}^{*}+(1+r) \frac{\partial D_{o}^{*}\left(x^{*}, y^{*}\right)}{\partial x_{u}} \frac{\partial D_{o}^{*}\left(x^{*}, y^{*}\right)}{\partial x_{v}} x_{v}^{*}\right]- \\
& \left(1-\frac{r}{2}\right) x_{u}^{* 1-r / 2} \frac{\partial D_{o}^{*}\left(x^{*}, y^{*}\right)}{\partial x_{u}} \\
& =\left(-(-2)-(1+r)-\left(1-\frac{r}{2}\right)\right) x_{u}^{* 1-r / 2} \frac{\partial D_{o}^{*}\left(x^{*}, y^{*}\right)}{\partial x_{u}} \\
& \text { from (7), (10) and (11), } \\
& =-\left(\frac{r}{2}\right) x_{u}^{* 1-r / 2} \frac{\partial D_{o}^{*}\left(x^{*}, y^{*}\right)}{\partial x_{u}} .
\end{aligned}
$$

Then, it implies (57) as follows:

$$
\begin{aligned}
& \sum_{u=1}^{N} \sum_{v=1}^{N} c_{u, v} x_{u}^{* r / 2} x_{v}^{* r / 2}=-\sum_{u=1}^{N} x_{u}^{* r / 2}\left(\frac{r}{2}\right) x_{u}^{* 1-r / 2} \frac{\partial D_{o}^{*}\left(x^{*}, y^{*}\right)}{\partial x_{u}} \\
& =-\sum_{u=1}^{N}\left(\frac{r}{2}\right) x_{u}^{*} \frac{\partial D_{o}^{*}\left(x^{*}, y^{*}\right)}{\partial x_{u}}=-\frac{r}{2} \times\left(-D_{o}^{*}\left(x^{*}, y^{*}\right)\right)=\frac{r}{2} D_{o}^{*}\left(x^{*}, y^{*}\right)=\frac{r}{2}
\end{aligned}
$$

from (7) and (10).

By summing $b_{m, n} y_{m}^{* r / 2}$ over $m$, we obtain (58) so as

$$
\begin{aligned}
& \sum_{m=1}^{M} b_{m, n} y_{m}^{* r / 2}=\frac{4}{r} \frac{x_{n}^{* 1-r / 2}}{\left(\sum_{j=1}^{M} \alpha_{j} y_{j}^{* r / 2}\right)\left(\sum_{u=1}^{N} \beta_{u} x_{u}^{*-r / 2}\right)} \times \\
& \sum_{m=1}^{M}\left[-\frac{\partial^{2} D_{o}^{*}\left(x^{*}, y^{*}\right)}{\partial y_{m} \partial x_{n}} y_{m}^{*}+\frac{\partial D_{o}^{*}\left(x^{*}, y^{*}\right)}{\partial y_{m}} y_{m}^{*} \frac{\partial D_{o}^{*}\left(x^{*}, y^{*}\right)}{\partial x_{n}}\right] \\
& =\frac{4}{r} \frac{x_{n}^{* 1-r / 2}}{\left(\sum_{j=1}^{M} \alpha_{j} y_{j}^{* r / 2}\right)\left(\sum_{u=1}^{N} \beta_{u} x_{u}^{*-r / 2}\right)} \times(-1+1) \frac{\partial D_{o}^{*}\left(x^{*}, y^{*}\right)}{\partial x_{n}}=0 \\
& \text { from (116), }
\end{aligned}
$$

By summing $b_{m, n} x_{n}^{*-r / 2}$ over $n$, we obtain (59) so as

$$
\begin{array}{ll}
\sum_{n=1}^{N} b_{m, n} x_{n}^{*-r / 2}=\frac{4}{r} \frac{y_{m}^{*} 1-r / 2}{\left(\sum_{j=1}^{M} \alpha_{j} y_{j}^{* r / 2}\right)\left(\sum_{u=1}^{N} \beta_{u} x_{u}^{*-r / 2}\right)} \times & \\
\sum_{n=1}^{N}\left[-\frac{\partial^{2} D_{o}^{*}\left(x^{*}, y^{*}\right)}{\partial y_{m} \partial x_{n}} x_{n}^{*}+\frac{\partial D_{o}^{*}\left(x^{*}, y^{*}\right)}{\partial y_{m}} \frac{\partial D_{o}^{*}\left(x^{*}, y^{*}\right)}{\partial x_{n}} x_{n}^{*}\right] & \text { from (116), } \\
=\frac{4}{r} \frac{x_{n}^{* 1-r / 2}}{\left(\sum_{j=1}^{M} \alpha_{j} y_{j}^{* r / 2}\right)\left(\sum_{u=1}^{N} \beta_{u} x_{u}^{*-r / 2}\right)} \times(-(-1)-1) \frac{\partial D_{o}^{*}\left(x^{*}, y^{*}\right)}{\partial y_{m}}=0 & \text { from (7), (10) and (12). }
\end{array}
$$


Second, we show that these parameters specified by (112)-(116) satisfy the equations for the second order approximation (106)-(111).

As we show, (112)-(116) imply (56)-(59). Substituting (56)-(59) into (52), we obtain $g_{r}\left(x^{*}, y^{*}\right)=[(r / 2) /(r / 2)]^{1 / r}=1$, which implies (106) along with (7).

The second-order derivatives of $g_{r}$ with respect to inputs $x$ evaluated at $\left(x^{*}, y^{*}\right)$ are given by the following equations, using (56)-(59). ${ }^{86}$

$$
\begin{aligned}
& \frac{\partial^{2} g_{r}\left(x^{*}, y^{*}\right)}{\partial x_{u} \partial x_{v}}=\left((1-r)\left(\frac{r}{2}\right)^{-2}+4\left(\frac{r}{2}\right)^{-1}\right) \times \\
& x_{u}^{* r / 2-1} x_{v}^{* r / 2-1}\left(\sum_{n=1}^{N} c_{u, n} x_{n}^{* r / 2}\right)\left(\sum_{n=1}^{N} c_{v, n} x_{n}^{* r / 2}\right)-c_{u, v} x_{u}^{* r / 2-1} x_{v}^{* r / 2-1} \text {, } \\
& \forall u \neq v \in[1, \ldots, N], \\
& \frac{\partial^{2} g_{r}\left(x^{*}, y^{*}\right)}{\partial x_{n}^{2}}=\left((1-r)\left(\frac{r}{2}\right)^{-2}+4\left(\frac{r}{2}\right)^{-1}\right) \times x_{n}^{* r-2}\left(\sum_{u=1}^{N} c_{n, u} x_{u}^{* r / 2}\right)^{2}+ \\
& -c_{n, n} x_{n}^{* r-2}\left(1-\frac{r}{2}\right)\left(\frac{r}{2}\right)^{-1} x_{n}^{* r / 2-2}\left(\sum_{u=1}^{N} c_{n, u} x_{u}^{* r / 2}\right) \text {, }
\end{aligned}
$$

$\forall n \in[1, \ldots, N]$

Substituting (118) into (119) and (120), we obtain (109).

The second-order derivatives of $g_{r}$ with respect to outputs $y$ evaluated at $\left(x^{*}, y^{*}\right)$ are given by the following equations, using (56)-(59).

$$
\begin{aligned}
& \frac{\partial^{2} g_{r}\left(x^{*}, y^{*}\right)}{\partial y_{j} \partial y_{k}}=(1- \\
& r)\left(\frac{1}{r}\right)^{-2} y_{j}^{* r / 2-1} y_{k}^{* r / 2-1}\left(\sum_{m=1}^{M} a_{j, m} y_{m}^{* r / 2}\right)\left(\sum_{m=1}^{M} a_{k, m} y_{m}^{* r / 2}\right)+ \\
& a_{j, k} y_{j}^{* r / 2-1} y_{k}^{* r / 2-1}, \\
& \frac{\partial^{2} g_{r}\left(x^{*}, y^{*}\right)}{\partial y_{m}^{2}}=(1-r)\left(\frac{1}{r}\right)^{-2} y_{m}^{* r-2}\left(\sum_{j=1}^{M} a_{m, j} y_{j}^{* r / 2}\right)^{2}+a_{m, m} y_{m}^{* r-2}+ \\
& \left(\frac{r}{2}-1\right)\left(\frac{r}{2}\right)^{-1} y_{m}^{* r / 2-2}\left(\sum_{j=1}^{M} a_{m, j} y_{j}^{* r / 2}\right),
\end{aligned}
$$

$\forall m \in[1, \ldots, M]$

Substituting (117) into (121) and (122), we obtain (110).

The second-order cross-derivatives of $g_{r}$ with respect to inputs $x$ and outputs $y$ evaluated at $\left(x^{*}, y^{*}\right)$ are given by the following equation, using (58) and (59).

$$
\begin{aligned}
& \frac{\partial^{2} g_{r}\left(x^{*}, y^{*}\right)}{\partial y_{m} \partial x_{n}}=\left(-2\left(\frac{1}{r}-1\right)\left(\frac{r}{2}\right)^{-1}-2\left(\frac{r}{2}\right)^{-1}\right) \times \\
& y_{m}^{* r / 2-1} x_{n}^{* r / 2-1}\left(\sum_{j=1}^{M} a_{m, j} y_{j}^{* r / 2}\right)\left(\sum_{u=1}^{N} c_{n, u} x_{u}^{* r / 2}\right)- \\
& \left(\frac{1}{2}\right)\left(\sum_{j=1}^{M} \alpha_{j} y_{j}^{* \frac{r}{2}}\right)\left(\sum_{u=1}^{N} \beta_{u} x_{u}^{*-\frac{r}{2}}\right) b_{m, n} y_{m}^{* r / 2-1} x_{n}^{*-r / 2-1}, \\
& \forall m \in[1, \ldots, M], \forall n \in[1, \ldots, N] .
\end{aligned}
$$

Substituting (116)-(118) into (123), we obtain (111).

Since $D_{o}^{*}$ and $g_{r}$ are linearly homogeneous in outputs $y$ and homogeneous of degree minus one in inputs $x,(10)-(15)$ hold for both of them. Equations (11), (12) and (15)

\footnotetext{
${ }^{86}$ Without any restrictions on the parameters on $g_{r}$, its second derivatives are too complicated to be written down explicitly here. The restrictions by (56)-(59) cause their representation to be in much simpler forms such as (119)-(123).
} 
guarantee that if the second derivatives of $D_{o}^{*}$ and $g_{r}$ are the same, their first derivatives are also the same. Therefore, (109) and (110) imply (107) and (108). QED. 
Proof of Proposition 3: ${ }^{87}$ This proposition claims that even under the restrictions of (63)-(67), $h_{r}$ can approximate any arbitrary output distance function $D_{i}^{*}$ to the second order at $\left(x^{*}, y^{*}\right)$. Stated differently, the following equations are satisfied.

$$
\begin{gathered}
h_{r}\left(y^{*}, x^{*}\right)=D_{i}^{*}\left(y^{*}, x^{*}\right)=1, \\
\frac{\partial h_{r}\left(y^{*}, x^{*}\right)}{\partial x_{n}}=\frac{\partial D_{i}^{*}\left(y^{*}, x^{*}\right)}{\partial x_{n}}, \forall n \in[1, \ldots, N], \\
\frac{\partial h_{r}\left(y^{*}, x^{*}\right)}{\partial y_{m}}=\frac{\partial D_{i}^{*}\left(y^{*}, x^{*}\right)}{\partial y_{m}}, \forall m \in[1, \ldots, M], \\
\frac{\partial^{2} h_{r}\left(y^{*}, x^{*}\right)}{\partial x_{u} \partial x_{v}}=\frac{\partial^{2} D_{i}^{*}\left(y^{*}, x^{*}\right)}{\partial x_{u} \partial x_{v}}, \forall u, v \in[1, \ldots, N], \\
\frac{\partial^{2} h_{r}\left(y^{*}, x^{*}\right)}{\partial y_{j} \partial y_{k}}=\frac{\partial^{2} D_{i}^{*}\left(y^{*}, x^{*}\right)}{\partial y_{j} \partial y_{k}}, \forall j, k \in[1, \ldots, M], \\
\frac{\partial^{2} h_{r}\left(y^{*}, x^{*}\right)}{\partial x_{n} \partial y_{m}}=\frac{\partial^{2} D_{i}^{*}\left(y^{*}, x^{*}\right)}{\partial x_{n} \partial y_{m}}, \forall n \in[1, \ldots, N], \forall m \in[1, \ldots, M] .
\end{gathered}
$$

We define the parameters in $g_{r}$ as follows: ${ }^{88}$

$$
\begin{gathered}
a_{j, k}=y_{j}^{* 1-r / 2} y_{k}^{* 1-r / 2}\left[-\frac{\partial^{2} D_{i}^{*}\left(y^{*}, x^{*}\right)}{\partial y_{j} \partial y_{k}}+(1+r) \frac{\partial D_{i}^{*}\left(y^{*}, x^{*}\right)}{\partial y_{j}} \frac{\partial D_{i}^{*}\left(y^{*}, x^{*}\right)}{\partial y_{k}}\right], \\
\forall j \neq k \in[1, \ldots, M], \\
a_{m, m}=y_{m}^{* 2-r}\left[-\frac{\partial^{2} D_{i}^{*}\left(y^{*}, x^{*}\right)}{\partial y_{j} \partial y_{k}}\right. \\
\left.+(1+r)\left(\frac{\partial D_{i}^{*}\left(y^{*}, x^{*}\right)}{\partial y_{m}}\right)^{2}-\left(1-\frac{r}{2}\right) \frac{\partial D_{i}^{*}\left(y^{*}, x^{*}\right)}{\partial y_{m}} \frac{1}{y_{m}^{*}}\right], \\
\forall m \in[1, \ldots, M], \\
c_{u, v}=x_{u}^{* 1-r / 2} x_{v}^{* 1-r / 2}\left[\frac{\partial^{2} D_{i}^{*}\left(y^{*}, x^{*}\right)}{\partial x_{u} \partial x_{v}}-(1-r) \frac{\partial D_{i}^{*}\left(y^{*}, x^{*}\right)}{\partial x_{u}} \frac{\partial D_{i}^{*}\left(y^{*}, x^{*}\right)}{\partial x_{v}}\right], \\
c_{n, n}=x_{n}^{* 2-r}\left[\frac{\partial^{2} D_{i}^{*}\left(y^{*}, x^{*}\right)}{\partial x_{n}^{2}}-(1-r)\left(\frac{\partial D_{i}^{*}\left(y^{*}, x^{*}\right)}{\partial x_{n}}\right)^{2}+\left(1-\frac{r}{2}\right) \frac{\partial D_{i}^{*}\left(y^{*}, x^{*}\right)}{\partial x_{n}} \frac{1}{x_{n}^{*}}\right], \\
\forall n \in[1, \ldots, N], \\
b_{m, n}=\frac{4}{r} \frac{y_{m}^{* 1+r / 2} x_{n}^{* 1-r / 2}}{\left(\sum_{j=1}^{M} \alpha_{j} y_{j}^{*-r / 2}\right)\left(\sum_{u=1}^{N} \beta_{u} x_{u}^{* r / 2}\right)}\left[-\frac{\partial^{2} D_{i}^{*}\left(y^{*}, x^{*}\right)}{\partial y_{m} \partial x_{n}}+\frac{\partial D_{i}^{*}\left(y^{*}, x^{*}\right)}{\partial y_{m}} \frac{\partial D_{i}^{*}\left(y^{*}, x^{*}\right)}{\partial x_{n}}\right], \\
\forall n \in[1, \ldots, N], \forall m \in[1, \ldots, M] .
\end{gathered}
$$

First, we show that these parameters specified by (130)-(134) satisfy the restrictions (64)-(67). By summing $a_{j, k} y_{k}^{* r / 2}$ over $k$, we can derive the following equation.

$$
\begin{aligned}
& \sum_{k=1}^{M} a_{j, k} y_{k}^{* r / 2}=\sum_{k \neq j} a_{j, k} y_{k}^{* r / 2}+a_{j, j} y_{j}^{* r / 2} \\
& =\sum_{k \neq j} y_{j}^{* 1-r / 2} y_{k}^{*}\left[-\frac{\partial^{2} D_{i}^{*}\left(y^{*}, x^{*}\right)}{\partial y_{j} \partial y_{k}}+(1+r) \frac{\partial D_{i}^{*}\left(y^{*}, x^{*}\right)}{\partial y_{j}} \frac{\partial D_{i}^{*}\left(y^{*}, x^{*}\right)}{\partial y_{k}}\right]+ \\
& y_{j}^{* 2-r / 2}\left[-\frac{\partial^{2} D_{i}^{*}\left(y^{*}, x^{*}\right)}{\partial y_{j}^{2}}+(1+r)\left(\frac{\partial D_{i}^{*}\left(y^{*}, x^{*}\right)}{\partial y_{j}}\right)^{2}-\left(1-\frac{r}{2}\right) \frac{\partial D_{i}^{*}\left(y^{*}, x^{*}\right)}{\partial y_{j}} \frac{1}{y_{j}^{*}}\right] \\
& \text { from (130) and (131), }
\end{aligned}
$$

\footnotetext{
${ }^{87}$ This proof is similar to the proof of Proposition 1. We provide it for completeness.

${ }^{88}$ This specification of parameters is just an example. There might exist an alternative specification which guarantees the second order approximation property. All the specifications are reduced to those adopted by Diewert (1992) for $r=2$, except for (134). Equation (87) in page 233 of Diewert (1992) indicates $b_{m, n}=\frac{y_{m}^{*}}{\left(\sum_{j=1}^{M} \alpha_{j} y_{j}^{*-1}\right)\left(\Sigma_{u=1}^{N} \beta_{u} x_{u}^{* 1}\right)}\left[-\frac{\partial^{2} D_{i}^{*}\left(x^{*}, y^{*}\right)}{\partial y_{m} \partial x_{n}}+\frac{\partial D_{i}^{*}\left(x^{*}, y^{*}\right)}{\partial y_{m}} \frac{\partial D_{i}^{*}\left(x^{*}, y^{*}\right)}{\partial x_{n}}\right]$ for $m=1, \ldots, M$ and $n=$ $1, \ldots, N$. It is not true. The right hand side of the equation needs to be multiplied by two as (134) suggests for the case $r=2$.
} 


$$
\begin{aligned}
& =y_{j}^{* 1-r / 2} \sum_{k=1}^{M}\left[-\frac{\partial^{2} D_{i}^{*}\left(y^{*}, x^{*}\right)}{\partial y_{j} \partial y_{k}} y_{k}^{*}+(1+r) \frac{\partial D_{i}^{*}\left(y^{*}, x^{*}\right)}{\partial y_{j}} \frac{\partial D_{i}^{*}\left(y^{*}, x^{*}\right)}{\partial y_{k}} y_{k}^{*}\right]- \\
& \left(1-\frac{r}{2}\right) y_{j}^{* 1-r / 2} \frac{\partial D_{i}^{*}\left(y^{*}, x^{*}\right)}{\partial y_{j}} \\
& =\left(-(-2)-(1+r)-\left(1-\frac{r}{2}\right)\right) y_{j}^{* 1-r / 2} \frac{\partial D_{i}^{*}\left(y^{*}, x^{*}\right)}{\partial y_{j}} \\
& \text { from }(17),(23) \text { and } \\
& =-\left(\frac{r}{2}\right) y_{j}^{* 1-r / 2} \frac{\partial D_{i}^{*}\left(y^{*}, x^{*}\right)}{\partial y_{j}} .
\end{aligned}
$$

Then, it implies (64) as follows:

$$
\begin{aligned}
& \sum_{j=1}^{M} \sum_{k=1}^{M} a_{j, k} y_{j}^{* r / 2} y_{k}^{* r / 2}=-\sum_{j=1}^{M} y_{j}^{* r / 2}\left(\frac{r}{2}\right) y_{j}^{* 1-r / 2} \frac{\partial D_{i}^{*}\left(y^{*}, x^{*}\right)}{\partial y_{j}} \\
& =-\sum_{j=1}^{M}\left(\frac{r}{2}\right) y_{j}^{*} \frac{\partial D_{i}^{*}\left(y^{*}, x^{*}\right)}{\partial y_{j}}=\frac{r}{2} D_{i}^{*}\left(y^{*}, x^{*}\right)=\frac{r}{2}
\end{aligned}
$$

from (17) and (23).

By summing $c_{u, v} x_{v}^{* r / 2}$ over $v$, we can derive the following equation.

$$
\begin{aligned}
& \sum_{v=1}^{N} c_{u, v} x_{v}^{* r / 2}=\sum_{v \neq u} c_{u, v} x_{v}^{* r / 2}+c_{u, u} x_{u}^{* r / 2} \\
& =\sum_{v \neq u} x_{u}^{* 1-r / 2} x_{v}^{*}\left[\frac{\partial^{2} D_{i}^{*}\left(y^{*}, x^{*}\right)}{\partial x_{u} \partial x_{v}}-(1-r) \frac{\partial D_{i}^{*}\left(y^{*}, x^{*}\right)}{\partial x_{u}} \frac{\partial D_{i}^{*}\left(y^{*}, x^{*}\right)}{\partial x_{v}}\right]+ \\
& x_{u}^{* 2-r / 2}\left[\frac{\partial^{2} D_{i}^{*}\left(y^{*}, x^{*}\right)}{\partial x_{u}^{2}}-(1-r)\left(\frac{\partial D_{i}^{*}\left(y^{*}, x^{*}\right)}{\partial x_{u}}\right)^{2}+\left(1-\frac{r}{2}\right) \frac{\partial D_{i}^{*}\left(y^{*}, x^{*}\right)}{\partial x_{u}} \frac{1}{x_{u}^{*}}\right] \\
& \text { from }(132) \text { and (133), } \\
& =x_{u}^{* 1-r / 2} \sum_{v=1}^{N}\left[\frac{\partial^{2} D_{i}^{*}\left(y^{*}, x^{*}\right)}{\partial x_{u} \partial x_{v}} x_{v}^{*}-(1-r) \frac{\partial D_{i}^{*}\left(y^{*}, x^{*}\right)}{\partial x_{u}} \frac{\partial D_{i}^{*}\left(y^{*}, x^{*}\right)}{\partial x_{v}} x_{v}^{*}\right]+ \\
& \left(1-\frac{r}{2}\right) x_{u}^{* 1-r / 2} \frac{\partial D_{i}^{*}\left(y^{*}, x^{*}\right)}{\partial x_{u}} \\
& =\left(-(1-r)+\left(1-\frac{r}{2}\right)\right) x_{u}^{* 1-r / 2} \frac{\partial D_{i}^{*}\left(y^{*}, x^{*}\right)}{\partial x_{u}} \\
& =\left(\frac{r}{2}\right) x_{u}^{* 1-r / 2} \frac{\partial D_{i}^{*}\left(y^{*}, x^{*}\right)}{\partial x_{u}} .
\end{aligned}
$$

Then, it implies (65) as follows:

$$
\begin{aligned}
& \sum_{u=1}^{N} \sum_{v=1}^{N} c_{u, v} x_{u}^{* r / 2} x_{v}^{* r / 2}=\sum_{u=1}^{N} x_{u}^{* r / 2}\left(\frac{r}{2}\right) x_{u}^{* 1-r / 2} \frac{\partial D_{i}^{*}\left(y^{*}, x^{*}\right)}{\partial x_{u}} \\
& =\sum_{u=1}^{N}\left(\frac{r}{2}\right) x_{u}^{*} \frac{\partial D_{i}^{*}\left(y^{*}, x^{*}\right)}{\partial x_{u}}=\frac{r}{2} D_{i}^{*}\left(x^{*}, y^{*}\right)=\frac{r}{2}
\end{aligned}
$$

from (17) and (20).

By summing $b_{m, n} y_{m}^{*}{ }^{-r / 2}$ over $m$, we obtain (66) so as

$$
\begin{aligned}
& \sum_{m=1}^{M} b_{m, n} y_{m}^{*-r / 2}=\frac{4}{r} \frac{x_{n}^{* 1-r / 2}}{\left(\sum_{j=1}^{M} \alpha_{j} y_{j}^{*-r / 2}\right)\left(\sum_{u=1}^{N} \beta_{u} x_{u}^{* r / 2}\right)} \times \\
& \sum_{m=1}^{M}\left[-\frac{\partial^{2} D_{i}^{*}\left(y^{*}, x^{*}\right)}{\partial y_{m} \partial x_{n}} y_{m}^{*}+\frac{\partial D_{i}^{*}\left(y^{*}, x^{*}\right)}{\partial y_{m}} y_{m}^{*} \frac{\partial D_{i}^{*}\left(y^{*}, x^{*}\right)}{\partial x_{n}}\right] \\
& =\frac{4}{r} \frac{x_{n}^{* 1-r / 2}}{\left(\sum_{j=1}^{M} \alpha_{j} y_{j}^{*-r / 2}\right)\left(\sum_{u=1}^{N} \beta_{u} x_{u}^{* r / 2}\right)} \times(1-1) \frac{\partial D_{i}^{*}\left(y^{*}, x^{*}\right)}{\partial x_{n}}=0
\end{aligned}
$$

from (17), (23) and (25).

By summing $b_{m, n} x_{n}^{* r / 2}$ over $n$, we obtain (67) so as 


$$
\begin{aligned}
& \sum_{n=1}^{N} b_{m, n} x_{n}^{* r / 2}=\frac{4}{r} \frac{y_{m}^{*}{ }^{1+r / 2}}{\left(\sum_{m=1}^{M} \alpha_{m} y_{m}^{*}{ }^{-r / 2}\right)\left(\sum_{u=1}^{N} \beta_{u} x_{u}^{* r / 2}\right)} \times \\
& \sum_{n=1}^{N}\left[-\frac{\partial^{2} D_{i}^{*}\left(y^{*}, x^{*}\right)}{\partial y_{m} \partial x_{n}} x_{n}^{*}+\frac{\partial D_{i}^{*}\left(y^{*}, x^{*}\right)}{\partial y_{m}} \frac{\partial D_{i}^{*}\left(y^{*}, x^{*}\right)}{\partial x_{n}} x_{n}^{*}\right] \\
& =\frac{4}{r} \frac{y_{m}^{*}{ }^{1+r / 2}}{\left(\sum_{m=1}^{M} \alpha_{m} y_{m}^{*}{ }^{-r / 2}\right)\left(\sum_{u=1}^{N} \beta_{u} x_{u}^{*}{ }^{* / 2}\right)} \times(-1+1) \frac{\partial D_{i}^{*}\left(y^{*}, x^{*}\right)}{\partial y_{m}}=0 \\
& \text { from (17), (20) and (22). }
\end{aligned}
$$

Second, we show that these parameters specified by (130)-(134) satisfy the equations for the second order approximation (124)-(129).

As we show, (130)-(134) imply (64)-(67). Substituting (64)-(67) into (60), we obtain $h_{r}\left(y^{*}, x^{*}\right)=[(r / 2) /(r / 2)]^{1 / r}=1$, which implies (124) along with (17).

The second-order derivatives of $h_{r}$ with respect to inputs $x$ evaluated at $\left(y^{*}, x^{*}\right)$ are given by the following equations, using (64)-(67). ${ }^{89}$

$$
\begin{gathered}
\frac{\partial^{2} h_{r}\left(y^{*}, x^{*}\right)}{\partial x_{u} \partial x_{v}}=(1-r)\left(\frac{r}{2}\right)^{-2} \times \\
x_{u}^{* r / 2-1} x_{v}^{* r / 2-1}\left(\sum_{n=1}^{N} c_{u, n} x_{n}^{* r / 2}\right)\left(\sum_{n=1}^{N} c_{v, n} x_{n}^{* r / 2}\right) \\
-c_{u, v} x_{u}^{* r / 2-1} x_{v}^{* r / 2-1}, \quad \forall u \neq v \in[1, \ldots, N], \\
\frac{\partial^{2} h_{r}\left(y^{*}, x^{*}\right)}{\partial x_{n}^{2}}=(1-r)\left(\frac{r}{2}\right)^{-2} \times x_{n}^{* r-2}\left(\sum_{u=1}^{N} c_{n, u} x_{u}^{* r / 2}\right)^{2} \\
-c_{n, n} x_{n}^{* r-2}-\left(1-\frac{r}{2}\right)\left(\frac{r}{2}\right)^{-1} x_{n}^{* r / 2-2}\left(\sum_{u=1}^{N} c_{n, u} x_{u}^{* r / 2}\right), \\
\forall n \in[1, \ldots, N] .
\end{gathered}
$$

Substituting (136) into (137) and (138), we obtain (127).

The second-order derivatives of $h_{r}$ with respect to outputs $y$ evaluated at $\left(y^{*}, x^{*}\right)$ are given by the following equations, using (64)-(67).

$$
\begin{gathered}
\frac{\partial^{2} h_{r}\left(y^{*}, x^{*}\right)}{\partial y_{j} \partial y_{k}}=\left((1-r)\left(\frac{r}{2}\right)^{-2}+4\left(\frac{r}{2}\right)^{-1}\right) \\
\times y_{j}^{* r / 2-1} y_{k}^{* r / 2-1}\left(\sum_{m=1}^{M} a_{j, m} y_{m}^{* r / 2}\right)\left(\sum_{m=1}^{M} a_{k, m} y_{m}^{* r / 2}\right)- \\
a_{j, k} y_{j}^{* r / 2-1} y_{k}^{* r / 2-1}, \\
\frac{\partial^{2} h_{r}\left(y^{*}, x^{*}\right)}{\partial y_{m}^{2}}=\left((1-r)\left(\frac{r}{2}\right)^{-2}+4\left(\frac{r}{2}\right)^{-1}\right) \\
\times y_{j}^{* r / 2-1} y_{k}^{* r / 2-1}\left(\sum_{m=1}^{M} a_{j, m} y_{m}^{* r / 2}\right)\left(\sum_{m=1}^{M} a_{k, m} y_{m}^{* r / 2}\right)- \\
a_{j, k} y_{j}^{* r / 2-1} y_{k}^{* r / 2-1}+\left(1-\frac{r}{2}\right)\left(\frac{r}{2}\right)^{-1} y_{m}^{* r / 2-2}\left(\sum_{j=1}^{M} a_{m, j} y_{j}^{* r / 2}\right), \\
\forall m \in[1, \ldots, M],
\end{gathered}
$$

Substituting (135) into (139) and (140), we obtain (128).

\footnotetext{
${ }^{89}$ Without any restrictions on the parameters on $g_{r}$, its second derivatives are too complicated to be written down explicitly here. The restrictions by (64)-(67) cause their representation to be in much simpler forms such as (137)-(141).
} 
The second-order cross-derivatives of $g_{r}$ with respect to inputs $x$ and outputs $y$ evaluated at $\left(y^{*}, x^{*}\right)$ are given by the following equation, using (66) and (67).

$$
\begin{aligned}
& \frac{\partial^{2} h_{r}\left(y^{*}, x^{*}\right)}{\partial y_{m} \partial x_{n}} \\
& =-\left(\frac{r}{2}\right)^{-2} y_{m}^{* r / 2-1} x_{n}^{* r / 2-1}\left(\sum_{j=1}^{M} a_{m, j} y_{j}^{* r / 2}\right)\left(\sum_{u=1}^{N} c_{n, u} x_{u}^{* r / 2}\right) \\
& \quad-\left(\frac{r}{4}\right)\left(\sum_{j=1}^{M} \alpha_{j} y_{j}^{* \frac{r}{2}}\right)\left(\sum_{u=1}^{N} \beta_{u} x_{u}^{*}{ }^{-\frac{r}{2}}\right) b_{m, n} y_{m}^{* r / 2-1} x_{n}^{*-r / 2-1}, \\
& \forall n \in[1, \ldots, N], \forall m \in[1, \ldots, M] .
\end{aligned}
$$

Substituting (134)-(136) into (141), we obtain (129).

Since $D_{i}^{*}$ and $h_{r}$ are linearly homogeneous in inputs $x$ and homogeneous of degree minus one in outputs $y$, (20)-(25) hold for both of them. Equations (22), (24) and (25) guarantee that if the second derivatives of $D_{i}^{*}$ and $h_{r}$ are the same, their first derivatives are also the same. Therefore, (127) and (128) imply (125) and (126). QED. 
Proof of Proposition 4: The revenue maximization implies (7). Substituting either (7), (74) and (75) or (7), (76) and (77) into (71), we obtain the following equation:

$$
\sigma^{t}\left(\sum_{j=1}^{M} \sum_{k=1}^{M} a_{j, k} y_{j}^{t^{r / 2}} y_{k}^{t^{r / 2}}\right)^{1 / r}\left(\sum_{u=1}^{N} \sum_{v=1}^{N} c_{u, v}^{t} x_{u}^{t^{r / 2}} x_{v}^{t^{r / 2}}\right)^{-1 / r}=1
$$

The first-order derivatives of $g_{r}^{t}$ with respect to output $y_{m}$ are given by the following equations for $t=0,1$, using (74) or (77) for $g_{r}^{0}$ and (75) and (76) for $g_{r}^{1}$ along with (142) for both $g_{r}^{0}$ and $g_{r}^{1}$ :

$$
\begin{aligned}
& \frac{\partial D_{o}^{t}\left(x^{t}, y^{t}\right)}{\partial y_{m}}=\frac{\partial g_{r}^{t}\left(x^{t}, y^{t}\right)}{\partial y_{m}} \\
& =\left(\sigma^{t}\right)^{r}\left(\sum_{u=1}^{N} \sum_{v=1}^{N} c_{u, v}^{t} x_{u}^{t^{r / 2}} x_{v}^{t^{r / 2}}\right)^{-1} \times \sum_{k=1}^{M} a_{m, k} y_{m}^{t}{ }^{r / 2-1} y_{k}^{t r / 2}, \\
& \forall m \in[1, \ldots, M] .
\end{aligned}
$$

Substituting (143) into (70), we obtain the following equations:

$$
\begin{gathered}
\left(\frac{p_{m}^{0} y_{m}^{0}}{\sum_{j=1}^{M} p_{j}^{0} y_{j}^{0}}\right)\left(\frac{y_{m}^{1}}{y_{m}^{0}}\right)^{r / 2} \\
=\left(\sigma^{0}\right)^{r}\left(\sum_{u=1}^{N} \sum_{v=1}^{N} c_{u, v}^{0} x_{u}^{0^{r / 2}} x_{v}^{0^{r / 2}}\right)^{-1} \times \sum_{k=1}^{M} a_{m, k} y_{m}^{1}{ }^{r / 2} y_{k}^{0^{r / 2}}, \\
\forall m \in[1, \ldots, M], \\
\left(\frac{p_{m}^{1} y_{m}^{1}}{\sum_{j=1}^{M} p_{j}^{1} y_{j}^{1}}\right)\left(\frac{y_{m}^{0}}{y_{m}^{1}}\right)^{r / 2} \\
=\left(\sigma^{1}\right)^{r}\left(\sum_{u=1}^{N} \sum_{v=1}^{N} c_{u, v}^{1} x_{u}^{1}{ }^{r / 2} x_{v}^{1^{r / 2}}\right)^{-1} \times \sum_{k=1}^{M} a_{m, k} y_{m}^{0^{r / 2}} y_{k}^{1}{ }^{r / 2} \\
\forall m \in[1, \ldots, M] .
\end{gathered}
$$

Substituting (144) and (145) into (34), we obtain the following equation using (142):

$$
\begin{gathered}
Q M O I_{r}=\frac{\sigma^{0}\left(\sum_{u=1}^{N} \sum_{v=1}^{N} c_{u, v}^{0} x_{u}^{0^{r / 2}} x_{v}^{0^{r / 2}}\right)^{-1 / r}\left(\sum_{j=1}^{M} \sum_{k=1}^{M} a_{j, k} y_{j}^{1}{ }^{r / 2} y_{k}^{0^{r / 2}}\right)^{1 / r}}{\sigma^{1}\left(\sum_{u=1}^{N} \sum_{v=1}^{N} c_{u, v}^{1} x_{u}^{1}{ }^{r / 2} x_{v}^{1}{ }^{r / 2}\right)^{-1 / r}\left(\sum_{j=1}^{M} \sum_{k=1}^{M} a_{j, k} y_{j}^{{ }^{r / 2}} y_{k}^{1}{ }^{r / 2}\right)^{1 / r}} \\
\Leftrightarrow Q M O I_{r}=\frac{\left(\sum_{j=1}^{M} \sum_{k=1}^{M} a_{j, k} y_{j}^{1}{ }^{r / 2} y_{k}^{1}{ }^{r / 2}\right)^{1 / r}}{\left(\sum_{j=1}^{M} \sum_{k=1}^{M} a_{j, k} y_{j}^{0^{r / 2}} y_{k}^{0^{r / 2}}\right)^{1 / r} .}
\end{gathered}
$$

Substituting (71) into $M O I^{0}$ and $M O I^{1}$ defined by (26) and using either (74), (75) and (142) or (76), (77) and (142) we obtain the following equation:

$$
M O I^{0}=M O I^{1}=\frac{\left(\sum_{j=1}^{M} \sum_{k=1}^{M} a_{j, k} y_{j}^{1}{ }^{r / 2} y_{k}^{1}{ }^{r / 2}\right)^{1 / r}}{\left(\sum_{j=1}^{M} \sum_{k=1}^{M} a_{j, k} y_{j}^{0^{r / 2}} y_{k}^{0^{r / 2}}\right)^{1 / r}} .
$$

Thus, (146) and (147) implies the following equation.

$$
Q M O I_{r}=M O I^{0}=M O I^{1}=M O I .
$$

QED. 
Proof of Proposition 5: The cost minimization implies (17). Substituting either (17), (83) and (84) or (17), (85) and (86) into (80), we obtain the following equation:

$$
\begin{array}{r}
\sigma^{t}\left(\sum_{j=1}^{M} \sum_{k=1}^{M} a_{j, k}^{t} y_{j}^{t^{r / 2}} y_{k}^{t^{r / 2}}\right)^{-1 / r}\left(\sum_{u=1}^{N} \sum_{v=1}^{N} c_{u, v} x_{u}^{t^{r / 2}} x_{v}^{t^{r / 2}}\right)^{1 / r}=1, \\
\text { for } t=0,1 .
\end{array}
$$

The first-order derivatives of $h_{r}^{t}$ with respect to input $x_{n}$ are given by the following equations for $t=0,1$, using (83) or (86) for $h_{r}^{0}$ and (84) or (85) for $h_{r}^{1}$ along with (149) for both $h_{r}^{0}$ and $h_{r}^{1}$ :

$$
\begin{aligned}
& \frac{\partial D_{i}^{t}\left(y^{t}, x^{t}\right)}{\partial x_{n}}=\frac{\partial h_{r}^{t}\left(y^{t}, x^{t}\right)}{\partial x_{m}} \\
& \quad=\left(\sigma^{t}\right)^{r}\left(\sum_{j=1}^{M} \sum_{k=1}^{M} a_{j, k}^{t} y_{j}^{t^{r / 2}} y_{k}^{t^{r / 2}}\right)^{-1} \sum_{v=1}^{N} c_{n, v} x_{n}^{t^{r / 2-1}} x_{v}^{t^{r / 2}}, \\
& \quad \forall n \in[1, \ldots, N] .
\end{aligned}
$$

Substituting (150) into (79), we obtain the following equations:

$$
\begin{gathered}
\left(\frac{w_{n}^{0} x_{n}^{0}}{\sum_{u=1}^{N} w_{u}^{0} x_{u}^{0}}\right)\left(\frac{x_{n}^{1}}{x_{n}^{0}}\right)^{r / 2} \\
=\left(\sigma^{0}\right)^{r}\left(\sum_{j=1}^{M} \sum_{k=1}^{M} a_{j, k}^{0} y_{j}^{0^{r / 2}} y_{k}^{0^{r / 2}}\right)^{-1} \sum_{v=1}^{N} c_{n, v} x_{n}^{1}{ }^{r / 2} x_{v}^{0^{r / 2}}, \\
\forall n \in[1, \ldots, N] . \\
\left(\frac{w_{n}^{1} x_{n}^{1}}{\sum_{u=1}^{N} w_{u}^{1} x_{u}^{1}}\right)\left(\frac{x_{n}^{1}}{x_{n}^{0}}\right)^{r / 2} \\
=\left(\sigma^{1}\right)^{r}\left(\sum_{j=1}^{M} \sum_{k=1}^{M} a_{j, k}^{1} y_{j}^{1}{ }^{r / 2} y_{k}^{1}\right)^{r / 2} \sum_{v=1}^{N} c_{n, v} x_{n}{ }^{r / 2} x_{v}^{1}{ }^{r / 2}, \\
\forall n \in[1, \ldots, N] .
\end{gathered}
$$

Substituting (151) and (152) into (40), we obtain the following equation using (149):

$$
\begin{gathered}
Q M I I_{r}=\frac{\sigma^{0}\left(\sum_{j=1}^{M} \sum_{k=1}^{M} a_{j, k}^{0} y_{j}^{0^{r / 2}} y_{k}^{y^{r / 2}}\right)^{-1 / r}\left(\sum_{u=1}^{N} \sum_{v=1}^{N} c_{u, v} x_{u}^{1 / 2} x_{v}^{0^{r / 2}}\right)^{1 / r}}{\left.\sigma^{1}\left(\sum_{j=1}^{M} \sum_{k=1}^{M} a_{j, k}^{1} y_{j}^{1}{ }^{r / 2} y_{k}^{1}\right)^{r / 2}\right)^{-1 / r}\left(\sum_{u=1}^{N} \sum_{v=1}^{N} c_{u, v} x_{u}^{r / 2} x_{v}^{1 / 2}\right)^{1 / r}} \\
\Leftrightarrow Q M I I_{r}=\frac{\left(\sum_{u=1}^{N} \sum_{v=1}^{N} c_{u, v} x_{u}^{1} r / 2 x_{v}^{1}{ }^{r / 2}\right)^{1 / r}}{\left(\sum_{u=1}^{N} \sum_{v=1}^{N} c_{u, v} x_{u}^{0^{r / 2}} x_{v}^{0^{r / 2}}\right)^{1 / r}}
\end{gathered}
$$

Substituting (80) into $M I I^{0}$ and $M I I^{1}$ defined by (27) and using either (83), (84) and (149) or (85), (86) and (149), we obtain the following equation:

$$
M I I^{0}=M I^{1}=\frac{\left(\sum_{u=1}^{N} \sum_{v=1}^{N} c_{u, v} x_{u}^{1}{ }^{r / 2} x_{v}^{1}{ }^{r / 2}\right)^{1 / r}}{\left(\sum_{u=1}^{N} \sum_{v=1}^{N} c_{u, v} x_{u}^{0^{r / 2}} x_{v}^{0^{r / 2}}\right)^{1 / r}} .
$$

Thus, (153) and (154) implies the following equation:

$$
Q M I I_{r}=M I I^{0}=M I I^{1}=M I I .
$$

QED. 
Proof of Proposition 6: The profit maximization implies (7). Substituting either (7) and (94)-(99) into (91) or (7) and (100)-(105) into (91), we obtain the following equation:

$$
\begin{gathered}
\sigma^{t}\left(\sum_{j=1}^{M} \sum_{k=1}^{M} a_{j, k} y_{j}^{t^{r / 2}} y_{k}^{t^{r / 2}}\right)^{1 / r}\left(\sum_{u=1}^{N} \sum_{v=1}^{N} c_{u, v} x_{u}^{t^{r / 2}} x_{v}^{t^{r / 2}}\right)^{-1 / r}=1 \\
\text { for } t=0,1 .
\end{gathered}
$$

The first-order derivatives of $g_{r}^{t}$ with respect to output $y_{m}$ are given by the following equations for $t=0,1$, using (94) or (104) for $\hat{g}_{r}^{0}$ and (98) or (100) for $\hat{g}_{r}^{1}$ along with (156) for both $\hat{g}_{r}^{0}$ and $\hat{g}_{r}^{1}$ :

$$
\begin{aligned}
& \frac{\partial D_{o}^{t}\left(x^{t}, y^{t}\right)}{\partial y_{m}}=\frac{\partial \hat{g}_{r}^{t}\left(x^{t}, y^{t}\right)}{\partial y_{m}} \\
& =\left(\sigma^{t}\right)^{r}\left(\sum_{u=1}^{N} \sum_{v=1}^{N} c_{u, v} x_{u}^{t^{r / 2}} x_{v}^{t r / 2}\right)^{-1} \times \sum_{k=1}^{M} a_{m, k} y_{m}^{t}{ }^{r / 2-1} y_{k}^{t r / 2}, \\
& \forall m \in[1, \ldots, M] .
\end{aligned}
$$

Substituting (157) into (88), we obtain the following equations:

$$
\begin{gathered}
\left(\frac{p_{m}^{0} y_{m}^{0}}{\sum_{j=1}^{M} p_{j}^{0} y_{j}^{0}}\right)\left(\frac{y_{m}^{1}}{y_{m}^{0}}\right)^{r / 2} \\
=\left(\sigma^{0}\right)^{r}\left(\sum_{u=1}^{N} \sum_{v=1}^{N} c_{u, v} x_{u}^{0^{r / 2}} x_{v}^{0^{r / 2}}\right)^{-1} \times \sum_{k=1}^{M} a_{m, k} y_{m}^{1} r / 2 y_{k}^{0^{r / 2}}, \\
\forall m \in[1, \ldots, M], \\
\left(\frac{p_{m}^{1} y_{m}^{1}}{\sum_{j=1}^{M} p_{j}^{1} y_{j}^{1}}\right)\left(\frac{y_{m}^{0}}{y_{m}^{1}}\right)^{r / 2} \\
=\left(\sigma^{1}\right)^{r}\left(\sum_{u=1}^{N} \sum_{v=1}^{N} c_{u, v} x_{u}^{1}{ }^{r / 2} x_{v}^{1}{ }^{r / 2}\right)^{-1} \times \sum_{k=1}^{M} a_{m, k} y_{m}^{0} r / 2 y_{k}^{1} r / 2 \\
\forall m \in[1, \ldots, M] .
\end{gathered}
$$

The first-order derivatives of $g_{r}^{t}$ with respect to input $x_{n}$ are given by the following equations for $t=0,1$, using (95) or (103) for $\hat{g}_{r}^{0}$ and (97) or (101) for $\hat{g}_{r}^{1}$ along with (156) for both $\hat{g}_{r}^{0}$ and $\hat{g}_{r}^{1}$ :

$$
\begin{aligned}
& \frac{\partial D_{o}^{t}\left(x^{t}, y^{t}\right)}{\partial x_{n}}=\frac{\partial \hat{g}_{r}^{t}\left(x^{t}, y^{t}\right)}{\partial x_{n}} \\
& =-\left(\sigma^{t}\right)^{r}\left(\sum_{j=1}^{M} \sum_{k=1}^{M} a_{j, k} y_{j}^{t^{r / 2}} y_{k}^{t^{r / 2}}\right)\left(\sum_{u=1}^{N} \sum_{v=1}^{N} c_{u, v} x_{u}^{t^{\frac{r}{2}}} x_{v}^{t^{\frac{r}{2}}}\right)^{-2} \times \\
& \begin{array}{ll}
\sum_{v=1}^{N} c_{n, v} x_{n}^{t^{r / 2-1}} x_{v}^{t^{r / 2}}, & \forall n \in[1, \ldots, N] .
\end{array}
\end{aligned}
$$

Substituting (160) into (89), we obtain the following equations:

$$
\begin{aligned}
& \left(\frac{w_{n}^{0} x_{n}^{0}}{\sum_{u=1}^{N} w_{u}^{0} x_{u}^{0}}\right)\left(\frac{x_{n}^{1}}{x_{n}^{0}}\right)^{r / 2} \\
& =-\left(\sigma^{0}\right)^{r}\left(\sum_{j=1}^{M} \sum_{k=1}^{M} a_{j, k} y_{j}^{0^{r / 2}} y_{k}^{0^{r / 2}}\right)\left(\sum_{u=1}^{N} \sum_{v=1}^{N} c_{u, v} x_{u}^{0^{\frac{r}{2}}} x_{v}^{0^{\frac{r}{2}}}\right)^{-2} \times \\
& \sum_{v=1}^{N} c_{n, v} x_{n}^{1^{r / 2}} x_{v}^{0^{r / 2}} \text {, } \\
& \left(\frac{w_{n}^{1} x_{n}^{1}}{\sum_{u=1}^{N} w_{u}^{1} x_{u}^{1}}\right)\left(\frac{x_{n}^{0}}{x_{n}^{1}}\right)^{r / 2} \\
& =-\left(\sigma^{1}\right)^{r}\left(\sum_{j=1}^{M} \sum_{k=1}^{M} a_{j, k} y_{j}^{1}{ }^{r / 2} y_{k}^{1 r / 2}\right)\left(\sum_{u=1}^{N} \sum_{v=1}^{N} c_{u, v} x_{u}^{1^{\frac{r}{2}}} x_{v}^{1 \frac{r}{2}}\right)^{-2} \times \\
& \sum_{v=1}^{N} c_{n, v} x_{n}^{0^{r / 2}} x_{v}^{r / 2} \text {, } \\
& \forall n \in[1, \ldots, N]
\end{aligned}
$$


Substituting (158), (159), (161) and (162) into (46), we obtain the following equation using (156):

$$
\begin{aligned}
& Q M P I_{r}=\left(\frac{\sigma^{0}}{\sigma^{1}} \frac{\left(\sum_{u=1}^{N} \sum_{v=1}^{N} c_{u, v} x_{u}^{0^{r / 2}} x_{v}^{0^{r / 2}}\right)^{-1 / r}}{\left(\sum_{u=1}^{N} \sum_{v=1}^{N} c_{u, v} x_{u}^{1}{ }^{r / 2} x_{v}^{1}{ }^{r / 2}\right)^{-1 / r}} \frac{\left(\sum_{j=1}^{M} \sum_{k=1}^{M} a_{j, k} y_{j}^{1 r / 2} y_{k}^{0^{r / 2}}\right)^{1 / r}}{\left(\sum_{j=1}^{M} \sum_{k=1}^{M} a_{j, k} y_{j}^{0^{r / 2}} y_{k}^{1}{ }^{r / 2}\right)^{1 / r}}\right) \times \\
& \left(\frac{\sigma^{0}}{\sigma^{1}} \frac{\left(\sum_{j=1}^{M} \sum_{k=1}^{M} a_{j, k} y_{j}^{0^{r / 2}} y_{k}^{0^{r / 2}}\right)^{1 / r}}{\left(\sum_{j=1}^{M} \sum_{k=1}^{M} a_{j, k} y_{j}^{1 / 2}{y_{k}^{1}}^{r / 2}\right)^{1 / r}} \frac{\left(\sum_{u=1}^{N} \sum_{v=1}^{N} c_{u, v} x_{u}^{0^{r / 2}} x_{v}^{0^{r / 2}}\right)^{-2 / r}}{\left(\sum_{u=1}^{N} \sum_{v=1}^{N} c_{u, v} x_{u}^{1}{ }^{r / 2} x_{v}^{1}{ }^{r / 2}\right)^{-2 / r}}\right. \\
& \left.\times \frac{\left(\sum_{u=1}^{N} \sum_{v=1}^{N} c_{u, v} x_{u}^{1}{ }^{r / 2} x_{v}^{0}{ }^{r / 2}\right)^{1 / r}}{\left(\sum_{u=1}^{N} \sum_{v=1}^{N} c_{u, v} x_{u}^{0^{r / 2}} x_{v}^{1 r / 2}\right)^{1 / r}}\right)^{-1} \\
& \Leftrightarrow Q M P I_{r}=\frac{\left(\sum_{j=1}^{M} \sum_{k=1}^{M} a_{j, k} y_{j}^{0^{r / 2}} y_{k}^{0^{r / 2}}\right)^{-1 / r}}{\left(\sum_{j=1}^{M} \sum_{k=1}^{M} a_{j, k} y_{j}^{1 / 2} y_{k}^{1 r / 2}\right)^{-1 / r}} \frac{\left(\sum_{u=1}^{N} \sum_{v=1}^{N} c_{u, v} x_{u}^{0^{r / 2}} x_{v}^{0^{r / 2}}\right)^{1 / r}}{\left(\sum_{u=1}^{N} \sum_{v=1}^{N} c_{u, v} x_{u}^{1}{ }^{r / 2} x_{v}^{1 r / 2}\right)^{1 / r}} \\
& \Leftrightarrow Q M P I_{r}=\sigma^{0} / \sigma^{1} .
\end{aligned}
$$

Substituting (91) into $M P I^{0}$ and $M P I^{1}$ defined by (28) and using either (94)-(99) and (156) or (100)-(105) and (156), we obtain the following equation:

$$
M P I^{0}=M P I^{1}=\sigma^{1} / \sigma^{0} .
$$

Thus, (163) and (164) implies the following equation:

$$
Q M P I_{r}=M P I^{0}=M P I^{1}=M P I .
$$

QED. 
Proof of Corollary 1: First, we substitute (146) into the following equation:

$\frac{\left(\sum_{j=1}^{M} \sum_{k=1}^{M} a_{j, k} y_{j}^{2 r / 2} y_{k}^{2^{r / 2}}\right)^{1 / r}}{\left(\sum_{j=1}^{M} \sum_{k=1}^{M} a_{j, k} y_{j}^{0^{r / 2}}{y_{k}^{0^{r / 2}}}^{1 / r}\right.}=\frac{\left(\sum_{j=1}^{M} \sum_{k=1}^{M} a_{j, k} y_{j}^{1}{ }^{r / 2} y_{k}^{1 / 2}\right)^{1 / r}}{\left(\sum_{j=1}^{M} \sum_{k=1}^{M} a_{j, k}{y_{j}^{0}}^{r / 2} y_{k}^{0^{r / 2}}\right)^{1 / r}} \times \frac{\left(\sum_{j=1}^{M} \sum_{k=1}^{M} a_{j, k} y_{j}^{2^{r / 2}}{y_{k}^{2}}^{r / 2}\right)^{1 / r}}{\left(\sum_{j=1}^{M} \sum_{k=1}^{M} a_{j, k} y_{j}^{1^{r / 2}}{y_{k}^{1}}^{r / 2}\right)^{1 / r}}$.

Then, we obtain $Q M O I_{r}\left(p^{0}, p^{2}, y^{0}, y^{2}\right)=Q M O I_{r}\left(p^{0}, p^{1}, y^{0}, y^{1}\right) \times$ $Q M O I_{r}\left(p^{1}, p^{2}, y^{1}, y^{2}\right)$. Therefore, $Q M O I_{r}$ satisfies T21. QED.

Proof of Corollary 2: First, we substitute (153) into the following equation:

$\frac{\left(\sum_{u=1}^{N} \sum_{v=1}^{N} c_{u, v} x_{u}^{2}{ }^{r / 2} x_{v}^{2}{ }^{r / 2}\right)^{\frac{1}{r}}}{\left(\sum_{u=1}^{N} \sum_{v=1}^{N} c_{u, v} x_{u}^{0}{ }^{r / 2} x_{v}^{0}{ }^{r / 2}\right)^{\frac{1}{r}}}=\frac{\left(\sum_{u=1}^{N} \sum_{v=1}^{N} c_{u, v} x_{u}^{1 / 2} x_{v}^{r / 2}\right)^{1 / r}}{\left(\sum_{u=1}^{N} \sum_{v=1}^{N} c_{u, v} x_{u}^{0 / 2} x_{v}^{0}\right)^{r / 2}} \times \frac{\left(\sum_{u=1}^{N} \sum_{v=1}^{N} c_{u, v} x_{u}^{2^{r / 2}} x_{v}^{2 / 2}\right)^{1 / r}}{\left(\sum_{u=1}^{N} \sum_{v=1}^{N} c_{u, v} x_{u}^{1}{ }^{r / 2} x_{v}^{1}{ }^{r / 2}\right)^{1 / r}}$.

Then, we obtain $\quad \operatorname{QMII}_{r}\left(w^{0}, w^{2}, x^{0}, x^{2}\right)=Q M I I_{r}\left(w^{0}, w^{1}, x^{0}, x^{1}\right) \times$ $Q M I I_{r}\left(w^{1}, w^{2}, x^{1}, x^{2}\right)$. Therefore, $Q M I I_{r}$ satisfies T21. QED.

Proof of Corollary 3: First, we substitute (163) into the equation $\sigma^{2} / \sigma^{0}=\left(\sigma^{1} / \sigma^{0}\right) \times$ $\left(\sigma^{2} / \sigma^{1}\right)$. Then, we obtain $Q M P I_{r}\left(w^{0}, w^{2}, p^{0}, p^{2}, x^{0}, x^{2}, y^{0}, y^{2}\right)=$ $Q M P I_{r}\left(w^{0}, w^{1}, p^{0}, p^{1}, x^{0}, x^{1}, y^{0}, y^{1}\right) \times Q M P I_{r}\left(w^{1}, w^{2}, p^{1}, p^{2}, x^{1}, x^{2}, y^{1}, y^{2}\right)$

Therefore, $Q M P I_{r}$ satisfies T21. QED. 